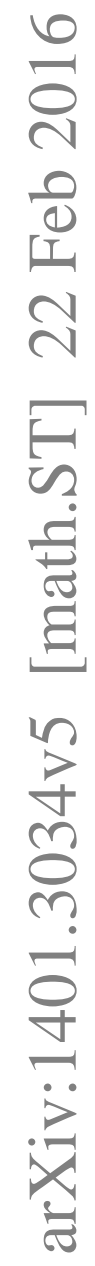




\title{
Inference for Monotone Functions Under Short and Long Range Dependence: Confidence Intervals and New Universal Limits
}

\author{
Pramita Bagchi, Moulinath Banerjee, and Stilian A. Stoev
}

July 2, 2018

\begin{abstract}
We introduce new point-wise confidence interval estimates for monotone functions observed with additive, dependent noise. Our methodology applies to both short- and long-range dependence regimes for the errors. The interval estimates are obtained via the method of inversion of certain discrepancy statistics. This approach avoids the estimation of nuisance parameters such as the derivative of the unknown function, which previous methods are forced to deal with. The resulting estimates are therefore more accurate, stable, and widely applicable in practice under minimal assumptions on the trend and error structure. The dependence of the errors especially long-range dependence leads to new phenomena, where new universal limits based on convex minorant functionals of drifted fractional Brownian motion emerge. Some extensions to uniform confidence bands are also developed.
\end{abstract}

Keywords. Isotonic Regression, Trend Estimation, Weak Dependence, Strong Dependence.

\section{Introduction}

The estimation of a trend function observed with additive noise is a canonical problem of substantial interest, widely studied in the statistical literature (see e.g. Clifford et. al.(2005)[9], Fan and Yao (2003)[11], Robinson (2009)[35], $\mathrm{Wu}$ and Zhao (2007) [46]). Most existing methods are based upon smoothness conditions on the trend (e.g. higher order differentiability, or curvature). They

*Pramita Bagchi is Postdoctoral Researcher, Department of Statistics, Ruhr University Bochum, 44801, Bochum, Germany (Email: Pramita.Bagchi@rub.de). Moulinath Banerjee is Professor, Department of Statistics, University of Michigan, Ann Arbor, MI 48109, USA (Email: moulib@umich.edu). Stilian Stoev is Associate Professor, Department of Statistics, University of Michigan, Ann Arbor, MI 48109, USA (Email: sstoev@umich.edu). The work of Banerjee was supported by NSF grant DMS-1308890 and a Sokol Faculty Award from University of Michigan. The work of Stoev was partially supported by NSF grant DMS1462368. The authors gratefully acknowledge the editor, associate editor and the referees for their helpful comments and suggestions 
do not incorporate shape constraints like monotonicity or convexity, even in the presence of such information. Monotonicity, in particular, is naturally associated with trend functions arising in many disciplines like climatology (e.g. global warming), environmental and air pollution (e.g. ground-level Ozone or fine particulate matter $\left(\mathrm{PM}_{10}\right.$ or $\left.\mathrm{PM}_{2.5}\right)$ as a function of temperature or humidity), engineering (diurnal trends in network traffic loads), among many others. One motivating application, illustrated in Figures 3 and 4 below, involves the annual global temperature anomalies data available at the NASA website [27]. The data comprises annual temperature records, measured relative to a baseline mean temperature, during the period 1850-1999; see also Jones and Mann (2002)[20] for a study of the paleoclimatic temperature and Steig et. al. (2009)[38] for evidence of warming trends at Antarctic locations. In the context of environmental pollution, monotone trends have been observed, for example, in the monitoring of water quality (Meal, 2001[22]), and mercury concentration of edible fish (Hussian et. al. 2005 [18]). In air pollution monitoring it is often the case that important factors (temperature, humidity, elevation) have an isotonic effect on the concentration of pollutants[26]. In many such scenarios, a natural model for the response as a function of time (or another natural covariate) is to write it as in (1) as the sum of an unobserved monotone trend function and dependent noise. A fundamental problem of interest is then to provide accurate confidence intervals for the underlying parameter that work well in practice under a variety of trend and dependence conditions on the data. It is desirable, for purposes of improved inference, to let the monotonicity constraint inform the statistical analysis of the data.

In the context of independent observations, the study of isotonic inference dates back to Rao (1969)[31]. Since then, the field has amassed a large body of research (see e.g. Banerjee and Wellner (2001, 2005)[4, 5], Banerjee $(2007,2009)$ [6, 7], Brunk (1970) [8], Groeneboom (1985) [13], Groeneboom and Wellner (1992) [15], Woodroofe and Sun (1993) [44], Sun and Woodroofe (1996) [40], to name a few). Yet, isotonic inference in the presence of dependence is relatively less developed, despite a clear need for it. Recent breakthrough was achieved thanks to the important work of Anevski and Hossjer (2006) [2] (henceforth AH) and Zhao and Woodroofe (2012)[47] (subsequently ZW). AH develops a general asymptotic scheme for inference under order restrictions that applies, in principle, to arbitrary dependence in the model. A number of practical, as well as, theoretical challenges, however, remain open. Most notably, deriving confidence intervals (based on the work $\mathrm{AH}$ and $\mathrm{ZW}$ ) requires estimation of the derivative $m^{\prime}\left(t_{0}\right)$ of the unknown function. This is known to be a difficult problem in the context of shape restricted inference and often leads to biased confidence intervals and substantial under-coverage in practice, as will be demonstrated later.

This paper develops new methodology (and the corresponding theory), purely within the isotonic regression framework, in the signal plus noise model for making inference on a monotone trend function that largely circumvents the nuisance parameter estimation problems above and is substantially more robust to functions ill-behaved around the point of interest. Our approach should be contrasted with ones that combine isotonization with smoothing; see, for example Mammen (1991) [21], Mukherjee (1988) [25], Pal and Woodroofe (2007) [28], 
Ramsay (1998) [30] where a variety of methods of this type have been developed in the i.i.d. framework, but typically under higher order smoothness assumptions. Here, our goal is to work under minimal smoothness assumptions and to provide estimates that apply to a broad variety of both weakly and strongly dependent (stationary) error structures.

In Section 5, we describe a general methodology for constructing point-wise confidence intervals in both short- and long-range dependence settings. The supporting theory is presented in Section 4. The performance of this methodology is studied with extensive simulations and shown to generally outperform existing estimates in terms of both coverage accuracy and length of the resulting intervals. Most notably, we offer a new type of dependence-adaptive procedure, which works under both weak and strong dependence in the data requiring minimal assumptions on the trend and dependence structure. We also extend our point-wise methodology to construct conservative confidence bands under shortrange dependence. Section 6 contains details of the simulation studies as well as applications of our methods to two real data problems where monotonicity constraints as well as dependence are natural and ubiquitous.

\section{Problem formulation and overview of the re- sults}

Consider the isotonic regression model

$$
Y_{i}=m\left(t_{i}\right)+\epsilon_{i}, \quad i=1, \cdots, n,
$$

where $m:[0,1] \rightarrow \mathbb{R}$ is an unknown monotone non-decreasing function, $t_{i}=$ $i / n, i=1, \cdots, n$ is a fixed uniform design and where the errors $\epsilon_{i}$ have zero means and variance $\operatorname{Var}\left(\epsilon_{i}\right)=\sigma^{2}$. We are interested in the case where the noise is dependent. We shall model it by a stationary time series $\left\{\epsilon_{k}\right\}_{k \in \mathbb{Z}}$, which may have either weak or strong dependence (cf Section 3, below). The trend $m$ will be assumed monotone non-decreasing and to satisfy the following mild condition.

Assumption C. The regression function $m(t)$ is continuously differentiable in a neighborhood of $t_{0}$ with $m^{\prime}\left(t_{0}\right)>0$.

Our ultimate goal is to construct an asymptotic confidence interval for $m\left(t_{0}\right)$, $\left(0<t_{0}<1\right)$, which is largely robust to the dependence structure of the errors. To this end, we consider the testing problem: $H_{0}: m\left(t_{0}\right)=\theta_{0}$ vs. $H_{1}: m\left(t_{0}\right) \neq$ $\theta_{0}$. Confidence intervals for $m\left(t_{0}\right)$ will be obtained by inversion of acceptance regions of tests for the above problem. Consider the usual isotonic regression estimate (IRE) of $m$ (cf. Robertson et. al. (1988)[34]), obtained as

$$
\left(\hat{m}_{n}\left(t_{i}\right), i=1, \cdots, n\right)=\underset{m_{1} \leq \cdots \leq m_{n}}{\operatorname{Argmin}} \sum_{i=1}^{n}\left(Y_{i}-m_{i}\right)^{2} .
$$

To address the above testing problem, we also consider the following constrained 
isotonic estimate $\hat{m}_{n}^{0}$. Let $l=\left\lfloor n t_{0}\right\rfloor$, so that $t_{l} \leq t_{0}<t_{l+1}$ and define

$$
\left(\hat{m}_{n}^{0}\left(t_{i}\right), i=1, \cdots, n\right)=\underset{m_{1} \leq \cdots \leq m_{l} \leq \theta_{0} \leq m_{l+1} \leq \cdots \leq m_{n}}{\operatorname{Argmin}} \sum_{i=1}^{n}\left(Y_{i}-m_{i}\right)^{2} .
$$

Note that both functions $\hat{m}_{n}$ and $\hat{m}_{n}^{0}$ are identified only at the grid points. By convention, we extend them as left-continuous piece-wise constant functions defined on the entire interval $(0,1]$.

Our hypothesis tests will be based on the following discrepancy statistics

$$
\begin{aligned}
& L_{n}=\frac{n}{\sigma_{n}^{2}}\left(\sum_{i=1}^{n}\left(Y_{i}-\hat{m}_{n}^{0}\left(t_{i}\right)\right)^{2}-\sum_{i=1}^{n}\left(Y_{i}-\hat{m}_{n}\left(t_{i}\right)\right)^{2}\right) \equiv \frac{n}{\sigma_{n}^{2}} \mathbb{L}_{n} \\
& T_{n}=\frac{n}{\sigma_{n}^{2}} \sum_{i=1}^{n}\left(\hat{m}_{n}\left(t_{i}\right)-\hat{m}_{n}^{0}\left(t_{i}\right)\right)^{2} \equiv \frac{n}{\sigma_{n}^{2}} \mathbb{T}_{n},
\end{aligned}
$$

where $\sigma_{n}^{2}=\operatorname{Var}\left(\sum_{k=1}^{n} \epsilon_{k}\right)$. A third statistic which will prove particularly useful in the long-range dependence case is the 'ratio statistic' $R_{n}:=L_{n} / T_{n}$. Its asymptotic properties will be derived from the joint asymptotic behavior of $L_{n}$ and $T_{n}$.

In the rest of this section, we give the intuition behind our methodology. The precise asymptotic results are given in Section 4. Consider the rescaled discrepancy statistics $\mathbb{L}_{n}$ and $\mathbb{T}_{n}$ in (4). As shown in Banerjee (2009)[7], in the i.i.d. setting if $m\left(t_{0}\right)=\theta_{0}$, then $\mathbb{L}_{n} / \sigma^{2} \Rightarrow \mathbb{L}$, where $\mathbb{L}$ is a positive random variable expressed as a functional of the two-sided Brownian motion plus quadratic drift $\left\{\mathbb{W}(t)+t^{2}\right\}_{t \in \mathbb{R}}$ and $\sigma^{2}$ is the common variance of the errors. Further, the closely related $L^{2}$-type discrepancy statistic $\mathbb{T}_{n}$ is similarly shown to satisfy: $\mathbb{T}_{n} / \sigma^{2} \Rightarrow \mathbb{T}$, where the limit $\mathbb{T}$ is another functional of $\left\{\mathbb{W}(t)+t^{2}\right\}_{t \in \mathbb{R}}$. As both these limits are universal (free of the parameters of the problem), the confidence sets obtained by the method of inversion of these statistics do not involve the nuisance parameter $m^{\prime}\left(t_{0}\right)$ - a most challenging quantity to estimate in practice. Only an estimate of $\sigma^{2}$ is required, which easy to obtain in practice. This methodology in the i.i.d. setting has lead to accurate confidence intervals, which work well in practice for rather challenging isotonic trends. The asymptotic phenomena encountered in the i.i.d. case then naturally motivate us to investigate whether similar benefits accrue from the above statistics in the dependent case.

In the case of dependent errors two fundamentally different regimes arise: $(i)$ short-range dependence and (ii) long-range dependence. The finite variance stationary time series in (1) is said to be long-range dependent if $\sum_{k=1}^{\infty}\left|\operatorname{Cov}\left(\epsilon_{k}, \epsilon_{0}\right)\right|=$ $\infty$ and if the latter covariances are summable, it is referred to as short-range dependent (see e.g. Doukhan et. al. (2003) [10]). We refer to $\operatorname{Cov}\left(\epsilon_{k}, \epsilon_{0}\right)$ as $\operatorname{Cov}(k)$, the covariance at lag $k$ which is well-defined by stationarity.

In the short-range dependence context, one expects that the discrepancy statistics $\mathbb{L}_{n}$ and $\mathbb{T}_{n}$ will have similar asymptotic behavior to the independent errors situation. We show in Theorem 4.1 below that, under mild assumptions, this is indeed the case, and in fact, under $m\left(t_{0}\right)=\theta_{0}$, we have the following 
joint convergence:

$$
\left(\mathbb{L}_{n}\left(\theta_{0}\right) / \tau^{2}, \mathbb{T}_{n}\left(\theta_{0}\right) / \tau^{2}\right) \Longrightarrow(\mathbb{L}, \mathbb{T}), \quad \text { as } n \rightarrow \infty,
$$

where $\tau^{2}=\sum_{k=-\infty}^{\infty} \operatorname{Cov}(k)$ and $\mathbb{L}$ and $\mathbb{T}$ are functionals of the same quadratically drifted standard Brownian motion. In practice, this result justifies an effective and robust inference methodology for constructing confidence intervals for $m\left(t_{0}\right)$ via the method of inversion (Section 5.2), where the only parameter that needs to be estimated is $\tau^{2}$.

In the long-range dependence setting, the discrepancy statistics $\mathbb{L}_{n}$ and $\mathbb{T}_{n}$ exhibit fundamentally new behavior. In fact, the problem here is ill-posed since multiple different types of long-range dependence may arise (Samorodnitsky, 2006) [37]. In this paper, we focus on one of the most frequently encountered long-range dependence regimes where the cumulative sums of the error sequence converge to the fractional Brownian motion (fBm, in short): see Section 3. In this setting, from Theorem 4.2 below, we get that

$$
r_{n}\left(\mathbb{L}_{n}\left(\theta_{0}\right), \mathbb{T}_{n}\left(\theta_{0}\right)\right) \Longrightarrow C\left(\mathbb{L}^{(H)}, \mathbb{T}^{(H)}\right),
$$

where the factor $r_{n}$ and the constant $C$ are both unknown and the limits $\mathbb{L}^{(H)}$ and $\mathbb{T}^{(H)}$ are now expressed as functionals of a standard two-sided fBm plus quadratic drift $\left\{B_{H}(t)+t^{2}\right\}_{t \in \mathbb{R}}$. The functionals defining $\mathbb{L}^{(H)}$ and $\mathbb{T}^{(H)}$ are similar to those defining $\mathbb{L}$ and $\mathbb{T}$, respectively. Their analysis, however, requires new results on the behavior of greatest convex minorants of $\mathrm{fBm}$ plus quadratic drift, which may be of independent interest (see Supplement B). The distributions of $\mathbb{L}^{(H)}$ and $\mathbb{T}^{(H)}$ only depend on the Hurst parameter $H \in(1 / 2,1)$ of the fractional Brownian motion.

In contrast to the weak dependence case, inversion of the $\mathbb{L}_{n}$ or $\mathbb{T}_{n}$ statistics to construct confidence intervals in the long-range dependence case requires the estimation of $m^{\prime}\left(t_{0}\right)$, since the constant $C$ depends on it. One can eliminate this nuisance parameter, however, by considering the ratio statistic $R_{n}:=\mathbb{L}_{n} / \mathbb{T}_{n}$. This can be thought of as a self-normalization. We show that the limit distribution of $R_{n}$ depends only on the Hurst parameter $H$ (Theorem 5.1). This result, again through the method of inversion, yields confidence intervals for $m\left(t_{0}\right)$ without having to estimate the derivative. In practice, $H$ is the only parameter that needs to be estimated, but this problem has received considerable attention in the literature (see e.g. Fay et. al. (2009)[12] and references therein). Our confidence intervals based on plug-in estimates of $H$ have close to nominal coverage (cf Table 6 in Supplement A). In fact, a certain partial nesting property of the quantiles of the asymptotic distribution can be used to obtain conservative confidence intervals without having to estimate $H$ precisely (see, Section 6 , below for more detials). Furthermore, the new ratio statistic can also be used in the short-range dependence and, in particular, the i.i.d. context $(H=1 / 2)$. It provides an alternative methodology to that based on the discrepancy statistics $\mathbb{L}_{n}$ and $\mathbb{T}_{n}$, where in fact no estimation of $\tau^{2}$ is necessary!

Selected quantiles of this new family of universal limit distributions are tabulated in the short- and long-range dependence regimes (Table 1 in the paper and Tables 1 through 4 in Supplement B). A striking feature of these quantiles, discussed in detail in Section 6.1, is a type of partial nesting as a function of $H$. 
It allows one to obtain conservative intervals with $90 \%$ or $95 \%$ nominal coverage from the ratio statistic without a precise estimate of $H$, provided the true value of $H$ is not very close to 1 . Thus, inference based on the ratio statistic provides a remarkable degree of robustness across both short and long-range dependence.

\section{Dependence structure}

In this section, we introduce and discuss our formal assumptions on the dependence structure of the errors $\epsilon_{i}$ 's in (1). These assumptions will be tacitly adopted for the rest of the paper and will require some technicalities for a precise description.

We suppose that the errors have zero means, finite variances and form a strictly stationary time series $\left\{\epsilon_{k}\right\}_{k \in \mathbb{Z}}$. Let $S_{n}=\sum_{k=1}^{n} \epsilon_{k}$, and consider the piece-wise linear cumulative sum diagram

$$
w_{n}(t)=\frac{1}{\sigma_{n}}\left(\sum_{i=1}^{\lfloor n t\rfloor} \epsilon_{i}+(n t-\lfloor n t\rfloor) \epsilon_{\lfloor n t\rfloor+1}\right), \quad \text { where } \quad \sigma_{n}^{2}=\operatorname{Var}\left(S_{n}\right) .
$$

The asymptotic behavior of the process $\left\{w_{n}(t)\right\}_{t \geq 0}$ is generally determined by the degree of dependence of the errors in addition to their tail behavior. If the $\epsilon_{i}$ 's are weakly dependent, then as in the usual Donsker theorem, the limit is the Brownian motion and the corresponding statistical results are similar to the situation of independent errors. On the other hand, as noted in the introduction, strong dependence of the $\epsilon_{i}$ 's leads to different types of limits and new statistical theory. We shall consider two different regimes: [i] short-range dependent errors, and, [ii] long-range dependent errors, as explained in Section II.

\subsubsection{Short-range dependence}

To formalize weak dependence, let $\|\cdot\|$ denote the $L^{2}$ norm on the probability space and introduce the discrete filtration $\mathcal{F}_{n}=\sigma\left\{\epsilon_{m}, m \leq n\right\}, n \in \mathbb{Z}$, i.e. $\mathcal{F}_{n}$

is the $\sigma$-algebra generated by all errors up to and including 'time' $n$. In the short-range dependent case, following ZW[47], we assume that

$$
\sum_{n=1}^{\infty} n^{-\frac{3}{2}}\left\|\mathbb{E}\left(S_{n} \mid \mathcal{F}_{0}\right)\right\|<\infty
$$

It is shown in Peligrad and Utev (2005)[29] that if (6) is satisfied then,

$$
\Gamma:=\sum_{k=0}^{\infty} 2^{-\frac{1}{2} k}\left\|\mathbb{E}\left(S_{2^{k}} \mid \mathcal{F}_{0}\right)\right\|<\infty \quad \text { and } \quad \mathbb{E}\left[\max _{k \leq n} S_{k}^{2}\right] \leq 6\left[\mathbb{E}\left(\epsilon_{1}^{2}\right)+\Gamma\right] n .
$$

Furthermore, the limit

$$
\tau^{2}=\lim _{n \rightarrow \infty} \frac{1}{n} \mathbb{E}\left(S_{n}^{2}\right)<\infty
$$


exists and the process $\left\{w_{n}(t)\right\}_{0 \leq t \leq 1}$ converges in distribution to the Brownian motion $\mathbb{B}$ in the space $D[0,1]$ equipped with the usual $J_{1}$-Skorohod topology.

Remark 3.1. In AH[2], weak dependence was quantified in terms of mixing conditions. Here, we use an alternative condition (6) from ZW[47], implied by the strong mixing Assumption (A9) of AH[2], and therefore weaker.

\subsubsection{Long-range dependence}

A great variety of models exhibit long-range dependence. We focus here on a special but important case when the error $\epsilon_{k}=g\left(\xi_{k}\right), k \in \mathbb{Z}$, where $\left\{\xi_{i}\right\}_{i \in \mathbb{Z}}$ is a stationary Gaussian time series with zero mean. The function $g$ is deterministic and from $L^{2}(\phi)$ where $\phi$ denotes standard normal density, i.e., $\mathbb{E}(g(Z))^{2}=$ $E\left(\epsilon_{1}^{2}\right)<\infty$ where $Z \sim N(0,1)$. In this setting, an elegant theory characterizing the possible limits of the cumulative sums in (5) was developed in the seminal work of Taqqu (1975[41], 1979[42]).

Following $\mathrm{AH}[2]$, let $\operatorname{Cov}(k)=\mathbb{E}\left(\xi_{i} \xi_{i+k}\right)$ be such that $\operatorname{Cov}(0)=1$ and $\operatorname{Cov}(k)=k^{-d} l_{0}(k)$, where $0<d<1$ is fixed and $l_{0}$ is a function slowly varying at infinity, i.e., for all $a>0, l_{0}(a x) / l_{0}(x) \rightarrow 1$, as $x \rightarrow \infty$.

As $\mathbb{E}\left(g(Z)^{2}\right)<\infty$, the function $g$ can be expanded using Hermite polynomials and we have the representation:

$$
\epsilon_{i}:=g\left(\xi_{i}\right)=\sum_{k=r}^{\infty} \frac{\eta_{k}}{k !} H_{k}\left(\xi_{i}\right),
$$

where the series converges in $L^{2}(\mathbb{P}), \eta_{k}=\mathbb{E}\left(g\left(\xi_{i}\right) H_{k}\left(\xi_{i}\right)\right), k \geq r$; the $H_{k}$ 's are the Hermite polynomials of order $k$ and the summation starts from $r \geq 1-$ the index of the first nonzero coefficient in the expansion. Note that $r \geq 1$ corresponds to centered $\epsilon_{i}$ 's, since $E\left(H_{k}\left(\xi_{1}\right)\right)=0$ for all $k \geq 1$. The index $r$ is referred to as the Hermite rank of the function $g$. For the rest of the paper we restrict our discussion to the case $r=1$

The results of Taqqu (1975[41], 1979[42]) show that if $0<d<1$, the sequence $\left\{\epsilon_{i}\right\}$ also exhibits long-range dependence and, in fact,

$$
\left\{\sigma_{n}^{-1} w_{n}(t)\right\}_{t \in[0,1]} \Longrightarrow\left\{B_{H}(t)\right\}_{t \in[0,1]},
$$

in $D[0,1]$ equipped with Skorohod topology, where the limit process $B_{H}$ is a Gaussian process in $C[0,1]$ a.s. with stationary increments. It can be shown that

$$
\sigma_{n}^{2}=\eta_{1}^{2} n^{2-d} l_{1}(n)(1+o(1))
$$

where $l_{1}$ is another slowly varying function: $\left.l_{1}(k)=2 l_{0}(k) /(1-d)(2-d)\right)$. The process $B_{H}$ can be uniquely extended to a process on the entire line with stationary increments which is denoted by the same symbol and known as the fractional Brownian motion (fBm) with self-similarity parameter (also called Hurst index) $H=1-d / 2$ : i.e. for all $c>0$, the processes $\left\{B_{H}(c t)\right\}_{t \in \mathbb{R}}$ and $\left\{c^{H} B_{H}(t)\right\}_{t \in \mathbb{R}}$ are equal in distribution. The stationarity of the increments and 
self-similarity imply that

$$
\operatorname{Cov}\left(B_{H}(t), B_{H}(s)\right)=\frac{\sigma^{2}}{2}\left(|t|^{2 H}+|s|^{2 H}-|t-s|^{2 H}\right), \quad t, s \in \mathbb{R} .
$$

For more details on the properties of the $\mathrm{fBm}$, see e.g. the review chapter by Taqqu in Doukhan et. al. (2003)[10].

\section{Key results}

We present in this section the joint asymptotic behavior of the statistics $L_{n}$ and $T_{n}$ which is central to the subsequent methodology. To this end, we first introduce the greatest convex minorant (GCM) functional.

Greatest convex minorants: Let $\mathcal{T}_{I}(f)$ denote the GCM of a real-valued function $f$, defined on an interval $I \subseteq \mathbb{R}$. For an interval $J \subset I$, we denote the GCM of the restriction of $f$ to $J$ by $\mathcal{T}_{J}(f)$. When $f$ is defined on $\mathbb{R}$, we sometimes write $\mathcal{T}(f)$ for $\mathcal{T}_{\mathbb{R}}(f)$ and $\mathcal{T}_{c}(f)$ for $\mathcal{T}_{[-c, c]}(f)$. Also, let $\mathcal{L}(f)$ denote the left derivative functional of a convex function $f$, which is a well-defined, non-decreasing and left-continuous function (cf. Theorem 24.1 of Rockafellar (1970)[36]).

Define the process: $\{\mathbb{G}(z)\}_{z \in \mathbb{R}} \equiv\left\{\mathbb{G}_{a, b}(z)\right\}_{z \in \mathbb{R}}:=\left\{a \mathbb{W}(z)+b z^{2}\right\}_{z \in \mathbb{R}}$, where $b=\frac{1}{2} m^{\prime}\left(t_{0}\right)$ and (i) (under weak dependence) $\mathbb{W}$ is a two-sided Brownian motion on $\mathbb{R}$, and $a:=\tau$ given in (8), (ii) (under strong dependence) $\mathbb{W}$ is the fBm process $B_{H}$ and $a:=\left|\eta_{1}\right|$.

Next, define the following slope-of-greatest-convex-minorant functionals as follows:

$$
\begin{aligned}
& \mathcal{S}_{a, b}(z)=\mathcal{L} \circ \mathcal{T}(\mathbb{G})(z) \\
& \mathcal{S}_{a, b}^{h}(z)= \begin{cases}\mathcal{L} \circ \mathcal{T}_{(-\infty, 0)}(\mathbb{G})(z) \wedge h & , z \in(-\infty, 0) \\
\lim _{u \uparrow 0} \mathcal{L} \circ \mathcal{T}_{(-\infty, 0)}(\mathbb{G})(u) \wedge h & , z=0 \\
\mathcal{L} \circ \mathcal{T}_{(0, \infty)}(\mathbb{G})(z) \vee h & , z \in(0, \infty)\end{cases}
\end{aligned}
$$

We are now ready to state the limit distributions of the statistics $L_{n}$ and $T_{n}$ in terms of $\mathcal{S}_{a, b}(z)$ and $\mathcal{S}_{a, b}^{0}(z)$. Define:

$$
\mathbb{L}_{a, b}=\int_{\mathbb{R}}\left(\left(\mathcal{S}_{a, b}(z)\right)^{2}-\left(\mathcal{S}_{a, b}^{0}(z)\right)^{2}\right) d z \text { and } \mathbb{T}_{a, b}=\int_{\mathbb{R}}\left(\mathcal{S}_{a, b}(z)-\mathcal{S}_{a, b}^{0}(z)\right)^{2} d z .
$$

Remark 4.1. The processes $\mathcal{S}_{a, b}(z)$ and $\mathcal{S}_{a, b}^{0}(z)$ differ on a compact interval. This is rigorously established in Theorem 3.1 of Supplement $B$ showing that the statistics in (13) are proper random variables.

Remark 4.2. In the long-range dependence case where $\mathbb{L}_{a, b}$ and $\mathbb{T}_{a, b}$ depend on the Hurst index $H$, we denote them $b y \mathbb{L}_{a, b}^{(H)}$ and $\mathbb{T}_{a, b}^{(H)}$. When $a=b=1$, we drop the subscripts and write $\mathbb{L}$ and $\mathbb{T}$ in the short-range dependence case, and $\mathbb{L}^{(H)}$ and $\mathbb{T}^{(H)}$ in the long-range dependence case. In the following sections 
we will, often, drop $H$ and just use $\mathbb{L}$ and $\mathbb{T}$ for both short- and long-range dependence when there is no chance of confusion.

Theorem 4.1. For short-range dependent errors, $\left(L_{n}, T_{n}\right) \Rightarrow(\mathbb{L}, \mathbb{T})$, as $n \rightarrow \infty$.

Remark 4.3. Since $\sigma_{n}^{2} \sim n \tau^{2}$ under short-range dependence, the above result can be rewritten as:

$$
\frac{1}{\tau^{2}}\left(\mathbb{L}_{n}, \mathbb{T}_{n}\right) \Rightarrow(\mathbb{L}, \mathbb{T}) \text {, as } n \rightarrow \infty .
$$

Theorem 4.2. For long-range dependent errors, as $n \rightarrow \infty$,

$$
\frac{\sigma_{n}^{2}}{n^{2} d_{n}^{3}}\left(L_{n}, T_{n}\right) \Longrightarrow a^{2}\left(\frac{a}{b}\right)^{\frac{2 H-1}{2-H}}\left(\mathbb{L}^{(H)}, \mathbb{T}^{(H)}\right),
$$

where $a=\left|\eta_{1}\right|, b=\frac{1}{2} m^{\prime}\left(t_{0}\right), \sigma_{n}^{2}$ is as defined at the beginning of Section III and $d_{n} \rightarrow 0$ is as in Theorem A.1 (ii).

The proof in the long-range dependent case is given in Appendix B. The proof in the simpler, short-range case is similar and omitted for brevity. For a proof-sketch of the asymptotics of $L_{n}$ and $T_{n}$ see Appendix A.

Remark 4.4. Using the definitions of $L_{n}, T_{n}$ in (4), the result of Theorem 4.2 can be restated as:

$$
\frac{1}{n d_{n}^{3}}\left(\mathbb{L}_{n}, \mathbb{T}_{n}\right) \Longrightarrow a^{2}\left(\frac{a}{b}\right)^{\frac{2 H-1}{2-H}}\left(\mathbb{L}^{(H)}, \mathbb{T}^{(H)}\right) .
$$

Now, by Theorem $A .1$ (ii), $d_{n}=n^{-d /(2+d)} l_{2}(n)$ where $d$ is the long memory parameter encountered in Section III.2 and $l_{2}(n)$ is a slowly varying function related to $l_{1}(n)$ that appears in the representation of $\sigma_{n}^{2}$ in (10). The long-range dependent error structures generally used in statistical applications, and, in particular, in our paper - namely, Fractional Gaussian Noise (FGN) and FARIMA processes - have trivial slowly varying functions: $l_{1}(n)=1$ and (therefore) $l_{2}(n)=1$, and in such cases the above display further simplifies to:

$$
\begin{aligned}
\frac{1}{n^{1-3 d /(2+d)}}\left(\mathbb{L}_{n}, \mathbb{T}_{n}\right) & =n^{-(2 H-1) /(2-H)}\left(\mathbb{L}_{n}, \mathbb{T}_{n}\right) \\
& \Longrightarrow a^{2}\left(\frac{a}{b}\right)^{\frac{2 H-1}{2-H}}\left(\mathbb{L}^{(H)}, \mathbb{T}^{(H)}\right),
\end{aligned}
$$

using $H=1-d / 2$.

\section{Methodology}

\subsection{An asymptotically pivotal ratio statistic}

Recall (14). To be able to use the statistics $\mathbb{L}_{n}, \mathbb{T}_{n}$, one needs a suitable 'plug-in' estimate for $\tau^{2}$ in (8), but this can typically be estimated well in 
practice. The use of the statistics $\mathbb{L}_{n}$ and $\mathbb{T}_{n}$ in the LRD case, however, is more problematic because by (17), the limit distributions involve the derivative $m^{\prime}\left(t_{0}\right)$ (appearing in the constant $b$ ) which is difficult to estimate, and in addition, the quantities $\eta_{1}$ (appearing in the constant $a$ ) and $H$ (both in the normalization of the test statistics and the limit distributions). An elegant way to eliminate the need for estimating $m^{\prime}\left(t_{0}\right)$ as well as $\eta$ and the $H$-dependent normalization on the right side of (17) is to consider the ratio statistic, introduced next.

Note that $L_{n}$ and $T_{n}$ are always non-negative by definition. By (4), if $T_{n}=0$ we have $L_{n}=0$. Also, as shown in Lemma 3.1 from Supplement A, we have $L_{n} \geq T_{n}$. Therefore $L_{n}$ and $T_{n}$ are either both equal to 0 or both strictly positive. Similarly (13) implies that if $\mathbb{T}=0$, then $\mathbb{L}=0$ and by Theorems 4.1 and 4.2 and the Portmanteau theorem, we obtain that $\mathbb{L} \geq \mathbb{T}$ almost surely. It follows again that $\mathbb{T}$ and $\mathbb{L}$ are also either both equal to 0 or strictly positive. Now, define the ratio statistic $R_{n}=L_{n} / T_{n} \equiv \mathbb{L}_{n} / \mathbb{T}_{n}$, where $0 / 0$ is interpreted as 1 . By the discussion in the above paragraph, $\mathbb{P}\left(R_{n}<\infty\right)=1$.

Theorem 5.1. For both short- and long-range dependent errors, we have

$$
R_{n} \Longrightarrow \mathcal{R}:=\frac{\mathbb{L}}{\mathbb{T}}, \quad \text { as } n \rightarrow \infty,
$$

where the limit has a proper probability distribution.

Proof. The convergence follows from Theorems 4.1, 4.2 and the Continuous Mapping Theorem, provided that $\mathbb{P}(\mathbb{T}=0)=0$. The latter is true thanks to Theorem 3.2 in Supplement B.

Remark 5.1. Note that though the computation of $R_{n}$ does not require us to know/estimate $H$, the limit distribution of $R_{n}$ does involve the Hurst parameter.

\subsection{Construction of Confidence Intervals}

We first focus on the use of the statistics $\mathbb{L}_{n}$ and $\mathbb{T}_{n}$.

Short-range dependence: Let $\mathbb{L}_{n}(\theta)$ and $\mathbb{T}_{n}(\theta)$ denote the residual sum of squares and $L_{2}$ statistics, respectively, for testing $H_{0}: m\left(t_{0}\right)=\theta$ against $H_{a}: m\left(t_{0}\right) \neq \theta$. Letting $\theta_{0}$ denote the true value of $m\left(t_{0}\right)$, an asymptotic level $1-\alpha$ confidence set for $\theta_{0}$, using inversion of $L_{n}$, is given by

$\left\{\theta: \mathbb{L}_{n}(\theta) / \hat{\tau}^{2} \leq F_{\mathbb{L}}^{\leftarrow}(1-\alpha)\right\}$, where $F_{\mathbb{L}}^{\leftarrow}$ denotes the left-continuous quantile function of $F_{\mathbb{L}}$, the distribution function of $\mathbb{L}$ and $\hat{\tau}$ is a consistent estimate of $\tau$. For weakly dependent errors $\tau^{2}$ was estimated as

$$
\hat{\tau}^{2}=\hat{\gamma}_{n}(0)+2 \sum_{k \leq \sqrt{n}}\left(1-\frac{k}{\sqrt{n}}\right) \hat{\gamma}_{n}(k)
$$

where $\hat{\gamma}_{n}(k)$ is the empirical auto-covariance of $\hat{e}_{i}:=Y_{i}-\hat{m}_{n}\left(t_{i}\right)$ at lag $k$, i.e. $\hat{\gamma}_{n}(k)=n^{-1} \sum_{i=1}^{n-k} \hat{e}_{i} \hat{e}_{i+k}$. This is a consistent estimator under the presence of a monotone trend as shown in Theorem 2 of Wu et. al. (2001)[45]. 
The statistic $T_{n}$ can be used in an exact similar fashion to obtain confidence intervals.

By our results on the shape of $L_{n}(\theta)$ and $T_{n}(\theta)$ (which completely describe the shapes of $\mathbb{L}_{n}(\theta)$ and $\left.\mathbb{T}_{n}(\theta)\right)$ in Lemma 3.2 of Supplement A, letting,

$C_{L}(\alpha):=\inf \left\{\theta: \mathbb{L}_{n}(\theta) / \hat{\tau}^{2}<F_{\mathbb{L}}^{\leftarrow}(1-\alpha)\right\}$ and $C_{U}(\alpha):=\sup \left\{\theta: \mathbb{L}_{n}(\theta) / \hat{\tau}^{2}<F_{\mathbb{L}}^{\leftarrow}(1-\alpha)\right\}$,

we conclude that $\left[C_{L}(\alpha), C_{U}(\alpha)\right]$ is precisely the set $\left\{\theta: L_{n}(\theta) \leq F_{\mathbb{L}}^{\leftarrow}(1-\alpha)\right\}$, giving us an asymptotic $100(1-\alpha) \%$ confidence interval for $\theta_{0}$. The lemma also ensures that the intervals produced by inverting $\mathbb{L}_{n}$ and $\mathbb{T}_{n}$ are always of finite length.

Long-range dependence: If $\hat{H}$ is a polynomial-rate-consistent estimate of $H$ : i.e. $n^{\lambda}(\hat{H}-H)=O_{p}(1)$ for some $\lambda>0$, then

$$
n^{-(2 \hat{H}-1) /(2-\hat{H})}\left(\mathbb{L}_{n}, \mathbb{T}_{n}\right) \Longrightarrow a^{2}\left(\frac{a}{b}\right)^{\frac{2 H-1}{2-H}}\left(\mathbb{L}^{(H)}, \mathbb{T}^{(H)}\right) .
$$

The above result follows immediately from (17) and the fact that for a polynomial-rate-consistent $\hat{H}, n^{-(2 \hat{H}-1) /(2-\hat{H})+(2 H-1) /(2-H)} \rightarrow_{p} 1$.

Furthermore, if $\hat{a}, \hat{b}$ are consistent estimates of $a, b$ and $F_{\mathbb{L}^{(H)}}^{\leftarrow}(1-\alpha)$, the $(1-\alpha)^{\prime}$ th quantile of $\mathbb{L}^{(H)}$ is continuous in $H$, as suggested strongly by extensive simulations,

$\left\{\theta: n^{-(2 \hat{H}-1) /(2-\hat{H})} \mathbb{L}_{n}(\theta) \leq \hat{a}^{2}(\hat{a} / \hat{b})^{(2 \hat{H}-1) /(2-\hat{H})} F_{\mathbb{L}^{(H)}}^{\overleftarrow{ }(1-\alpha)\}}\right.$ gives an asymptotic level $1-\alpha$ confidence set for $\theta_{0}$, by an easy application of Slutsky's lemma. As under short-range dependence, this is a confidence interval. Details on the estimation of $H$ for our simulations and data analysis are provided later. Simulated quantiles for $\mathbb{L}$ for both short- and long-range dependent errors with different Hurst parameters $H$ are presented in Tables 3 and 4 , Supplement B. In the sequel we will often refer to the $\mathbb{L}_{n}$ based intervals as $L_{n}$ based intervals.

We next turn to confidence sets using $R_{n}$ which avoids the estimation of difficult nuisance parameters in the long-range dependence case as discussed previously. Consider first, the shape of $R_{n}(\theta)$ as a function of $\theta$. It assumes the value 1 at $\theta=\hat{m}_{n}\left(t_{0}\right)$, converges to 1 as $|\theta| \rightarrow \infty$ and displays irregular humps in between. Figure 1 illustrates the behavior of this statistic as a function of $h$, where $h=n^{1 / 3}\left(\theta-\theta_{0}\right)$ under SRD and $h=d_{n}^{-1}\left(\theta-\theta_{0}\right)$ under LRD. As a sensible inversion of $R_{n}$ should avoid values away from $\hat{m}_{n}\left(t_{0}\right)$, an asymptotic confidence set should look like: $\left\{\theta: R_{n}(\theta)>\zeta\right\}$, where $\zeta>1$ is an appropriate quantile (depending on the level of confidence desired) of $\mathcal{R}$, the limiting random variable in Theorem 5.1. This will, however, not yield a confidence interval but a rather irregular confidence set, and, in particular, may miss values of $\theta$ close to $\theta_{0}$.

Another issue with using $R_{n}$ is that the quantiles of $\mathcal{R}$ grow extremely slowly from 1 and are hard to represent in a table. For matters of practical 


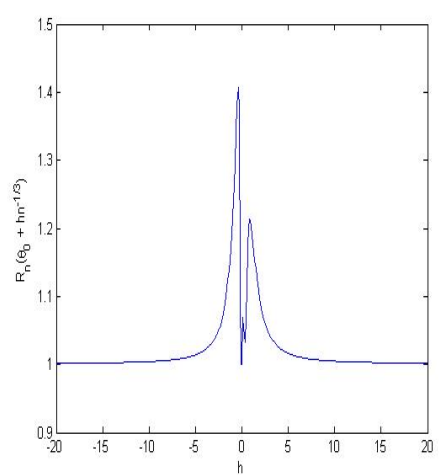

(a) Short-range dependence

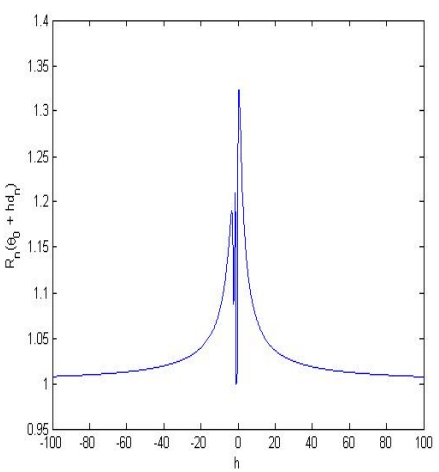

(b) Long-range dependence

Figure 1: Shape of ratio statistic as a function of $h$

convenience, we therefore make a monotone transformation of $R_{n}$, namely,

$$
\Psi_{n}(\theta)= \begin{cases}-\log \left(R_{n}(\theta)-1\right), & \text { if } R_{n}(\theta)>1 \\ \infty, & \text { if } R_{n}(\theta)=1\end{cases}
$$

Then, the following Proposition follows easily from Theorem 5.1 and the Continuous Mapping Theorem.

Proposition 5.1. Under the assumptions of Theorem 5.1, we have

$$
\Psi_{n}\left(\theta_{0}\right) \stackrel{d}{\rightarrow} \Psi:=-\log (\mathcal{R}-1) \text { as } n \rightarrow \infty .
$$

where $\mathbb{P}(\Psi=\infty)=\mathbb{P}(\mathcal{R}=1)$.

As $\Psi_{n}$ is a monotone decreasing transformation of $R_{n}=L_{n} / T_{n}$, it exhibits the same irregularities; see Figure 1 in Supplement A, and therefore, in terms of $\Psi_{n}$, our confidence set $\left\{\theta: \Psi_{n}(\theta)<-\log (\zeta-1)\right\}$, is still irregular. To avoid this, we propose a confidence interval of the form $\left[\widetilde{C}_{L}(\alpha), \widetilde{C}_{u}(\alpha)\right]$, where $\widetilde{C}_{L}$ and $\widetilde{C}_{U}$ are defined thus:

$\widetilde{C}_{L}(\alpha):=\inf \left\{\theta: \Psi_{n}(\theta)<F_{\Psi}^{\leftarrow}(1-\alpha)\right\}, \quad \widetilde{C}_{U}(\alpha):=\sup \left\{\theta: \Psi_{n}(\theta)<F_{\Psi}^{\overleftarrow{ }}(1-\alpha)\right\}$

Note that this gives us a conservative $100(1-\alpha) \%$ C.I. for $\theta_{0}$. Selected quantiles of $\Psi$ are presented in Table 1I. See, Supplement B, Tables 1 and 2 for a detailed presentation of the quantiles of $\Psi$.

While our knowledge of the behavior of $R_{n}(\theta)$ is limited, we do have the following result.

Proposition 5.2. Let $\theta \neq \theta_{0}$ and $R_{n}(\theta)$ be the ratio statistic calculated under the null hypothesis $H_{0, \theta}: m\left(t_{0}\right)=\theta$. Then, $R_{n}(\theta) \stackrel{P}{\rightarrow} 1$ as $n \rightarrow \infty$. 
Table 1: Quantiles of $\Psi$

\begin{tabular}{|c|c|c|c|c|c|}
\hline $\mathrm{p}$ & $\mathrm{SRD}$ & $\mathrm{H}=0.7$ & $\mathrm{H}=0.8$ & $\mathrm{H}=0.9$ & $\mathrm{H}=0.95$ \\
\hline 0.50 & $2.21(0.021)$ & $2.19(0.006)$ & $2.11(0.012)$ & $2.20(0.051)$ & $2.66(0.025)$ \\
\hline 0.80 & $24.25(0.020)$ & $23.79(0.019)$ & $10.89(0.494)$ & $5.72(0.132)$ & $5.77(0.122)$ \\
\hline 0.85 & $24.67(0.022)$ & $24.51(0.036)$ & $24.14(0.023)$ & $8.43(0.539)$ & $8.30(0.096)$ \\
\hline 0.90 & $25.00(0.041)$ & $25.12(0.031)$ & $25.28(0.054)$ & $26.43(0.165)$ & $27.05(0.248)$ \\
\hline 0.95 & $25.21(0.023)$ & $25.92(0.017)$ & $26.32(0.026)$ & $28.02(0.489)$ & $33.13(0.188)$ \\
\hline
\end{tabular}

Equivalently, $\Psi_{n}(\theta) \stackrel{P}{\rightarrow} \infty$, which means that the probability that any $\theta \neq \theta_{0}$ falls outside our proposed honest confidence interval converges to 1 . The proof of this lemma is given in Section 3 of Supplement A (Proposition 3.2) which also contains additional discussions and speculations on the $\Psi_{n}(\theta)$ based confidence sets.

Finally, to construct a confidence interval using the $\Psi$-statistic under long-range dependence, one will often need an estimate of $H$. For a known $H$, recall the conservative confidence interval given by $I_{n}=\left[\widetilde{C}_{L}(\alpha), \widetilde{C}_{U}(\alpha)\right]$ and denote the corresponding interval using $\hat{H}$, a consistent plug-in estimator of $H$, by $\hat{I}_{n}=\left[\hat{C}_{L}(\alpha), \hat{C}_{U}(\alpha)\right]$. If the quantiles of $\Psi$ are continuous as a function of $H$, again suggested strongly by extensive simulations, a simple application of Slutsky's lemma shows that $\liminf _{n \rightarrow \infty} P\left(\theta_{0} \in\left[\hat{C}_{L}(\alpha), \hat{C}_{U}(\alpha)\right]\right) \geq 1-\alpha$. Our simulation studies with estimated $H$, presented later, indicate that the above is indeed the case.

\subsection{Construction of Confidence Bands}

We now describe how our proposed methodology can be extended to construct conservative confidence bands for the monotone trend under short-range dependent errors. We also briefly discuss the long-range dependence case. Define $L_{n}(\theta, t)$ to be the $L_{n}$ test statistic for testing $H_{0}: m(t)=\theta$ and let $T_{n}(\theta, t), \Psi_{n}(\theta, t)$ be defined similarly. First, consider the problem of constructing simultaneous confidence intervals for the function $m$ at $k$ fixed points $0<a=t_{1} \leq t_{2} \leq \cdots \leq t_{k}=b<1$. By Theorem 3.1 from Supplement A, $\left\{L_{n}\left(\theta, t_{i}\right)\right\}_{\theta}$ for $i=1, \ldots, k$ are asymptotically independent as processes in $\theta$ under both short and long-range dependence provided the errors in the time-series model have Gaussian distributions and in the rest of this section we work under this (Gaussian) assumption.

Now, for each $1 \leq i \leq k$, use the method of inversion on $\left\{L_{n}\left(\theta, t_{i}\right)\right\}$ to construct an asymptotic $(1-\alpha)^{1 / k}$-level confidence interval $\left(l_{i, n}, u_{i, n}\right):=\left\{\theta: L_{n}\left(\theta, t_{i}\right) \leq F_{\mathbb{L}}^{\leftarrow}\left((1-\alpha)^{1 / k}\right)\right\}$ for $m\left(t_{i}\right)$. Since $P\left(\left(l_{i, n}, u_{i, n}\right) \ni m\left(t_{i}\right)\right) \rightarrow(1-\alpha)^{1 / k}$ and each $\left(l_{i, n}, u_{i, n}\right)$ is a function of $\left\{L_{n}\left(\theta, t_{i}\right)\right\}_{\theta}$, the pairs $\left\{l_{i, n}, u_{i, n}\right\}_{i=1}^{k}$ are asymptotically independent and

$$
\lim _{n \rightarrow \infty} P\left(\left(l_{i, n}, u_{i, n}\right) \ni m\left(t_{i}\right) \forall i\right)=\lim _{n \rightarrow \infty} \prod_{i=1}^{k} \mathbb{P}\left(\left(l_{i, n}, u_{i, n}\right) \ni m\left(t_{i}\right)\right)=(1-\alpha) .
$$


Next, we extend this approach to construct a confidence band for the function $m$. The first step is to monotonize the sequences $l_{i, n}$ and $u_{i, n}$, i.e., define $\tilde{l}_{1, n}=l_{1, n}$ and $\tilde{l}_{i, n}=\min \left(\tilde{l}_{i-1, n}, l_{i, n}\right)$ for $i \geq 2$ and similarly $\tilde{u}_{1, n}=u_{1, n}$ and $\tilde{u}_{i, n}=\max \left(\tilde{u}_{i-1, n}, u_{i, n}\right)$ for $i \geq 2$. On $[a, b] \subset(0,1)$, define the functions $l_{n}$ and $u_{n}$ as $l_{n}(t)=\tilde{l}_{i, n}$ if $t \in\left[t_{i}, t_{i+1}\right)$ and $u_{n}(t)=\tilde{u}_{i+1, n}$ if $t \in\left(t_{i}, t_{i+1}\right]$.

Proposition 5.3. The pair of functions $\left(l_{n}(t), u_{n}(t)\right)$ gives a conservative $100(1-\alpha) \%$ asymptotic confidence band for the function $m$ on the interval $[a, b]$, i.e., $\liminf _{n \rightarrow \infty} \mathbb{P}\left(\left(l_{n}(t), u(t)\right) \ni m_{n}(t) \forall t \in[a, b]\right) \geq(1-\alpha)$.

Remark 5.2. We construct confidence intervals on a sub-interval of the domain away from the boundaries, since isotonic estimates are known to be inconsistent at boundaries. See, for example Woodroofe and Sun (1993) [44]. Confidence bands may be constructed in a similar fashion using the statistics $\Psi_{n}(\theta, t)$ and $T_{n}(\theta, t)$. While the asymptotic independence is rigorously established under Gaussian errors (which simply entails showing that the asymptotic covariance goes to 0), we believe that it is true under more general errors; however, this appears to be a challenging problem.

Proof. By construction, for $i=1,2, \ldots, k$, we have:

$$
l_{n}\left(t_{i}\right)=\tilde{l}_{i, n} \leq l_{i, n} \leq m\left(t_{i}\right) \leq u_{i, n} \leq \tilde{u}_{i, n}=u_{n}\left(t_{i}\right) .
$$

For any $t \in\left(t_{i}, t_{(i+1)}\right)$ we have $m(t) \geq m\left(t_{i}\right) \geq l_{n}\left(t_{i}\right)=l_{n}(t)$ and $m(t) \leq m\left(t_{i+1}\right) \leq u_{n}\left(t_{i+1}\right)=u_{n}(t)$. Therefore

$$
\begin{aligned}
\mathbb{P}\left(\left(l_{n}(t), u_{n}(t)\right) \ni m(t), \forall t \in[a, b]\right) & =P\left(\left(l_{n}\left(t_{i}\right), u_{n}\left(t_{i}\right)\right) \ni m\left(t_{i}\right), \forall i=1, \ldots, k\right) \\
& \geq P\left(\left(l_{i, n}, u_{i, n}\right) \ni m\left(t_{i}\right), \forall i=1, \ldots, k\right),
\end{aligned}
$$

and the last probability converges to $(1-\alpha)$ by the discussion above.

We show in the simulation section that our method performs reasonably well for a number of different models under short-range dependent Gaussian errors. Note that as $L_{n}(\theta, t)=n \mathbb{L}_{n}(\theta, t) / \sigma_{n}^{2}$ and $\sigma_{n}^{2} \sim n \tau^{2}$ under short-range dependence, our implementation requires using the estimate $\hat{\tau}^{2}$ introduced previously in connection with the point-wise confidence intervals.

From the theoretical perspective, our method relies on a fixed number of points $k$ in the time-domain. However, for a meaningful practical implementation of this method, we would like to choose larger values of $k$ for larger data-sets. A rule of thumb is to take the number $k$ to be no larger than the order of the number of jump-points of the isotonic estimator (equivalently the number of flat stretches of the same) for a data-set of size $n$. This number is of the order $n^{1 / 3}$ under independence of errors or short-range dependence; indeed, this is what we use in our simulation experiments, keeping the points equi-spaced. Indeed, we believe that if the number of (equi-spaced) points $k$ is allowed to grow with $n$ at a rate slightly slower than $n^{1 / 3}$, the $L_{n}\left(\theta, t_{i}\right)^{\text {'s will }}$ continue to be asymptotically independent as processes and the conservative confidence band argument can be extended. However, a full-fledged justification of this is expected to require substantial additional developments of theoretical tools even in the i.i.d. case and is beyond the scope of the paper. 
We next briefly comment on the long-range dependence case. A similar strategy combining finitely many point-wise CIs can be pursued here as well under Gaussian errors. However, empirical simulations using the above strategy with $L_{n}$ and $\Psi_{n}$ both yield unsatisfactory confidence bands (not reported): with $L_{n}$, one must contend with the estimation of $m^{\prime}\left(t_{i}\right)$ which leads to coverage problems even for point-wise CIs, while, on the other hand, due to the lack of structure in the shape of the $\Psi$-statistic (for example, the nice path properties of $L_{n}$ and $T_{n}$ in $\theta$ as described in Lemma 3.2 of Supplement A do not hold for $\Psi_{n}$ ), the confidence bands constructed using $\Psi_{n}\left(\theta, t_{i}\right)$ are generally extremely conservative (and therefore un-informative). To the best of our understanding, the confidence bands question under LRD using isotonic methods remains a hard open problem.

\section{Simulation and Data Analysis}

\subsection{Performance of Confidence Intervals}

To study the performance of our confidence intervals we consider two choices for $m(t)$, namely:

$$
m_{1}(t)=e^{t} \quad \text { and } \quad m_{2}(t)= \begin{cases}t, & t \in(0,1 / 4] \\ 1 / 4+20000(t-1 / 4)^{2}, & t \in(1 / 4,1 / 4+1 / 200] \\ t+3 / 4, & t \in(1 / 4+1 / 200,1] .\end{cases}
$$

Observe the capricious behavior of $m_{2}$ in the interval $(1 / 4,1 / 4+1 / 200]$, where the function grows rapidly. We choose the midpoint $t_{0}=1 / 4+1 / 400$ from this interval. For $m_{1}$ we choose $t_{0}=1 / 2$.

In the following sections we demonstrate that our confidence intervals outperform existing methods for both conventional and challenging trend functions such as $m_{1}$ and $m_{2}$ respectively. We also show that the intervals perform well under both short and long-range dependent errors.

Data were generated from the models $y_{i}=m_{j}(i / n)+\epsilon_{i}$, for $i=1,2, \ldots, n$, and $j=1,2$. The errors were generated from different ARMA processes, fractional Gaussian noise for different Hurst indices and a FARIMA process. The marginal variance of the errors was 0.2 in all cases. Three statistics: $R_{n}$, $L_{n}$ (equivalently $\mathbb{L}_{n}$ ) and the IRE (defined in (2)) were used to construct confidence intervals for $m_{1}(0.5)$ in the first case, and $m_{2}(0.25+1 / 400)$ in the second. To use IRE, we constructed Wald-type confidence intervals based on the results of AH[2] (see Theorem 3) and ZW[47]. The required quantiles for this method can be found in Groeneboom (1989)[16] for the weak dependence case. For long-range dependent errors, we simulated (approximations to) the quantiles for some specific values of $H$. The average length and coverage of $90 \%$ confidence intervals based on 1000 repetitions were reported for various sample sizes $(n)$.

Constructing confidence intervals using $R_{n}$ is straightforward and follows the method outlined in the previous section. In order to use $L_{n}$ and the IRE, estimates of $\tau^{2}$ and $m^{\prime}\left(t_{0}\right)$ (only for the IRE) were needed under short-range 
dependence, while estimates of $m^{\prime}\left(t_{0}\right), \eta_{1}$ and $H$ were required for long-range dependence. Note that $\eta_{1}^{2}=\sigma^{2}$ is simply the common variance of the errors. Estimation of $\tau^{2}$ in the short-range dependence case has been already discussed. In the long-range dependence case, for FGN errors, $\sigma^{2}$ was estimated by the empirical variance of the $Y_{i}$ 's and in the case of FARIMA, using the approximate maximum likelihood method discussed in Haslett and Raftery (1989)[17]. Estimation of $m^{\prime}\left(t_{0}\right)$ is the most challenging part. Even for i.i.d. data, principled estimation in the monotone function setting is challenging - see Section 3.1 of Banerjee and Wellner (2005) for a discussion - and the difficulties are only exacerbated under dependence. Kernel based estimation, as in Banerjee and Wellner (2005) was used; thus,

$$
\hat{m}^{\prime}\left(t_{0}\right)=\frac{1}{h} \int K\left(\frac{t_{0}-t}{h}\right) d \hat{m}_{n}(t)
$$

where $h$ is the bandwidth and $K$, a Gaussian kernel. Two different bandwidth selection methods were considered: the method of cross-validation (see Section 5 from Supplement A), and, for short-range dependent data, over-smoothing with respect to the order of the spacing of the jumps $\left(n^{-1 / 3}\right)$ using the theoretically optimal bandwidth $n^{-1 / 7}$ for estimating the derivative of a monotone function by kernel smoothing its nonparametric MLE (see Groeneboom \& Jongbloed (2002) [14], Theorem 5.1 (iii)).

Estimation of $H$ : As seen above, estimation of $H$ is typically necessary for constructing confidence intervals using both $\mathbb{L}_{n}$ and $R_{n}$ under long-range dependence. In our simulations and data analysis we used the wavelet based Whittle estimator proposed in Moulines et. al. (2008)[24] for $H$. (For other methods see Abry and Veitch (1998)[1], Stoev et. al. (2005)[39], Fay et. al. (2009)[12] and Doukhan et. al. (2003)[10].) There are two advantages of using this approach. First, the wavelet based method is invariant to smooth polynomial trends in the data up to a given order $k$, where $k$ is the number of zero moments of the mother wavelet function. This is because the discrete wavelet transform amounts to convolving the data with a low-pass filter, which acts similarly to finite differencing operators of order $k$. Thus, the wavelet coefficients and hence the Hurst parameter estimator are unaffected by adding to the data any polynomial trend of degree up to $k$. Although irregular (or higher order) trends do affect the wavelet estimators, in practice, the aforementioned zero-moment property minimizes the effect of the unknown monotone function $m$ on the estimation of $H$. For more details and the effect of irregularities in the trend on the wavelet estimators of $H$, see Stoev et. al. (2005)[39]. (We used wavelets of order $k=7$ for this paper.) Secondly, the estimate of $H$ obtained by this method is known to be consistent at a polynomial rate under rather general conditions (see Corollary 4 of Moulines et. al., 2008 [24]). This result allows us to use the $\mathbb{L}_{n}$ statistic under long-range dependence (see Section 5.2, above).

Also, note that the dependence of $\Psi_{n}$-based inference on $H$ is minimal in the sense that $H$ is only required to determine the cut-off value for inversion and does not enter into the computation of $\Psi_{n}$ itself (unlike what happens with 
the IRE or $L_{n}$ ). Hence, if there were a general nesting of quantiles of $\Psi$ with respect to $H$, one could have built conservative confidence intervals at any given level without estimating $H$ ! Such type of robustness to long-range dependence is too much to hope for. Nevertheless, while the nesting property is absent in general, at both $90 \%$ and $95 \%$ levels, our estimated quantiles increase as a function of $H$ for $0.5 \leq H \leq 0.95$, as a quick inspection of Table I (and more extensive simulations not reported here) reveals. This empirical observation can, therefore, be used to construct conservative $\Psi_{n}$-based confidence intervals at these two levels, by using the quantiles corresponding to $H=0.95$. Values of $H$ greater than 0.95 indicate extreme levels of long-range dependence, which should be dealt with care, but are rarely encountered in practice. Note that such conservative CI's are completely agnostic as to whether the underlying dependence is short- or long-range, exemplifying the robustness of our method. The bottomline here is that if little is qualitatively known about the extent of dependence, it is better to go with the conservative intervals above, whereas if reasonably reliable information about the error structure is available, the best distributional approximation to the $\Psi$-statistic (generally at the expense of estimating $H$ ) should be used.

Discussion of the simulation results:

- Short-range dependence regime: From Tables 1 and 2 in Supplement A, we observe that for short-range dependent errors, $L_{n}$ and $\Psi_{n}$ are performing much better, in terms of coverage, than the Wald-type confidence intervals based on the IRE. Note that the IRE based CIs show systematic under-coverage, especially for $m_{2}$, as the derivative estimation procedure is highly unstable in this situation. The $L_{n}$ and $\Psi_{n}$ based intervals both exhibit coverage much closer to the nominal, though the $\Psi_{n}$ based ones tend to over-cover, which can be attributed to the manner of their construction; see the comments following Proposition 5.1. The average lengths of the CIs using $\Psi_{n}$ are also somewhat larger than their $L_{n}$ based counterparts. Since accurate estimators of $\tau^{2}$ are often available, in applications, we recommend using $L_{n}$ whenever possible, i.e. unless we have very little information about the dependence structure of the errors, or if the dependence structure involves estimating too many parameters compared to sample size.

- Long-range dependence regime: Tables 3,4 and 5 in Supplement A report simulation results (for CIs) based on $\Psi_{n}, L_{n}$ and IRE for long-range dependent errors using the true value of $H$ but estimating all other parameters wherever necessary. We see that the $\Psi_{n}$ based method outperforms both $L_{n}$ and the IRE based methods, in terms of coverage, as is evident from Tables3, 4 and 5 in Supplement A, with the latter intervals showing systematic under-coverage, especially at higher values of $H$ and under FARIMA errors. While $L_{n}$ was seen to be reliable in the short-range dependence case, its performance suffers under long-range dependence because the derivative $m_{2}^{\prime}\left(t_{0}\right)$ now needs to be estimated for the corresponding CIs. For function $m_{2}$, the coverage of the IRE based CIs worsens significantly, owing to reasons similar to the short-range dependence case. The average lengths of the intervals using $\Psi_{n}$ are consistently larger than those from the other methods, showing that the lengths of asymptotically pivotal $\Psi_{n}$-based CIs adapt nicely to the underlying variability in order to maintain close-to-nominal coverage. Additional 
Table 2: Coverage of $90 \%$ Confidence Bands

\begin{tabular}{|l|l|c|c|}
\hline Function & \multicolumn{1}{|c|}{ Errors } & $\mathrm{n}$ & Coverage \\
\hline$m(t)=1 /\left(1+e^{-20(t-0.5)}\right)$ & $\operatorname{AR}(2)$ coeff $(0.7,-0.6)$ & 2000 & $92.5 \%$ \\
\hline$m(t)=1 /\left(1+e^{-20(t-0.5)}\right)$ & $\operatorname{AR}(2)$ coeff $(0.7,-0.6)$ & 5000 & $91.9 \%$ \\
\hline$m(t)=1 /\left(1+e^{-20(t-0.5)}\right)$ & ARMA $(1,1)$ coeff $(0.8,0.4)$ & 2000 & $91.0 \%$ \\
\hline$m(t)=1 /\left(1+e^{-20(t-0.5)}\right)$ & ARMA $(1,1)$ coeff $(0.8,0.4)$ & 5000 & $90.4 \%$ \\
\hline$m(t)=t^{2}$ & AR $(2)$ coeff $(0.7,-0.6)$ & 2000 & $93.5 \%$ \\
\hline$m(t)=t^{2}$ & AR(2) coeff $(0.7,-0.6)$ & 5000 & $91.7 \%$ \\
\hline$m(t)=t^{2}$ & ARMA $(1,1)$ coeff $(0.8,0.4)$ & 2000 & $92.3 \%$ \\
\hline$m(t)=t^{2}$ & ARMA $(1,1)$ coeff $(0.8,0.4)$ & 5000 & $92.5 \%$ \\
\hline
\end{tabular}

simulations (not reported here) were run to assess the performance of oracle $L_{n}$-based CIs, constructed using the true values of the nuisance parameters. It was seen that such oracle $C I$ s are substantially better: close-to-nominal coverage was restored and the average lengths were now less than the $\Psi_{n}$-based CIs. Of course, the oracle CIs are not available in practice, but the experiments underscore the importance of (asymptotic) pivotality.

Since the performance of $L_{n}$ and IRE were not terribly satisfactory assuming $H$ known, we refrain from presenting results for estimated $H$ (which only worsens the performance). Table 6 in Supplement A demonstrates the performance of the $\Psi$-statistic based CIs for $m_{1}$ using the estimated $H$ to determine the critical value. The performance of the $C I$ s is seen to be very reasonable.

Finally, in view of our discussion, we recommend using $L_{n}$ or $T_{n}$ under short-range dependence unless the covariance is difficult to estimate. For short-range dependent data where the covariance is difficult to estimate or if one suspects the presence of long-range dependence, we recommend using $\Psi_{n}$ to construct confidence intervals.

\subsection{Performance of Confidence Bands Under SRD}

To study the performance of the confidence bands proposed in Section 5.3 we used two choices for the trend function namely $m_{1}(t)=1 /\left(1+e^{-20(t-0.5)}\right)$ and $m_{2}(t)=t^{2}$. We used two different dependence structures for errors. The first one is an $\operatorname{AR}(2)$ model with $\mathrm{AR}$ coefficients 0.7 and -0.6 , for the second dependence structure we used an ARMA $(1,1)$ model with AR coefficient 0.8 and MA coefficient 0.4. The marginal variance for both the structures was taken to be 0.2 . Table 2 presents simulated coverage of (conservative) $90 \%$ confidence bands calculated from 1000 iterations for sample sizes $n=2000$ and $n=5000$. We chose 13 and 17 equidistant points (starting from the 10-th data-point) respectively for sample sizes 2000 and 5000 (i.e. roughly $n^{1 / 3}$ ) to construct the confidence band. As seen from Table 2, the bands constructed by this method give reasonable coverage.

Figure 2 shows confidence bands for simulated datasets from the isotonic 
regression model with trend functions $m_{1}$ and $m_{2}$ and errors coming from the $\mathrm{AR}(2)$ model above. Both data-sets have $n=2000$ data-points and we used 13 equally spaced points to construct the confidence bands.

Note that our confidence bands are not smooth but staircase-shaped. Indeed, given our minimal assumptions on the underlying monotone function - namely continuous differentiability - one cannot hope for smoother confidence bands than the staircase-shaped ones. A smoother confidence band would require reliable bounds on the derivative of the function on the interval over which the band is sought to be constructed: such bounds would restrict the rate at which the function could increase over the domain and this information could then be used to modify/smoothen the stair-case shaped bands.

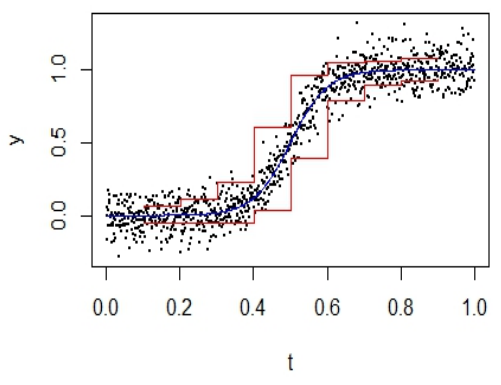

(a) $m_{1}(t)=1 /\left(1+e^{-20(t-0.5)}\right)$

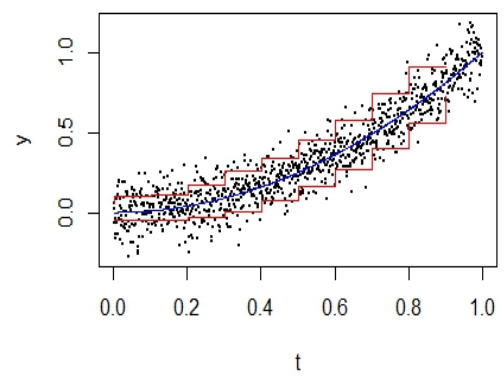

(b) $m_{2}(t)=t^{2}$

Figure 2: $\quad 90 \%$ confidence bands

\subsection{Analysis of Global Temperature Anomaly and Internet Usage Data}

We apply our methodology to two data sets, one exhibiting short- and the other long-range dependence. In the former case we use the $L_{n}$ statistic and in the latter the ratio based statistic $\Psi_{n}$.

Global Temperature Anomaly Data (Short-range dependence) We consider the global warming data used in ZW[47], which consists of global annual temperature anomalies, measured in degrees celsius from 1850 to 2009. These anomalies are, simply, temperature deviations measured with respect to the base period 1961-1990. The autocorrelation plot of these data suggests that the dependence can be well accounted for using an $\operatorname{AR}(2)$ process, see ZW[47]. The short-range dependence condition (6) applies to AR(2) time series. Figures 3(a) and 3(b) represent the data along with its isotonic regression estimates and $90 \%$ confidence intervals and band respectively obtained by using $L_{n}$. The estimate of the asymptotic standard deviation $\tau$ 


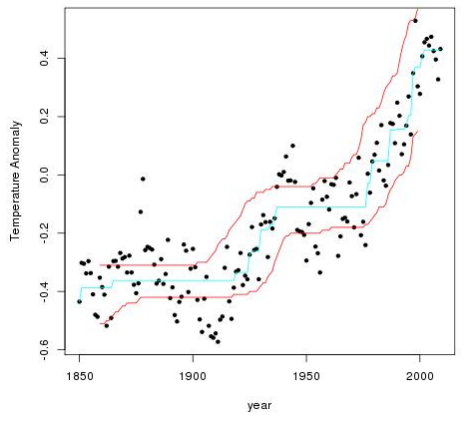

(a) Point-wise confidence intervals

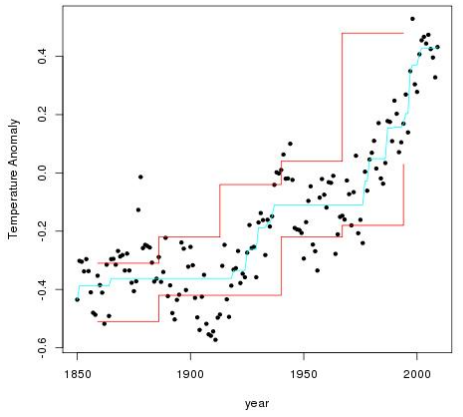

(b) Confidence band

Figure 3: 90\% confidence intervals and confidence band for global temperature anomaly data

was taken to be 0.1248 from $\mathrm{Wu}$ et. al. (2001)[45]. Note that the point-wise confidence intervals in the left panel form a rather smooth band, which mimics, in shape, the isotonic regression curve but should not be confused with the uniform confidence band in the right panel which is naturally wider. Apart from the estimate of $\tau$, our methodology is completely agnostic to the nature of short-range dependence and regularity of the trend. As can be seen from the two figures, our confidence intervals show some (data-based) evidence for systematic growth of the global temperature anomalies, as should be expected, given the compelling evidence from numerical climate model simulations of the Intergovernmental Panel of Climate Change IPCC (2007)[19]. We compare our point-wise confidence intervals method with those obtained by kernel smoothing ignoring the monotonicity constraint as decribed in Robinson (1997)[33]. Following the example described in Section 5 of that paper, we use the kernel $k(x)=[1 / 2(1+\cos (\pi x))] \mathbf{1}(|x| \leq 1)$ and the three fixed bandwidths used therein: $b=.05, .075, .1$ for the regression estimator. We use the differencing method, equation (4.6) (with the same kernel $l=k$ and the same three bandwidths $(c=b)$ ), along with the formulas in equation (4.10) of that paper to estimate the asymptotic variance. Note that

Robinson's set-up assumes Lipschitz continuity of the regression function and its derivative, which is stronger than the assumptions underlying our isotonic procedure. Figure 4(b) shows these point-wise CIs for $b=c=0.05$ (the other bandwidths produced similar results) with the isotonic point-wise CIs being plotted alongside in Figure 4(a) for convenience of comparison. The pattern of the CIs using Robinson's method is similar to those obtained by the isotonic procedure for the latter half of the time period when the monotonicity of the trend is more pronounced. The different behavior of the two estimators in the earlier time period can be accounted for by noticing that our procedure enforces global monotonicity while Robinson's does not. 


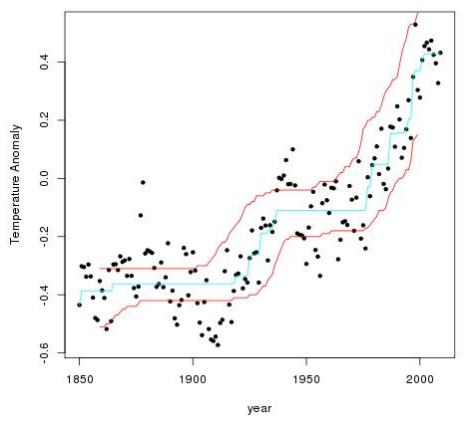

(a) Point-wise confidence intervals us- (b) Point-wise confidence intervals using ing $L_{n}$

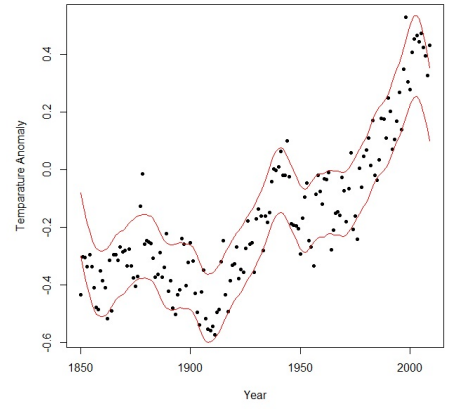

kernel smoothing

Figure 4: 90\% point-wise confidence intervals for global temperature anomaly data

Internet Traffic Data (Long-range dependence) This example involves computer network traffic data obtained from the Internet2 network [3]. The data consists of number of bytes per 100 millisecond time-intervals over a fast backbone link measured on 17th March, 2009. Such traffic traces exhibit typical diurnal patterns with clearly defined periods of monotone non-decreasing or non-increasing trends throughout the day. This is associated with usual growth/decay of the number of active users in the beginning/middle of the day. Further, it is well known and documented that Internet traffic traces exhibit long-range dependence (see e.g. Willinger et. al. (1995)[43] and Stoev et. al. (2005)[39]). Such data provide an ideal test-bed for the performance of our confidence intervals based on the ratio statistic. To be able to provision network capacity as well as detect anomalous network activity, it is important to have accurate estimates of confidence intervals that are robust to the presence of long-range dependence and account for natural traffic trends, without imposing stringent parametric/smoothness assumptions. This is particularly important in the network traffic context, where unusual changes in the regularity of the trend may occur and methods that involve estimation of derivatives require great care to implement and, in fact, can lead to non-robust interval estimates.

We focused on the time period $11: 06$ to $16: 36$ GMT, corresponding to $7: 06$ AM to $12: 36$ AM in US EST, where there is a typical monotone non-decreasing diurnal trend due to systematic increase of the number of active users in the beginning of the day. The Hurst parameter was estimated to be $\hat{H}=0.9491$ using wavelet methods[39].

Figure 5(a) shows $90 \%$ point-wise confidence intervals at 50 time points based on the $\Psi_{n}$ statistic with $H=0.95$. Observe that the confidence intervals (which are joined across time) generally track the monotone trend but should not be interpreted as uniform bands. In contrast to the intervals based on the 


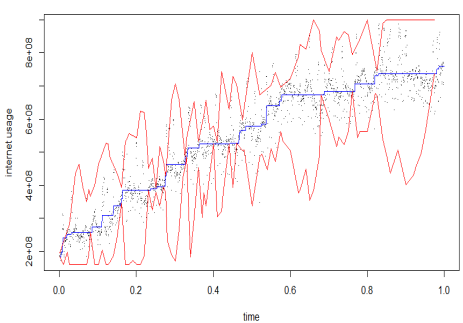

(a) Point-wise confidence intervals using $R_{n}$

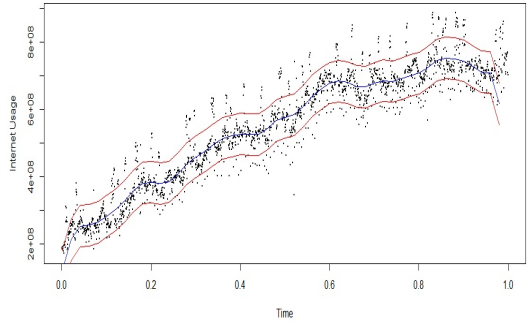

(b) Point-wise confidence intervals using kernel smoothing

Figure 5: 90\% point-wise confidence intervals for internet traffic data

$L_{n}$ and $T_{n}$ statistics, the intervals based on $\Psi_{n}$ are not smooth over time. Since we have not performed any smoothing, nor aimed to produce a confidence band, this feature is not alarming. This irregularity of the proposed confidence intervals is yet to be fully understood, theoretically. Since $\Psi_{n}$ is obtained as a non-linear, ratio functional of the discrepancy statistics $L_{n}$ nor $T_{n}$, the joint behavior of $L_{n}$ and $T_{n}$ near zero should play a key role.

Regardless of the unusual irregularity feature, our confidence estimates provide reliable coverage and are nearly dependence-universal, as confirmed by our theory and extensive simulation studies only some of which are reported. We compare our intervals to Figure 5(b) which provides point-wise confidence intervals at the same 50 time points using the method in Robinson (1997) [33]. The details of the construction remain the same as in the short-range dependence case described previously, the only difference being that $\hat{H}$ and $\hat{G}$ appearing in the asymptotic variance are now computed using the raw data, i.e. $\hat{u}_{t}=Y_{t}$ as in (4.12) of Robinson's paper instead of the formula (4.6) used under short-range dependence. The estimated $\hat{H}$ based on the alternative method is 0.9502 , close to our obtained estimate 0.9491 . The most striking difference between this kernel method and ours is that the length of the confidence intervals constructed by the kernel method remains the same for all the 50 points, since the asymptotic variance of the estimator of $m(x)$ does not depend on $x$ therein. Our confidence intervals appear to be reacting more keenly to the variability in the data relative to the slope of the trend curve. Section 6 and Table 7 in Supplement A present some limited studies of the coverage properties of the kernel based CIs for the same two functions $m_{1}$ and $m_{2}$ in (21), which we used to study our proposed methods. As is evident from the table, the kernel based CIs suffer from considerable under-coverage for the ill-behaved $m_{2}$ and especially so under strong dependence, similar to the IRE based intervals reported in other tables. Thus, while being visually more appealing (smother) than the $\Psi_{n}$ based CIs, the kernel based CIs are not accurate for ill-behaved trend functions and the $\Psi_{n}$ based ones remain substantially more reliable in such cases. 


\section{A Proof-sketch of asymptotics of the test statistics $L_{n}$ and $T_{n}$ :}

The statistics $L_{n}$ and $T_{n}$ are determined by the discrepancy between $\hat{m}_{n}(t)$ and $\hat{m}_{n}^{0}(t)$ in the neighborhood of $t_{0}$ over which they differ.

We focus on a shrinking neighborhood of $t_{0}$ at rate $d_{n} \downarrow 0$, which will determined by the type of dependence structure of the error sequence, since $\hat{m}_{n}$ and $\hat{m}_{n}^{0}$ are equal outside of neighborhoods of this order of magnitude. For example, under independence or short-range dependence $d_{n} \sim n^{-1 / 3}$, while under long-range dependence the rate will involve the Hurst index. More formally, let $z:=d_{n}^{-1}\left(t-t_{0}\right)$ and define

$$
X_{n}(z)=\frac{1}{d_{n}}\left(\hat{m}_{n}\left(t_{0}+z d_{n}\right)-\theta_{0}\right) \text { and } Y_{n}(z)=\frac{1}{d_{n}}\left(\hat{m}_{n}^{0}\left(t_{0}+z d_{n}\right)-\theta_{0}\right),
$$

for $z \in\left(a_{n}, b_{n}\right]:=\left(-d_{n}^{-1} t_{0}, d_{n}^{-1}\left(1-t_{0}\right)\right]$. Here $\theta_{0}=m\left(t_{0}\right)$. It turns out that the statistics $L_{n}$ and $T_{n}$ can be represented asymptotically as fairly simple integrals involving $X_{n}$ and $Y_{n}$ and also that the set of $z$ 's on which they differ is contained, with high probability, in a compact set. These are the contents of the two results stated below.

Proposition A.1. For $L_{n}$ and $T_{n}$ as in (4), we have

$$
\begin{aligned}
L_{n} & =\frac{n^{2} d_{n}^{3}}{\sigma_{n}^{2}}\left(\int_{\left(a_{n}, b_{n}\right]}\left(X_{n}^{2}(z)-Y_{n}^{2}(z)\right) d z+o_{P}(1)\right) \\
T_{n} & =\frac{n^{2} d_{n}^{3}}{\sigma_{n}^{2}}\left(\int_{\left(a_{n}, b_{n}\right]}\left(X_{n}(z)-Y_{n}(z)\right)^{2} d z+o_{P}(1)\right) .
\end{aligned}
$$

Lemma A.1. Let $D_{n}:=\left\{z \in \mathbb{R}: X_{n}(z) \neq Y_{n}(z)\right\}$. For any $\epsilon>0$, there exist $M_{\epsilon}>0$ and $n_{\epsilon}>0$, such that

$$
\mathbb{P}\left(D_{n} \subset\left[-M_{\epsilon}, M_{\epsilon}\right]\right) \geq 1-\epsilon,
$$

for all $n \geq n_{\epsilon}$.

The proofs of the preceding proposition and lemma are given in Section 2.2 of Supplement A.

It is then clear that fathoming the asymptotic behavior of $L_{n}, T_{n}$ requires an understanding of the asymptotic behavior of the processes $\left(X_{n}, Y_{n}\right)$ on compact sets, since with high probability, the difference set $D_{n}$ is contained in a compact set. If we could show that $\left(X_{n}, Y_{n}\right)$ converge to limit processes $\left(X_{\infty}, Y_{\infty}\right)$ with increasing sample size on every compact set (in a strong-enough metric under which integral type functionals are continuous), then, roughly speaking, up to adequate normalizations our limits for $L_{n}$ and $T_{n}$ should have forms:

$$
\int\left(X_{\infty}^{2}(z)-Y_{\infty}^{2}(z)\right) d z \text { and } \int\left(X_{\infty}(z)-Y_{\infty}(z)\right)^{2} d z
$$


respectively. It turns out that the topology of $L^{2}$ convergence on compact sets is adequate for this purpose.

The processes $X_{n}$ and $Y_{n}$ can be represented as greatest convex minorant functionals of a normalized version of the the process $U_{n}$, the linear interpolation of the cumulative sum process of the $Y_{i}$ 's, namely:

$$
U_{n}(t)=\frac{Y_{1}+Y_{2}+\cdots+Y_{\lfloor n t\rfloor}}{n}+\frac{(n t-\lfloor n t\rfloor)}{n} Y_{\lfloor n t\rfloor+1}, t \in[0,1] .
$$

More specifically, defining:

$$
\mathbb{V}_{n}(z):=d_{n}^{-2}\left(U_{n}\left(t_{0}+d_{n} z\right)-U_{n}\left(t_{0}\right)-m\left(t_{0}\right) d_{n} z\right), \quad z \in\left(a_{n}, b_{n}\right],
$$

we can write:

$$
\begin{aligned}
X_{n}(z)= & \mathcal{L} \circ \mathcal{T}_{\left(a_{n}, b_{n}\right]}\left(\mathbb{V}_{n}\right)(z) \\
Y_{n}(z)= & \left(\mathcal{L} \circ \mathcal{T}_{\left(a_{n}, l_{n}\right]}\left(\mathbb{V}_{n}\right)(z) \wedge 0\right) \mathbf{1}_{\left(a_{n}, l_{n}\right]}(z)+0 \times \mathbf{1}_{\left(l_{n}, 0\right]}(z) \\
& +\left(\mathcal{L} \circ \mathcal{T}_{\left(l_{n}, b_{n}\right]}\left(\mathbb{V}_{n}\right)(z) \vee 0\right) \mathbf{1}_{\left(0, b_{n}\right]}(z),
\end{aligned}
$$

where $l_{n}=d_{n}^{-1}\left(t_{l}-t_{0}\right)$. (Recall that the operators involved in the definition of $X_{n}$ and $Y_{n}$ were introduced in Section 4.) This is a direct consequence of the well-known representation of $\hat{m}_{n}$ and $\hat{m}_{n}^{0}$ in terms of $U_{n}$ (see (2.1) in Supplement A) followed by an appropriate renormalization. The limiting properties of $\left(X_{n}, Y_{n}\right)$ are therefore driven by those of $\mathbb{V}_{n}$, which is fortunately well-studied (AH [2]). More concretely, we know:

Theorem A.1. Consider the processes $\mathbb{V}_{n}$ in the space $C(\mathbb{R})$ equipped with the topology of uniform convergence on compact sets. Then, as $n \rightarrow \infty$,

$$
\mathbb{V}_{n} \Longrightarrow\{\mathbb{G}(z)\}_{z \in \mathbb{R}} \equiv\left\{\mathbb{G}_{a, b}(z)\right\}_{z \in \mathbb{R}}:=\left\{a \mathbb{W}(z)+b z^{2}\right\}_{z \in \mathbb{R}},
$$

where $b=\frac{1}{2} m^{\prime}\left(t_{0}\right)$ and (i) (under weak dependence) $d_{n}=n^{-\frac{1}{3}}, \mathbb{W}$ is a two-sided Brownian motion on $\mathbb{R}$, and $a:=\tau$ given in (8).

(ii) (under strong dependence) $d_{n}=l_{2}(n) n^{-\frac{d}{2+d}}, \mathbb{W}$ is the fBm process $B_{H}$ and $a:=\left|\eta_{1}\right|$. (Here $l_{2}$ is a slowly varying function related to $l_{1}$ as shown in the proof of the theorem, provided in Supplement A.)

We therefore expect $\left(X_{\infty}, Y_{\infty}\right)$ the limits of $\left(X_{n}, Y_{n}\right)$ to be given by replacing $a_{n}, b_{n}, l_{n}, \mathbb{V}_{n}$ by their corresponding limits in (27). But these are precisely $\left(\mathcal{S}_{a, b}(z), \mathcal{S}_{a, b}^{0}(z)\right)$ introduced in Section 4.

We next define the space $L_{l o c}^{2}$ which appears in the formal statement of convergence of $\left(X_{n}, Y_{n}\right)$. This is the space of all functions which are square integrable on compact sets. The convergence in this space is accordingly defined, that is, a sequence of functions $f_{n} \rightarrow f$ as $n \rightarrow \infty$ in $L_{l o c}^{2}$ if $\int_{I}\left(f_{n}-f\right)^{2} \rightarrow 0$ as $n \rightarrow \infty$ for every compact interval $I$. In fact with this convergence the space is metrizable. We have:

Theorem A.2. As $n \rightarrow \infty$, we have

$$
\left\{\left(X_{n}(z), Y_{n}(z)\right)\right\}_{z \in \mathbb{R}} \Longrightarrow\left\{\left(\mathcal{S}_{a, b}(z), \mathcal{S}_{a, b}^{0}(z)\right)\right\}_{z \in \mathbb{R}} \quad \text { in } L_{l o c}^{2} \times L_{l o c}^{2}
$$


where the components of the limit process are defined in (12).

The formal proof of this theorem is highly technical and provided in

Supplement A. The above result coupled with the forms of the expressions in (24) immediately lead to the forms for $L_{a, b}$ and $T_{a, b}$ given in (13).

\section{B Proof of Theorem 4.2}

We first state a version of the converging together lemma which is used later, and is an adaptation of Theorem 8.6.2 in Resnick (1999)[32].

Lemma B.1. Let $\xi, \xi_{\delta, c, n}, \xi_{\delta, c}, \eta_{n}, n \in \mathbb{N}, \delta, c>0$ be random elements taking values in a metric space $(E, d)$. If (i) $\xi_{\delta, c, n} \Rightarrow \xi_{\delta, c}$, as $n \rightarrow \infty$, (ii) $\xi_{\delta, c} \Rightarrow \xi$, as $c \rightarrow \infty$ and $\delta \uparrow 0$ and (iii) for all $\epsilon>0$,

$$
\lim _{\delta \uparrow 0} \lim _{c \rightarrow \infty} \limsup _{n \geq 1} \mathbb{P}\left(d\left(\xi_{\delta, c, n}, \eta_{n}\right)>\epsilon\right)=0,
$$

then $\eta_{n} \Rightarrow \xi$, as $n \rightarrow \infty$.

Proof of Theorem 4.2 By Lemma A.1 and Theorem 3.1 (Supplement B), for every $\epsilon>0$ there exists an interval $K_{\epsilon}:=\left[-M_{\epsilon}, M_{\epsilon}\right]$ such that, for all large $n$,

$$
\mathbb{P}\left[D_{n} \subset\left[-M_{\epsilon}, M_{\epsilon}\right]\right]>1-\epsilon \text { and } \mathbb{P}\left[D_{a, b} \subset\left[-M_{\epsilon}, M_{\epsilon}\right]\right]>1-\epsilon .
$$

Let now

$$
\begin{gathered}
\xi_{\epsilon, n}=\left(\int_{K_{\epsilon}}\left(X_{n}^{2}(z)-Y_{n}^{2}(z)\right) d z, \int_{K_{\epsilon}}\left(X_{n}(z)-Y_{n}(z)\right)^{2} d z\right) \\
\xi_{\epsilon}=\left(\int_{K_{\epsilon}}\left(\left(\mathcal{S}_{a, b}(z)\right)^{2}-\left(\mathcal{S}_{a, b}^{0}(z)\right)^{2}\right) d z, \int_{K_{\epsilon}}\left(\mathcal{S}_{a, b}(z)-\mathcal{S}_{a, b}^{0}(z)\right)^{2} d z\right) .
\end{gathered}
$$

Also, let

$$
\begin{gathered}
\eta_{n}=\left(\int_{\left(a_{n}, b_{n}\right]}\left(X_{n}^{2}(z)-Y_{n}^{2}(z)\right) d z, \int_{\mathbb{R}}\left(X_{n}(z)-Y_{n}(z)\right)^{2} d z\right), \\
\xi=\left(\int_{D_{a, b}}\left(\left(\mathcal{S}_{a, b}(z)\right)^{2}-\left(\mathcal{S}_{a, b}^{0}(z)\right)^{2}\right) d z, \int_{D_{a, b}}\left(\mathcal{S}_{a, b}(z)-\mathcal{S}_{a, b}^{0}(z)\right)^{2} d z\right) .
\end{gathered}
$$

Since $K_{\epsilon}$ contains $D_{n}:=\left\{z: X_{n}(z) \neq Y_{n}(z)\right\}$ with probability greater than $1-\epsilon$ and $\left(a_{n}, b_{n}\right]$ grows up to $\mathbb{R}$, for large $n$, we have

$\lim _{\epsilon \downarrow 0} \lim \sup _{n>1} \mathbb{P}\left(\xi_{\epsilon, n} \neq \eta_{n}\right)=0$. We similarly have that $\lim _{\epsilon \downarrow 0} \mathbb{P}\left(\xi_{\epsilon} \neq \xi\right)=0$.

Finally, by Theorem A.2 and the continuous mapping Theorem, for all fixed $\epsilon>0$, we have $\xi_{\epsilon, n} \Rightarrow \xi_{\epsilon}$, as $n \rightarrow \infty$. Thus, all conditions of the converging together lemma (cf Lemma B.1) hold, where in this simple case there is no dependence on $\delta>0$. Hence $\eta_{n} \Rightarrow \xi, n \rightarrow \infty$, which, in view of Proposition A.1, yields

$$
\frac{\sigma_{n}^{2}}{n^{2} d_{n}^{3}}\left(L_{n}, T_{n}\right) \Longrightarrow\left(\mathbb{L}_{a, b}^{(H)}, \mathbb{T}_{a, b}^{(H)}\right)
$$


as $n \rightarrow \infty$.

To complete the proof, it remains to show that

$$
\left(\mathbb{L}_{a, b}^{(H)}, \mathbb{T}_{a, b}^{(H)}\right) \stackrel{d}{=} a^{2}\left(\frac{a}{b}\right)^{\frac{2 H-1}{2-H}}\left(\mathbb{L}^{(H)}, \mathbb{T}^{(H)}\right) .
$$

This follows from a scaling argument. Indeed, by the $H$-self-similarity of $B_{H}$, for $\mathbb{G}(z) \equiv \mathbb{G}_{a, b}^{H}(z)=a B_{H}(z)+b z^{2}$, we have

$$
\left\{\mathbb{G}_{a, b}^{H}(z)\right\}_{z \in \mathbb{R}} \stackrel{d}{=} a(a / b)^{\frac{H}{2-H}}\left\{\left(\mathbb{G}_{1,1}^{H}\left((b / a)^{\frac{1}{2-H}} z\right)\right)\right\}_{z \in \mathbb{R}}
$$

Thus, the process $\left\{\left(\mathcal{S}_{a, b}(z), \mathcal{S}_{a, b}^{0}(z)\right)\right\}_{z \in \mathbb{R}}$ equals in distribution

$$
a(b / a)^{\frac{1-H}{2-H}}\left\{\left(\mathcal{S}_{1,1}\left((b / a)^{\frac{1}{2-H}} z\right), \mathcal{S}_{1,1}^{0}\left((b / a)^{\frac{1}{2-H}} z\right)\right)\right\}_{z \in \mathbb{R}}
$$

which by substituting in (13) and making a change of variables yields (31).

Remark B.1. The result of Theorem 4.1 can be formally recovered from the statement of Theorem 4.2 by letting $H=1 / 2, a=\tau, d_{n}=n^{-1 / 3}$, using the fact that $\sigma_{n}^{2} / n \rightarrow \tau^{2}$ and noting that $\mathbb{L}^{(1 / 2)}$ and $\mathbb{T}^{(1 / 2)}$ are precisely the $\mathbb{L}$ and $\mathbb{T}$ of Theorem 4.1 respectively.

\section{Supplementary Materials}

Supplement A: Supplement containing technical details and proofs of the results stated in the paper

Supplement B: Technical report containing properties of some important functionals Brownian and fractional Brownian motion in context of shape restricted regression. This file also contains properties and simulation of the random variables appearing in the limit distribution of the statistics used in this paper.

\section{References}

[1] Abry, P. and Veitch, D. (1998) Wavelet analysis of long-range dependent traffic. Institute of Electrical and Electronics Engineers. Transactions on Information Theory Vol. 44, No. 1, 2-15.

[2] Anevski, D. and Hossjer, O.(2006) A general asymptotic scheme for inference under order restrictions. The Annals of Statistics Vol. 34, 1874-1930.

[3] Backbone link traces from the Internet2 network http://www.internet2.edu/

[4] Banerjee, M. and Wellner, J. A.(2001) Likelihood ratio tests for monotone functions. The Annals of Statistics Vol. 29, 1699-1731.

[5] Banerjee, M. and Wellner, J. A.(2005) Confidence intervals for current status data. Scandinavian Journal of Statistics Vol. 32 405-424. 
[6] Banerjee, M.(2007) Likelihood based inference for monotone response models. Annals of Statistics Vol 35, No. 3 931-956.

[7] Banerjee, M. (2009) Inference in exponential family regression models under certain shape constraints. Advances in Multivariate Statistical Methods, Statistical Science and Interdisciplinary Research, Vol. 4, 249-272. World Scientific.

[8] Brunk, H. D. (1970) Estimation of isotonic regression. In Nonparametric Techniques in Statistical Inference (M. L. Puri, ed,) 177-197. Cambridge University Press, London.

[9] Clifford, H., Lang, G. and Soulier, P . (2005). Estimation of long memory in the presence of a smooth nonparametric trend. J. Amer. Statist. Assoc., Vol 100, no. $471,853-871$.

[10] Doukhan, P., Oppenheim, G. and Taqqu, M. S. (2003). Theory and applications of long-range dependence., Birkhäuser Boston Inc., Boston, MA

[11] Fan, J. and Yao, Q. (2003) Nonlinear Time Series. Nonparametric and Parametric Methods Series: Springer Series in Statistics.

[12] Fay, G., Moulines, E., Roueff, F. and Taqqu, M. S. (2009). Estimators of long-memory: Fourier versus wavelets. Journal of Econometrics Vol. 151, No. 2 159-177.

[13] Groeneboom, P. (1985). Estimating a monotone density. Proceeding of the Berkeley Conference in Honor of Jezry Neyman and Jack Kiefer Vol II, (Lucien, M. LeCam and Richard A. Olshen eds.)

[14] Groeneboom, P. and Jongbloed, G. (2003). Density estimation in the uniform deconvolution model. Statistica Neerlandica Vol. 57 136-157.

[15] Groeneboom, P. and Wellner J.A. (1992). Information Bounds and Nonparametric Likelihood Estimation. Birkhauser, Basel.

[16] Groeneboom, P. and Wellner J.A. (2001). Computing Chernoff's distribution. Journal of Computational and Graphical Statistics. Vol. 10, 388-400.

[17] Haslett J. and Raftery A.E. (1989). Space-Time Modelling with Long-Memory Dependence: Assessing Ireland's Wind Power Resource. Applied Statistics Vol. 38, $1-50$.

[18] Hussian, M., Grimvall, A., Burdakov, O. and Sysoev O. (2005). Monotonic regression for the detection of temporal trends in environmental quality data. Communications in Mathematical and in Computer Chemistry Vol. 54, 535-550.

[19] IPCC (2007). Nobel Peace Prize, Intergovernmental Panel on Climate Change Facts. Nobelprize.org. Nobel Media AB 2014. Web. 12 Dec 2014. http://www . nobelprize.org/nobel_prizes/peace/laureates/2007/ipcc-facts.html

[20] Jones, P. D., and Mann, M. E. (2004). Climate over past millennia. Reviews of Geophysics, Vol. 42) RG2002

[21] Mammen, E. (1991). Estimating a Smooth Monotone Regression Function. The Annals of Statistics Vol 19 724-740. 
[22] Meal, D. (2011). Statistical Analysis for Monotone Trend. The NCSU Water Quality Group Newsletter Issue 135 1-11

[23] Memendez, P.; Ghosh, S.; Hans, R. K. and Tinner, W. (2013). On trend estimation under monotone Gaussian subordination with long-memory: application to fossil pollen series. Journal of Nonparametric Statistics Vol 25 765-785

[24] Moulines, E., Roueff, F., and Taqqu, M. S. (2008). A wavelet whittle estimator of the memory parameter of a nonstationary Gaussian time series. The Annals of Statistics, 1925-1956.

[25] Mukherjee, H. (1988). Monotone Nonparametric Regression. The Annals of Statistics Vol 16 741-750.

[26] NAAQS (1990). National Ambient Air Quality Standards (NAAQS), Amendment to the "Clean Air Act" http://www.epa.gov/air/criteria.html.

[27] NASA GISS Surface Temperature Analysis http://data.giss.nasa.gov/gistemp/graphs_v3/

[28] Pal, J. and Woodroofe, M. (2007). Large Sample Properties of Shape Restricted Regression Estimators With Smoothness Adjustments. Statistics Sinica, Vol. 17, 1601-1616.

[29] Peligrad, M. and Utev, S. (2005). A new maximal inequality and invariance principle for stationary sequences. The Annals of Probability Vol. 33, 798-815.

[30] Ramsay, J.O.(1998). Estimating Smooth Monotone Functions. Journal of the Royal Society: Series B Vol. 60, 365-375.

[31] Rao, P.B.L.S.(1969). Estimation of unimodal density. Sankhya Ser. A Vol 31, 23-36.

[32] Resnick, S. I.(1999). A Probability Path. Birkhäuser, Boston.

[33] Robinson, P. M. (1997). Large-sample inference for nonparametric regression with dependent errors. The Annals of Statistics, Vol. 25 no. 5, 2054-2083.

[34] Robertson, T., Wright, F. T. and Dykstra, R. L.(1988). Order Restricted Statistical Inference. New York: Wiley.

[35] Robinson, P. M. (2009) Inference on nonparametrically trending time series with fractional errors. Econometric Theory, Vol. 25, no. 6, 1716-1733.

[36] Rockafellar, R.T.(1970). Convex Analysis. Princeton University Press, Princeton.

[37] Samorodnitsky, G (2006). Long range dependence. Foundations and Trends ${ }^{\circledR}$ in Stochastic Systems, Vol. 1, No. 3, 163-257.

[38] Steig, E., Schneider, D., Rutherford, S., Mann, M., Comiso, J., and Shindell, D. (2009). Warming of the Antarctic ice-sheet surface since the 1957 International Geophysical Year. Nature Vol. 457, 459-463. 
[39] Stoev, S. A., Taqqu, M. S., Park, C., Marron, J. S. (2005). On the wavelet spectrum diagnostic for Hurst parameter estimation in the analysis of Internet traffic. Computer Networks, 48, 423-445.

[40] Sun, J. and Woodroofe, M.(1996) Adaptive smoothing for a penalized NPMLE of a non-increasing density. Journal of Statistical Planning and Inference Vol. 52, 143-159.

[41] Taqqu, M. S. (1975). Weak convergence to fractional Brownian motion and to Rosenblatt process. Z. Wahrsch. Verw. Gebiete Vol. 31, 287-302.

[42] Taqqu, M. S. (1979). Convergence of integrated processes of arbitrary Hermite rank. Z. Wahrsch. Verw. Gebiete Vol. 50, 53-80.

[43] Willinger, W., Taqqu, M.S., Leland, W. E., and Wilson, V. (1995). Self-Similarity in high-speed packet traffic: analysis and modeling of Ethernet traffic measurements. Statistical Science, 10, 67-85.

[44] Woodroofe, M. and Sun, J. (1993) A penalized maximum likelihood estimate of $f(0+)$ when $f$ is non-uncreasing. Statistica Sinica Vol. 3, 501-515.

[45] Wu, W. B., Woodroofe, M. and Mentz, G. (2001) Isotonic regression: another look at the change point problem. Biometrika Vol. 88, 793-804.

[46] Wu, W.B. and Zhao, Z. (2007). Inference of trends in time series. J. R. Stat. Soc. Ser. B, Vol. 69, no. 3, 391-410.

[47] Zhao, O. and Woodroofe, M.(2012) Estimating a monotone trend. Statistica Sinica, Vol. 22, 359-378. 


\title{
Supplement to Inference for Monotone Trends Under Dependence
}

\author{
Pramita Bagchi, Moulinath Banerjee, and Stilian A. Stoev
}

July 2, 2018

\begin{abstract}
Here we discuss the auxiliary results related to the main paper and provide some technical proofs of lemmas mentioned in the paper. We also provide some details on our simulation scheme and other simulation results which do not appear in the paper for space constraint.
\end{abstract}

\section{Tables and Figures}

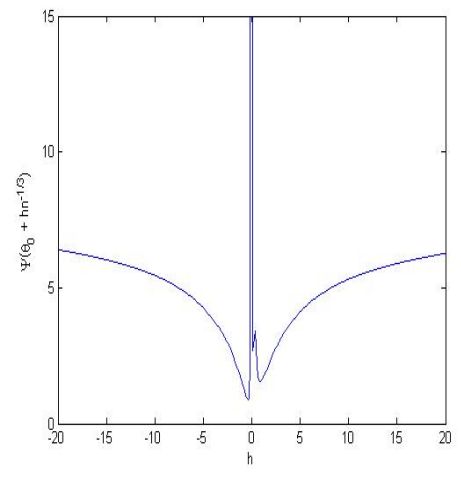

(a) Short Range Dependence

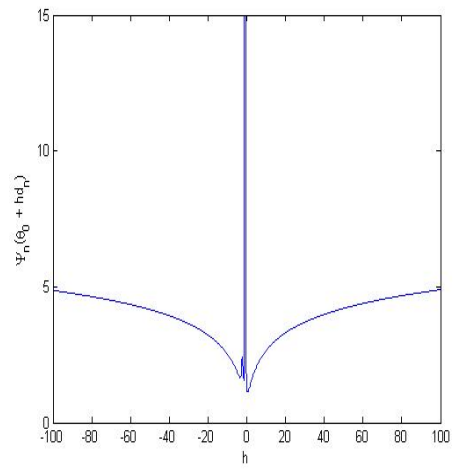

(b) Long Range Dependence

Figure 1: Shape of $\Psi$-Statistic as a function of $h$

\section{Proof of Results of Section 4}

In this section we present the proofs of the Results discussed in Section 4. First note that the isotonic regression estimator can be represented in terms 
Table 1: Confidence Intervals with $\operatorname{ARMA}(2,2), \operatorname{AR}$ coeffs $0.8,-0.5$ and MA coeffs $-0.2,0.3$

\begin{tabular}{|c|c|c|c|c|c|c|c|c|c|c|c|c|c|c|c|c|}
\hline \multirow{3}{*}{$\mathrm{n}$} & \multicolumn{8}{|c|}{$m_{1}(t)$} & \multicolumn{8}{|c|}{$m_{2}(t)$} \\
\hline & \multicolumn{2}{|c|}{$L_{n}$} & \multicolumn{2}{|c|}{$\Psi_{n}$} & \multicolumn{2}{|c|}{ IRE1 $^{1}$} & \multicolumn{2}{|c|}{ IRE2 $^{2}$} & \multicolumn{2}{|c|}{$L_{n}$} & \multicolumn{2}{|c|}{$\Psi_{n}$} & \multicolumn{2}{|c|}{ IRE1 } & \multicolumn{2}{|c|}{ IRE2 } \\
\hline & Cov. & Len. & Cov. & Len. & Cov. & Len. & Cov. & Len. & Cov. & Len. & Cov. & Len. & Cov. & Len. & Cov. & Len. \\
\hline 100 & 89.1 & 0.443 & 90.8 & 0.537 & 86.5 & 0.504 & 87.3 & 0.511 & 88.3 & 0.407 & 89.9 & 0.523 & 81.8 & 0.485 & 80.1 & 0.478 \\
\hline 200 & 89.7 & 0.352 & 92 & 0.392 & 85.8 & 0.413 & 84.9 & 0.433 & 87.6 & 0 . & 89.8 & 0. & 78.6 & 0.399 & 79.2 & 0.401 \\
\hline 500 & 89.3 & 0.261 & 91.4 & 0.307 & 84.9 & 0.312 & 85.7 & 0.332 & 88.9 & 0.230 & 90.4 & 0.312 & 79.9 & 0.298 & 73.4 & 0.225 \\
\hline 1000 & 90.2 & 0.208 & 90.7 & 0.262 & 85.9 & 0.257 & 86.8 & 0.239 & 85.9 & 0.180 & 90.2 & 0.279 & 76.9 & 0.215 & 80.2 & 0.219 \\
\hline 2000 & 91 & 0.163 & 90.9 & 0.205 & 86.8 & 0.209 & 85.5 & 0.211 & 90.1 & 0.141 & 90.8 & 0.211 & 80.1 & 0.199 & 75.5 & 0.186 \\
\hline 5000 & 89.1 & 0.121 & 91.7 & 0.169 & 89.9 & 0.169 & 88.7 & 0.178 & 90.2 & 0.105 & 90.5 & 0.134 & 81.5 & 0.114 & 81.4 & 0.112 \\
\hline
\end{tabular}

Table 2: Confidence Intervals with $\mathrm{AR}(2)$ and AR coeffs $0.95,0.8$

\begin{tabular}{|c|c|c|c|c|c|c|c|c|c|c|c|c|c|c|c|c|}
\hline \multirow{3}{*}{$\mathrm{n}$} & \multicolumn{8}{|c|}{$m_{1}(t)$} & \multicolumn{8}{|c|}{$m_{2}(t)$} \\
\hline & \multicolumn{2}{|c|}{$L_{n}$} & \multicolumn{2}{|c|}{$\Psi_{n}$} & \multicolumn{2}{|c|}{ IRE1 $^{3}$} & \multicolumn{2}{|c|}{ IRE2 $^{4}$} & \multicolumn{2}{|c|}{$L_{n}$} & \multicolumn{2}{|c|}{$\Psi_{n}$} & \multicolumn{2}{|c|}{ IRE1 } & \multicolumn{2}{|c|}{ IRE2 } \\
\hline & Cov. & Len. & Cov. & Len. & Cov. & Len. & Cov. & Len. & Cov. & Len. & Cov. & Len. & Cov. & Len. & Cov. & Len. \\
\hline 100 & 87.4 & 1.006 & 90.8 & 1.783 & 84.2 & 1.558 & 85.7 & 1.679 & 83.3 & 0.879 & 89.7 & 1.003 & 68.9 & 0.901 & 73.5 & 1.001 \\
\hline 200 & 88.9 & 814 & 90.5 & 1.498 & 82.7 & 1.293 & 85.5 & 1.311 & 82.4 & 0.775 & 89.8 & 0.892 & 77.5 & 0.865 & 74.7 & 0.828 \\
\hline 500 & 89.2 & 573 & 91.1 & 1.134 & 83.8 & 1.020 & 81.2 & 1.001 & 88.5 & 0.499 & 90.3 & 0.687 & 70.3 & 0.662 & 73.1 & 0.698 \\
\hline 1000 & 89.1 & 0.458 & 91.6 & 0.827 & 85.9 & 0.809 & 83.6 & 0.798 & 89.9 & 0.387 & 91.6 & 0.568 & 72.4 & 0.525 & 71.9 & 0.517 \\
\hline 2000 & 90.3 & 0.357 & 92.3 & 0.689 & 86.5 & 0.715 & 81.5 & 0.687 & 87.8 & 0.296 & 90.8 & 0.499 & 75.6 & 0.468 & 72.3 & 0.455 \\
\hline 5000 & 89.3 & 0.283 & 91.4 & 0.514 & 83.5 & 0.592 & 82.9 & 0.527 & 89.5 & 0.198 & 91.3 & 0.401 & 76.9 & 0.379 & 77.8 & 0.400 \\
\hline
\end{tabular}

Table 3: Confidence Intervals with fractional Gaussian noise, $\mathrm{H}=0.9$

\begin{tabular}{|c|c|c|c|c|c|c|c|c|c|c|c|c|}
\hline \multirow{3}{*}{$\mathrm{n}$} & \multicolumn{6}{|c|}{$m_{1}(t)$} & \multicolumn{6}{|c|}{$m_{2}(t)$} \\
\hline & \multicolumn{2}{|c|}{$L_{n}$} & \multicolumn{2}{|c|}{$\Psi_{n}$} & \multicolumn{2}{|c|}{ IRE } & \multicolumn{2}{|c|}{$L_{n}$} & \multicolumn{2}{|c|}{$\Psi_{n}$} & \multicolumn{2}{|c|}{ IRE } \\
\hline & Cov. & Len. & Cov. & Len. & Cov. & Len. & Cov. & Len. & Cov. & Len. & Cov. & Len. \\
\hline 100 & 84.5 & 0.662 & 90.4 & 0.892 & 89.1 & 0.659 & 81.7 & 0.688 & 87.6 & 0.902 & 71.2 & 0.723 \\
\hline 200 & 81.5 & 0.602 & 89.6 & 0.804 & 73.2 & 0.600 & 77.8 & 0.615 & 83.9 & 0.823 & 72.3 & 0.667 \\
\hline 500 & 71.6 & 0.558 & 90.4 & 0.741 & 81.7 & 0.550 & 78.5 & 0.587 & 88.7 & 0.774 & 66.3 & 0.597 \\
\hline 1000 & 72.2 & 0.503 & 92.1 & 0.691 & 75.8 & 0.500 & 73.9 & 0.514 & 89.5 & 0.712 & 71.8 & 0.561 \\
\hline 2000 & 83.1 & 0.478 & 91.1 & 0.658 & 79.2 & 0.470 & 80.1 & 0.495 & 89.9 & 0.675 & 73.5 & 0.518 \\
\hline 5000 & 81.7 & 0.434 & 91.8 & 0.597 & 76.7 & 0.432 & 81.2 & 0.462 & 89.7 & 0.613 & 74.9 & 0.499 \\
\hline
\end{tabular}

Table 4: Confidence Intervals with fractional Gaussian noise, $\mathrm{H}=0.99$

\begin{tabular}{|c|c|c|c|c|c|c|c|c|c|c|c|c|}
\hline \multirow{3}{*}{$\mathrm{n}$} & \multicolumn{6}{|c|}{$m_{1}(t)$} & \multicolumn{6}{|c|}{$m_{2}(t)$} \\
\hline & \multicolumn{2}{|c|}{$L_{n}$} & \multicolumn{2}{|c|}{$\Psi_{n}$} & \multicolumn{2}{|c|}{ IRE } & \multicolumn{2}{|c|}{$L_{n}$} & \multicolumn{2}{|c|}{$\Psi_{n}$} & \multicolumn{2}{|c|}{ IRE } \\
\hline & Cov. & Len. & Cov. & Len. & Cov. & Len. & Cov. & Len. & Cov. & Len. & Cov. & Len. \\
\hline 100 & 80.2 & 0.795 & 87.8 & 0.912 & 71.2 & 0.801 & 75.4 & 0.812 & 84.7 & 0.921 & 70.2 & 0.872 \\
\hline 200 & 71.3 & 0.742 & 88.4 & 0.864 & 81.9 & 0.751 & 72.1 & 0.798 & 83.1 & 0.901 & 68.4 & 0.805 \\
\hline 500 & 70.6 & 0.697 & 89.1 & 0.831 & 70.2 & 0.701 & 70.8 & 0.745 & 85.3 & 0.858 & 65.5 & 0.763 \\
\hline 1000 & 81.5 & 0.654 & 88.9 & 0.814 & 70.8 & 0.660 & 71.1 & 0.687 & 86.2 & 0.832 & 63.9 & 0.705 \\
\hline 2000 & 68.8 & 0.631 & 89.1 & 0.803 & 81.8 & 0.638 & 70.5 & 0.651 & 85.8 & 0.816 & 71.4 & 0.674 \\
\hline 5000 & 73.7 & 0.593 & 89.9 & 0.785 & 71.2 & 0.601 & 72.7 & 0.612 & 88.9 & 0.773 & 72.6 & 0.655 \\
\hline
\end{tabular}


Table 5: Confidence Intervals with $\operatorname{FARIMA}(2,0.2,1)$ errors, AR coeffs 0.5,0.5 ; MA coeff $0.6, \mathrm{H}=0.7$

\begin{tabular}{|c|c|c|c|c|c|c|c|c|c|c|c|c|}
\hline \multirow{3}{*}{$\mathrm{n}$} & \multicolumn{6}{|c|}{$m_{1}(t)$} & \multicolumn{6}{|c|}{$m_{2}(t)$} \\
\hline & \multicolumn{2}{|c|}{$L_{n}$} & \multicolumn{2}{|c|}{$\Psi_{n}$} & \multicolumn{2}{|c|}{ IRE } & \multicolumn{2}{|c|}{$L_{n}$} & \multicolumn{2}{|c|}{$\Psi_{n}$} & \multicolumn{2}{|c|}{ IRE } \\
\hline & Cov. & Len. & Cov. & Len. & Cov. & Len. & Cov. & Len. & Cov. & Len. & Cov. & Len. \\
\hline 100 & 82.1 & 0.599 & 89.1 & 0.986 & 86.3 & 0.601 & 77.5 & 0.667 & 89.2 & 0.979 & 60.1 & 0.754 \\
\hline 200 & 81.9 & 0.520 & 89.9 & 0.888 & 84.8 & 0.521 & 78.2 & 0.589 & 88.7 & 0.876 & 62.5 & 0.682 \\
\hline 500 & $\begin{array}{l}79.8 \\
\end{array}$ & 0.432 & 90.3 & 0.747 & 81.2 & 0.439 & 71.6 & 0.495 & 91.1 & 0.735 & 74.8 & 0.595 \\
\hline 1000 & 80.4 & 0.362 & 91.2 & 0.632 & 79.8 & 0.370 & 72.8 & 0.401 & 90.1 & 0.609 & 71.1 & 0.502 \\
\hline 2000 & 77.6 & 0.311 & 90.9 & 0.546 & 89.2 & 0.318 & 74.1 & 0.362 & 90.4 & 0.515 & 72.9 & 0.468 \\
\hline 5000 & 81.7 & 0.262 & 91.5 & 0.446 & 81.2 & 0.273 & 77.5 & 0.298 & 90.8 & 0.407 & 72.6 & 0.375 \\
\hline
\end{tabular}

Table 6: Confidence Intervals for $m_{1}(0.5)$ in the presence of FGN errors and the FARIMA process in Table 5 using estimated $H$

\begin{tabular}{|c|c|c|c|c|c|c|c|c|}
\hline \multirow{2}{*}{$\mathrm{n}$} & \multicolumn{2}{|c|}{ FGn, $\mathrm{H}=0.6$} & \multicolumn{2}{|c|}{ FGn, $\mathrm{H}=0.8$} & \multicolumn{2}{|c|}{ FGn, $\mathrm{H}=0.9$} & \multicolumn{2}{c|}{ FARIMA, $\mathrm{H}=0.7$} \\
\cline { 2 - 10 } & Cov. & Len. & Cov. & Len. & Cov. & Len. & Cov. & Len. \\
\hline 100 & 90.1 & 0.643 & 90.8 & 0.737 & 90.5 & 0.904 & 89.3 & 0.607 \\
\hline 200 & 89.7 & 0.552 & 92 & 0.592 & 89.8 & 0.813 & 88.6 & 0.512 \\
\hline 500 & 91.3 & 0.461 & 91.4 & 0.407 & 88.9 & 0.712 & 89.9 & 0.430 \\
\hline 1000 & 90.2 & 0.408 & 90.7 & 0.362 & 89.9 & 0.657 & 90.9 & 0.380 \\
\hline 2000 & 91 & 0.363 & 90.9 & 0.305 & 90.1 & 0.509 & 90.1 & 0.341 \\
\hline 5000 & 89.1 & 0.221 & 91.7 & 0.269 & 89.9 & 0.369 & 90.2 & 0.205 \\
\hline
\end{tabular}


of the partial sum process $U_{n}$ defined as in the main paper.

Proposition 2.1. We have

$$
\begin{aligned}
\hat{m}_{n}(t)= & \mathcal{L} \circ \mathcal{T}_{(0,1]}\left(U_{n}\right)(t) \\
\hat{m}_{n}^{0}(t)= & \left(\mathcal{L} \circ \mathcal{T}_{\left(0, t_{l}\right]}\left(U_{n}\right)(t) \wedge \theta_{0}\right) \mathbf{1}_{\left(0, t_{l}\right]}(t) \\
& +\theta_{0} \mathbf{1}_{\left(t_{l}, t_{0}\right]}(t)+\left(\mathcal{L} \circ \mathcal{T}_{\left(t_{l}, 1\right]}\left(U_{n}\right)(t) \vee \theta_{0}\right) \mathbf{1}_{\left(t_{0}, 1\right]}(t) .
\end{aligned}
$$

This representation follows from Chapter 2 of Robertson et. al. (1988)[9] in the case of $\hat{m}_{n}$, and from Section 2 of Banerjee and Wellner (2001)[4], in the case of $\hat{m}_{n}^{0}$.

\subsection{The Process $\mathbb{V}_{n}$}

Proof of Theorem A.1: It is enough to show that for all $c>0$, we have $\left.\left.\mathbb{V}_{n}\right|_{[-c, c]} \Rightarrow \mathbb{G}\right|_{[-c, c]}$ in $C([-c, c])$ equipped with the uniform norm. Fix $c>0$ and note that since $\left(a_{n}, b_{n}\right] \uparrow \mathbb{R}$, as $n \rightarrow \infty$, without loss of generality we may assume that $[-c, c] \subset\left(a_{n}, b_{n}\right]$. Write $\mathbb{V}_{n}(z)=\mathbb{W}_{n}(z)+\Lambda_{n}(z), z \in[-c, c]$, where

$$
\mathbb{W}_{n}(z):=d_{n}^{-2} n^{-1}\left(v_{n}\left(t_{0}+z d_{n}\right)-v_{n}\left(t_{0}\right)\right),
$$

with $v_{n}(t)=\sum_{i=1}^{\lfloor n t\rfloor} \epsilon_{i}+(n t-\lfloor n t\rfloor) \epsilon_{\lfloor n t\rfloor+1}$. Then,

$$
\Lambda_{n}(z)=\Upsilon_{n}(z)+\mathbf{R}_{n}(z)
$$

where $\Upsilon_{n}(z)=d_{n}^{-2}\left[M\left(t_{0}+d_{n} z\right)-M\left(t_{0}\right)-m\left(t_{0}\right) d_{n} z\right]$, and $\mathbf{R}_{n}(z)=d_{n}^{-2}\left[\left(M_{n}-\right.\right.$ $\left.M)\left(t_{0}+d_{n} z\right)-\left(M_{n}-M\right)\left(t_{0}\right)\right]$. Hence we have

$$
\sup _{z \in\left(a_{n}, b_{n}\right]}\left|\mathbf{R}_{n}(z)\right| \leq 2 d_{n}^{-2} \sup _{0 \leq t \leq 1}\left|M_{n}(t)-M(t)\right|=O\left(d_{n}^{-2} n^{-1}\right) .
$$

The latter vanishes as $n \rightarrow \infty$ because,

$$
d_{n}^{-2} n^{-1}= \begin{cases}n^{-\frac{1}{3}} & \text { under weak dependence } \\ n^{-\frac{2-d}{2+d}} & \text { under strong dependence. }\end{cases}
$$

Thus, the remainder term $\mathbf{R}_{n}$ in (2) can be neglected and a Taylor series expansion of the deterministic function $M$ at $t_{0}$ in the term $\Upsilon_{n}$ yields,

$$
\Lambda_{n}(z) \rightarrow \frac{1}{2} m^{\prime}\left(t_{0}\right) z^{2}
$$

as $n \rightarrow \infty$ uniformly on $[-c, c]$. 
Now, we deal with the term $\mathbb{W}_{n}$. By the stationarity of $\left\{\epsilon_{i}\right\}_{i \in \mathbb{Z}}$, we have

$$
\left\{v_{n}\left(t_{0}+z d_{n}\right)-v_{n}\left(t_{0}\right)\right\}_{z \in \mathbb{R}} \stackrel{d}{=}\left\{v_{n}\left(z d_{n}\right)\right\}_{z \in \mathbb{R}}=\left\{v_{\hat{n}}(z)\right\}_{z \in \mathbb{R}},
$$

where $\widehat{n}:=n d_{n}$. Note that $\widehat{n}$ may not be an integer. The definition of $v_{n}$ makes sense even if $n$ is not an integer. For rest of the proof we will use other sequences indexed by $\widehat{n}$ and we define $\sigma_{\widehat{n}}=\sigma_{\lfloor\widehat{n}\rfloor}$ and $w_{\widehat{n}}(t)=v_{\widehat{n}}(t) / \sigma_{\widehat{n}}($ recall (5) from the main paper). With this convention our following arguments remain valid at least asymptotically if $\widehat{n} \rightarrow \infty$ as $n \rightarrow \infty$.

Next we can write,

$$
\left\{\mathbb{W}_{n}(z)\right\}_{z \in \mathbb{R}} \stackrel{d}{=}\left\{d_{n}^{-2} n^{-1} v_{\widehat{n}}(z)\right\}_{z \in \mathbb{R}}=\left\{d_{n}^{-2} n^{-1} \sigma_{\widehat{n}} w_{\widehat{n}}(z)\right\}_{z \in \mathbb{R}},
$$

where $w_{n}$ is as in (5) of the main paper. Observe that under both short- and long-range dependence assumptions, we have $\left.\left.w_{\widehat{n}}\right|_{[-c, c]} \Rightarrow \mathbb{W}\right|_{[-c, c]}$, as $\widehat{n} \rightarrow \infty$ in the Skorokhod $J_{1}$-topology, where $\mathbb{W}$ denotes either the two-sided Brownian motion or the process $B_{r, H}$ (recall Section 3 of the main paper). Since the limit processes (in both cases) have versions with continuous paths, the $J_{1}$-convergence implies also convergence in the uniform topology. To complete the proof, it remains to show that $\widehat{n} \rightarrow \infty$ in both cases with the appropriate choice of $d_{n}$ and constants.

(i) Under short-range dependence, with $d_{n}=n^{-\frac{1}{3}}$, we have $\hat{n} \equiv n d_{n} \rightarrow$ $\infty$ and, by (8) from the main paper, $d_{n}^{-2} n^{-1} \sigma_{\hat{n}} \rightarrow \tau$ as $n \rightarrow \infty$, which yields $a=\tau$.

(ii) Under long-range dependence, we want $d_{n}$ such that $d_{n}^{-2} n^{-1} \sigma_{\hat{n}} \rightarrow\left|\eta_{1}\right|$ as $n \rightarrow \infty$ where $\eta_{1}$ is the Hermite rank. By relation (10) of the main paper this is equivalent to

$$
\begin{aligned}
\left|\eta_{1}\right| & =d_{n}^{-2} n^{-1}\left|\eta_{1}\right|\left(n d_{n}\right)^{1-\frac{d}{2}} l_{1}\left(n d_{n}\right)^{\frac{1}{2}} \\
& \Longleftrightarrow d_{n}^{1+\frac{d}{2}}=n^{-\frac{d}{2}} l_{1}\left(n d_{n}\right)^{\frac{1}{2}} \\
& \Longleftrightarrow d_{n}=n^{-\frac{d}{2+d}} l_{2}(n),
\end{aligned}
$$

where $l_{2}$ is another slowly varying function at infinity. This choice of $d_{n}$ ensures that $\hat{n} \equiv n d_{n} \rightarrow \infty$ as $n \rightarrow \infty$ and $a=\left|\eta_{1}\right|$, by (9) of the main paper. This completes the proof.

\subsection{The Processes $X_{n}$ and $Y_{n}$}

The processes $\mathbb{V}_{n}(z), X_{n}(z)$ and $Y_{n}(z)$ are only defined for $z \in\left(a_{n}, b_{n}\right]$. Ultimately, we have that $\left(a_{n}, b_{n}\right] \uparrow \mathbb{R}$. For technical convenience, however, 
we shall extend the definitions of these processes to the entire real line. This is best done by extending $\mathbb{V}_{n}$ in such a way that Relations (27) mentioned in the main paper continue to hold for all $z \in(-\infty, \infty)$. To this end, let

$$
\mathbb{V}_{n}(z):= \begin{cases}\mathbb{V}_{n}(z) & , z \in\left(a_{n}, b_{n}\right] \\ \lambda_{\ell}\left(z-a_{n}\right)+\mathbb{V}_{n}\left(a_{n}+\right) & , z \in\left(-\infty, a_{n}\right] \\ \lambda_{r}\left(z-b_{n}\right)+\mathbb{V}_{n}\left(b_{n}\right) & , z \in\left(b_{n}, \infty\right),\end{cases}
$$

where $\lambda_{\ell}=\lim _{z \downarrow a_{n}} \mathcal{L} \circ \mathcal{T}_{\left(a_{n}, b_{n}\right]}\left(\mathbb{V}_{n}\right)(z)$ and $\lambda_{r}=\mathcal{L} \circ \mathcal{T}_{\left(a_{n}, b_{n}\right]}\left(\mathbb{V}_{n}\right)\left(b_{n}\right)$. That is, $\lambda_{\ell}$ and $\lambda_{r}$ may be viewed as the smallest and largest left slopes of the GCM of $\mathbb{V}_{n}$ over the interval $\left(a_{n}, b_{n}\right]$.

The so-defined extension of $\mathbb{V}_{n}$ has the following important property:

$$
\mathcal{T}_{(-\infty, \infty)}\left(\mathbb{V}_{n}\right)(z)=\mathcal{T}_{\left(a_{n}, b_{n}\right]}\left(\mathbb{V}_{n}\right)(z), \quad \text { for all } z \in\left(a_{n}, b_{n}\right],
$$

and in fact $\mathcal{T}_{(-\infty, c]}\left(\mathbb{V}_{n}\right)(z)=\mathcal{T}_{\left(a_{n}, c\right]}\left(\mathbb{V}_{n}\right)(z), z \in\left(a_{n}, c\right]$ and $\mathcal{T}_{(c, \infty)}\left(\mathbb{V}_{n}\right)(z)=$ $\mathcal{T}_{\left(c, b_{n}\right]}\left(\mathbb{V}_{n}\right)(z), z \in\left(c, b_{n}\right]$. This shows that Relations (27) of the main paper continue to hold and in fact $a_{n}$ and $b_{n}$ therein can be replaced by $-\infty$ and $\infty$, respectively. Therefore, from now on, we shall consider the processes $X_{n}=\left\{X_{n}(z)\right\}_{z \in \mathbb{R}}$ and $Y_{n}=\left\{Y_{n}(z)\right\}_{z \in \mathbb{R}}$, defined as follows

$$
\begin{aligned}
X_{n}(z)= & \mathcal{L} \circ \mathcal{T}_{(-\infty, \infty)}\left(\mathbb{V}_{n}\right)(z) \\
Y_{n}(z)= & \left(\mathcal{L} \circ \mathcal{T}_{\left(-\infty, l_{n}\right]}\left(\mathbb{V}_{n}\right)(z) \wedge 0\right) \mathbf{1}_{\left(-\infty, l_{n}\right]}(z)+0 \times \mathbf{1}_{\left(l_{n}, 0\right]}(z) \\
& +\left(\mathcal{L} \circ \mathcal{T}_{\left(l_{n}, \infty\right]}\left(\mathbb{V}_{n}\right)(z) \vee 0\right) \mathbf{1}_{(0, \infty)}(z) .
\end{aligned}
$$

The paths of the processes $X_{n}$ and $Y_{n}$ are left-continuous non-decreasing step-functions, which are constant on $\left(-\infty, a_{n}\right]$ and $\left(b_{n}, \infty\right)$. As argued above, over $\left(a_{n}, b_{n}\right]$ they are given by $(21)$ of the main paper.

For the next step we need the following result from $\mathrm{AH}[2]$ :

Theorem 2.1 (Adapted from AH[2]). Consider a sequence of stochastic processes $\left\{V_{n}(z)\right\}_{z \in \mathbb{R}}, n=1,2, \cdots$ with paths in $C(\mathbb{R})$. Assume that

(1) (Compact boundedness) For every compact set $K$ and $\delta>0$, there is a finite $M=M(K, \delta)$ such that

$$
\limsup _{n \rightarrow \infty} \mathbb{P}\left(\sup _{z \in K}\left|V_{n}(z)\right|>M\right)<\delta
$$

(2) (Lower bound) For every $\delta>0$, there are finite $0<\tau=\tau(\delta)$ and $0<\kappa=\kappa(\delta)$ such that

$$
\liminf _{n \rightarrow \infty} \mathbb{P}\left(\inf _{|z| \geq \tau}\left(V_{n}(z)-\kappa|z|\right)>0\right)>1-\delta
$$


(3) (Small downdippings) Given $\epsilon, \delta, \tilde{\tau}>0$,

$$
\begin{array}{r}
\limsup _{n \rightarrow \infty} \mathbb{P}\left(\inf _{\tilde{\tau} \leq z \leq c} \frac{V_{n}(z)}{z}-\inf _{\tilde{\tau} \leq z} \frac{V_{n}(z)}{z}>\epsilon\right)<\delta \\
\limsup _{n \rightarrow \infty} \mathbb{P}\left(\inf _{z \leq-\tilde{\tau}} \frac{V_{n}(z)}{z}-\inf _{-c \leq z \leq-\tilde{\tau}} \frac{V_{n}(z)}{z}<-\epsilon\right)<\delta
\end{array}
$$

for all large enough $c>0$

Then for any finite interval $I$ in $\mathbb{R}$ and $\epsilon>0$,

$$
\lim _{c \rightarrow \infty} \limsup _{n \rightarrow \infty} \mathbb{P}\left(\sup _{I}\left|\mathcal{T}_{[-c, c]}\left(V_{n}\right)(.)-\mathcal{T}\left(V_{n}\right)(.)\right|>\epsilon\right)=0 .
$$

This also holds true if we replace $\mathcal{T}$ by $\mathcal{T}_{O}$ for any interval $O \subseteq \mathbb{R}$ or $\mathcal{T}_{O_{n}}$ where $O_{n}$ is a sequence of intervals such that $O_{n} \uparrow O$, with $O \subseteq \mathbb{R}$. In these cases $\mathcal{T}_{[-c, c]}$ in (11) is replaced by $\mathcal{T}_{K_{c}}$, for some sequence of compact intervals $K_{c}$ such that $K_{c} \uparrow O$ as $c \rightarrow \infty$.

Proposition 2.2. The processes $V_{n}:=\mathbb{V}_{n}$ in (26) (main paper) satisfy the conditions of Theorem 2.1.

Proof. For the process $\mathbb{V}_{n},(7)$ is satisfied by TheoremA.1. Also, by Proposition 1 from $\mathrm{AH}[2],(8),(9)$ and (10) are implied by (3) and the following:

Assume that for any $\epsilon, \delta>0$, there exist $\kappa=\kappa(\epsilon, \delta)>0$ and $\tau=$ $\tau(\epsilon, \delta)>0$ such that

$$
\sup _{n} \mathbb{P}\left(\sup _{|z| \geq \tau} \frac{\mathbb{W}_{n}(z)}{\kappa|z|}>\epsilon\right)<\delta
$$

This is implied by Lemma 2.1 stated below and (4) imply (12). So all the conditions of Theorem 2.1 are satisfied.

Lemma 2.1. For each $\epsilon, \delta, \kappa>0$, there exist $\tau=\tau(\epsilon, \delta, \kappa)>0$ and $m_{0}=$ $m_{0}(\epsilon, \delta, \kappa)<\infty$ such that

$$
\sup _{n \geq m_{0}} \mathbb{P}\left(\sup _{|s| \geq \tau} \frac{\left|w_{\hat{n}}(z)\right|}{\kappa|z|}>\epsilon\right)<\delta
$$

Proof. The proof of lemma for long range dependent errors is already done in $\mathrm{AH}[2]$ (page 1921-1922). In the short range dependence case we have to verify

$$
\mathbb{P}\left(\max _{1 \leq k \leq \tilde{n}}\left|S_{k}\right|>\lambda \sigma_{\tilde{n}}\right) \leq \frac{C}{\lambda^{2}}
$$


under assumption of weak dependence, where $\tilde{n}=\hat{n} \Delta_{i}$ and $\Delta_{i}$ is a constant.

But, by Chebyshev's inequality and relations (7) and (8) in the main paper above we have,

$$
\begin{aligned}
\mathbb{P}\left(\max _{1 \leq k \leq \tilde{n}}\left|S_{k}\right|>\lambda \sigma_{\tilde{n}}\right) & \leq \frac{\mathbb{E}\left(\max _{1 \leq k \leq \tilde{n}} S_{k}^{2}\right)}{\lambda^{2} \sigma_{\tilde{n}}^{2}} \\
& \leq \frac{6\left[\sigma^{2}+\Gamma\right] \tilde{n}}{\lambda^{2} \sigma_{\tilde{n}}^{2}} \\
& \leq \frac{C}{\lambda^{2}} .
\end{aligned}
$$

So (13) is satisfied in this case. This proves the Lemma.

This result will be used to "localize" certain continuous mapping arguments to a compact interval.

Proof of Proposition A.1: By adding and subtracting $\theta_{0}$ and expanding the squares in the two sums in Relation (4) in main paper, we obtain

$$
\begin{aligned}
L_{n}= & \frac{n}{\sigma_{n}^{2}}(-\underbrace{2 \sum_{i=1}^{n}\left(Y_{i}-\theta_{0}\right)\left(\hat{m}_{n}^{0}\left(t_{i}\right)-\theta_{0}\right)}_{=: A_{0}}+\underbrace{\sum_{i=1}^{n}\left(\hat{m}_{n}^{0}\left(t_{i}\right)-\theta_{0}\right)^{2}}_{=: B_{0}}) \\
& -\frac{n}{\sigma_{n}^{2}}(\underbrace{-2 \sum_{i=1}^{n}\left(Y_{i}-\theta_{0}\right)\left(\hat{m}_{n}\left(t_{i}\right)-\theta_{0}\right)}_{=: A}+\underbrace{\left.\sum_{i=1}^{n}\left(\hat{m}_{n}\left(t_{i}\right)-\theta_{0}\right)^{2}\right)}_{=: B} .
\end{aligned}
$$

It is known by the so-called pooled adjacent violators (PAV) characterization of isotonic regression that $\hat{m}_{n}\left(t_{i}\right) \mathrm{s}$ are sample averages of $Y_{j}$ s over nonoverlapping blocks of indices $j$. (see Brunk (1970)[7]). This is also true for $\hat{m}_{n}^{0}\left(t_{i}\right)$ s whenever $\hat{m}_{n}^{0}\left(t_{i}\right) \neq \theta_{0}$. Therefore, by grouping together the terms in the sum $A$ that correspond to the same $\hat{m}_{n}\left(t_{i}\right)$ s, we obtain that $A=2 B$. Similarly, we have $A_{0}=2 B_{0}$ and therefore,

$$
L_{n}=\frac{n}{\sigma_{n}^{2}}\left(-B_{0}+B\right)=\frac{n}{\sigma_{n}^{2}}\left(\sum_{i=1}^{n}\left(\hat{m}_{n}\left(t_{i}\right)-\theta_{0}\right)^{2}-\sum_{i=1}^{n}\left(\hat{m}_{n}^{0}\left(t_{i}\right)-\theta_{0}\right)^{2}\right) .
$$

Recall now that $X_{n}(z)=d_{n}^{-1}\left(\hat{m}_{n}\left(t_{0}+d_{n} z\right)-\theta_{0}\right)$, and $Y_{n}(z)=d_{n}^{-1}\left(\hat{m}_{n}^{0}\left(t_{0}+\right.\right.$ $\left.\left.d_{n} z\right)-\theta_{0}\right)$, for $z \in\left(-d_{n} t_{0},\left(1-t_{0}\right) d_{n}\right]=:\left(a_{n}, b_{n}\right]$. Further, by definition, we 
have that $X_{n}(z) \equiv Y_{n}(z)$, for all $z \notin\left(a_{n}, b_{n}\right]$ and therefore the integrals in (23) (main paper) are finite.

By the charecterization $\hat{m}_{n}(t)$ is constant over $\left(t_{i-1}, t_{i}\right] \equiv((i-1) / n, i / n], i=$ $1, \cdots, n$, and $\hat{m}_{n}^{0}(t)$ is constant over all $\left(t_{i-1}, t_{i}\right] \not \ngtr t_{0}$. Thus,

$$
\begin{aligned}
L_{n} & =\frac{n^{2}}{\sigma_{n}^{2}}\left(\int_{0}^{1}\left(\hat{m}_{n}(t)-\theta_{0}\right)^{2} d t-\int_{0}^{1}\left(\hat{m}_{n}^{0}(t)-\theta_{0}\right)^{2} d t\right)+R_{n} \\
& =\frac{n^{2} d_{n}^{3}}{\sigma_{n}^{2}} \int_{\left(a_{n}, b_{n}\right]}\left(X_{n}^{2}(z)-Y_{n}^{2}(z)\right) d z+R_{n},
\end{aligned}
$$

where $R_{n}$ is given below and where the last relation follows by the change of variables to local coordinates $z=d_{n}^{-1}\left(t-t_{0}\right)$.

Since the only interval $\left(t_{i-1}, t_{i}\right], i=1, \cdots, n$ where $\hat{m}_{n}^{0}(t)$ is potentially non constant is the one containing $t_{0}$, i.e. $i=\left[n t_{0}\right]+1=l+1$, we get

$$
R_{n}=\frac{n^{2}}{\sigma_{n}^{2}}\left(\int_{t_{l}}^{t_{l+1}}\left(\hat{m}_{n}^{0}(t)-\theta_{0}\right)^{2} d t-\frac{1}{n}\left(\hat{m}_{n}^{0}\left(t_{l+1}\right)-\theta_{0}\right)^{2}\right) .
$$

By the monotonicity of $\hat{m}_{n}^{0}(t)$, we have $\left(\hat{m}_{n}^{0}\left(t_{l+1}\right)-\theta_{0}\right)^{2} \leq\left(\hat{m}_{n}^{0}(t)-\theta_{0}\right)^{2}+$ $\left(\hat{m}_{n}^{0}(s)-\theta_{0}\right)^{2}$, for all $t \leq t_{l+1} \leq s$, which implies

$$
R_{n} \leq \frac{2 n^{2}}{\sigma_{n}^{2}} \int_{t_{l}}^{t_{l+2}}\left(\hat{m}_{n}^{0}(t)-\theta_{0}\right)^{2} d t=\frac{2 n^{2} d_{n}^{3}}{\sigma_{n}^{2}} \int_{\Delta_{n}} Y_{n}^{2}(z) d z
$$

where $\Delta_{n}:=d_{n}^{-1}\left(\left[n t_{0}\right] / n-t_{0},\left[n t_{0}\right] / n+2 / n-t_{0}\right) \subset\left[-1 / n d_{n}, 3 / n d_{n}\right]$.

By Theorem A.2, we have that $Y_{n} \Rightarrow \mathcal{S}_{a, b}^{0}$, and since $\Delta_{n}$ is a shrinking interval around 0 the Portmanteau Theorem implies that for all $\epsilon>0$ and $\delta>0$

$$
\begin{aligned}
\limsup _{n \rightarrow \infty} \mathbb{P}\left(\int_{\Delta_{n}} Y_{n}^{2}(z) d z \geq \epsilon\right) & \leq \limsup _{n \rightarrow \infty} \mathbb{P}\left(\int_{-\delta}^{\delta} Y_{n}^{2}(z) d z \geq \epsilon\right) \\
& \leq \mathbb{P}\left(\int_{-\delta}^{\delta}\left(\mathcal{S}_{a, b}^{0}(z)\right)^{2} d z \geq \epsilon\right)
\end{aligned}
$$

As shown in the proof of Theorem 3.2 of Supplement B, $\mathcal{S}_{a, b}^{0}(z)$ is zero in a neighborhood of 0 , and therefore $\int_{-\delta}^{\delta}\left(\mathcal{S}_{a, b}^{0}(z)\right)^{2} d z \rightarrow 0$, as $\delta \downarrow 0$, in probability. Therefore the right-hand side of (16) can be made arbitrarily small. This implies $\int_{\Delta_{n}} Y_{n}^{2}(z) d z \rightarrow 0$, in probability, as $n \rightarrow \infty$, which which in view of (14) and (15) yields (23) (main paper). The argument for the statistic $T_{n}$ is similar. 
Proof of Lemma A.1: Observe that if for some $M>0, X_{n}(M)=$ $Y_{n}(M)$, then $X_{n}(z)=Y_{n}(z)$, for all $z \geq M$. This is because of Lemma 2.2 from Supplement B and the fact that $X_{n}$ are step functions where the jump points are precisely the points where the GCM of $\mathbb{V}_{n}$ touches the curve. Similarly, $X_{n}(-M)=Y_{n}(-M)$ implies $X_{n}(z)=Y_{n}(z)$, for $z \leq-M$. Therefore, it is enough to show that $\limsup _{n \rightarrow \infty} \mathbb{P}\left(X_{n}(M) \neq Y_{n}(M)\right) \rightarrow 0$, as $M \rightarrow \infty$. The case when $M \rightarrow-\infty$ can be treated similarly.

We claim that if $X_{n}(M) \neq Y_{n}(M)$, then either $\hat{m}_{n}^{0}\left(t_{0}+M d_{n}\right)=\theta_{0}$ or $\hat{m}_{n}\left(t_{0}+M d_{n}\right)=\hat{m}_{n}\left(t_{0}\right)$ (see also page 159, Banerjee (2000)[3]). The proof of this claim will be given at the end of this proof. This, since $\left\{X_{n}(0)=\right.$ $\left.X_{n}(M)\right\}=\left\{\hat{m}_{n}\left(t_{0}\right)=\hat{m}_{n}\left(t_{0}+M d_{n}\right)\right\}$ and $\left\{Y_{n}(M)=0\right\}=\left\{\hat{m}_{n}^{0}\left(t_{0}+M d_{n}\right)=\right.$ $\left.\theta_{0}\right\}$, implies

$$
\left\{X_{n}(M) \neq Y_{n}(M)\right\} \subset\left\{Y_{n}(M)=0\right\} \cup\left\{X_{n}(0)=X_{n}(M)\right\} .
$$

Now as $Y_{n}$ is a non-decreasing step function and $Y_{n}(0)=0$,

$$
\begin{aligned}
\limsup _{n \rightarrow \infty} \mathbb{P}\left(Y_{n}(M)=0\right) & \leq \limsup _{n \rightarrow \infty} \mathbb{P}\left(\int_{0}^{M} Y_{n}^{2}(z) d z=0\right) \\
& \leq \mathbb{P}\left(\int_{0}^{M}\left(\mathcal{S}_{a, b}^{0}(z)\right)^{2} d z=0\right),
\end{aligned}
$$

where the last inequality follows from (29) of main paper and the Portmanteau Theorem (see e.g. page 16 of Billingsley, 1999[5]). Similarly, since $X_{n}$ is non-decreasing

$$
\begin{aligned}
\limsup _{n \rightarrow \infty} \mathbb{P}\left(X_{n}(0)=X_{n}(M)\right) & \leq \limsup _{n \rightarrow \infty} \mathbb{P}\left(\int_{0}^{1} X_{n}^{2}(z) d z-\int_{M-1}^{M} X_{n}^{2}(z) d z=0\right) \\
& \leq \mathbb{P}\left(\int_{0}^{1} \mathcal{S}_{a, b}(z)^{2}(z) d z-\int_{M-1}^{M} \mathcal{S}_{a, b}(z)^{2} d z=0\right)
\end{aligned}
$$

by (29) (main paper) and the Portmanteau Theorem. Now, observe that by Lemma 3.2 from Supplement B, we have that the right-hand sides of (17) and (18) vanish, as $M \rightarrow \infty$. This implies the desired inequality.

Now to prove the claim that $X_{n}(M) \neq Y_{n}(M)$ implies either $\hat{m}_{n}^{0}\left(t_{0}+\right.$ $\left.M d_{n}\right)=\theta_{0}$ or $\hat{m}_{n}\left(t_{0}+M d_{n}\right)=\hat{m}_{n}\left(t_{0}\right)$ recall (22) of the main paper. Suppose that $\hat{m}_{n}^{0}\left(t_{0}+M d_{n}\right) \neq \theta_{0}$ and $\hat{m}_{n}\left(t_{0}+M d_{n}\right) \neq \hat{m}_{n}\left(t_{0}\right)$. Note that $\hat{m}_{n}(t)=$ $\mathcal{L} \circ T_{(0,1]}\left(U_{n}\right)(t)$ is a step-function which changes only at points $t$, where the GCM $T_{(0,1]}\left(U_{n}\right)(t)$ of $U_{n}$ equals the function value $U_{n}(t), t \in(0,1]$. Therefore, the fact that $\hat{m}_{n}\left(t_{0}\right) \neq \hat{m}_{n}\left(t_{0}+M d_{n}\right)$, implies that for some 
$t^{*} \in\left(t_{0}, t_{0}+M d_{n}\right]$, we have $T_{(0,1]}\left(U_{n}\right)\left(t^{*}\right)=U_{n}\left(t^{*}\right)$. Note, however, that the constrained GCM $T_{\left(t_{l}, 1\right]}\left(U_{n}\right)(t), t \in\left(t_{l}, 1\right]$ lies between the unconstrained one and the function, i.e.

$$
T_{(0,1]}\left(U_{n}\right)(t) \leq T_{\left(t_{l}, 1\right]}\left(U_{n}\right)(t) \leq U_{n}(t), \quad t \in\left(t_{l}, 1\right] .
$$

This implies that $T_{\left(t_{l}, 1\right]}\left(U_{n}\right)\left(t^{*}\right)=T_{(0,1]}\left(U_{n}\right)\left(t^{*}\right)=U_{n}\left(t^{*}\right)$ and as in the proof of Lemma 2.2 (2) from Supplement B, the two GCMs coincide over the interval $\left[t^{*}, 1\right]$, and so do their slopes

$$
\mathcal{L} \circ T_{\left(t_{l}, 1\right]}\left(U_{n}\right)(t) \equiv \mathcal{L} \circ T_{(0,1]}\left(U_{n}\right)(t), t \in\left(t^{*}, 1\right] .
$$

On there other hand, since $\hat{m}_{n}^{0}\left(t_{0}+M d_{n}\right)=\max \left\{\theta_{0}, \mathcal{L} \circ T_{\left(t_{l}, 1\right]}\left(U_{n}\right)\left(t_{0}+\right.\right.$ $\left.\left.M d_{n}\right)\right\} \neq \theta_{0}$, we have that $\hat{m}_{n}^{0}\left(t_{0}+M d_{n}\right)=\mathcal{L} \circ T_{\left(t_{l}, 1\right]}\left(U_{n}\right)\left(t_{0}+M d_{n}\right)$, which by (19) implies that $\hat{m}_{n}^{0}\left(t_{0}+M d_{n}\right)=\hat{m}_{n}\left(t_{0}+M d_{n}\right)$, since $t^{*}<t_{0}+M d_{n}$. This completes our proof.

Remark 2.1. Since we do not have convergence of finite dimensional distributions of $\left\{X_{n}(z), Y_{n}(z)\right\}_{z \in \mathbb{R}}$ here we cannot use the techniques used to prove the same version of this Lemma in the iid case (see Page 159 of Banerjee, 2000[3]).

Proof of Theorem A.2: We will show that

$$
\begin{gathered}
\mathbb{G C M}_{n}:=\left(\mathcal{T}\left(\mathbb{V}_{n}\right),\left.\mathcal{T}_{\left(-\infty, l_{n}\right]}\left(\mathbb{V}_{n}\right)\right|^{(-\infty, 0)},\left.\mathcal{T}_{\left(l_{n}, \infty\right)}\left(\mathbb{V}_{n}\right)\right|_{(0, \infty)}\right) \\
\Longrightarrow\left(\mathcal{T}(\mathbb{G}), \mathcal{T}_{(-\infty, 0)}(\mathbb{G}), \mathcal{T}_{(0, \infty)}(\mathbb{G})\right)
\end{gathered}
$$

where $\left.\mathcal{T}_{\left(-\infty, l_{n}\right]}\left(\mathbb{V}_{n}\right)\right|^{(-\infty, 0)}$ denotes the extension of the process $\mathcal{T}_{\left(-\infty, l_{n}\right]}\left(\mathbb{V}_{n}\right)$ to $(-\infty, 0)$. This extension is defined as in (5), i.e., we extend the convex function $\mathcal{T}_{\left(-\infty, l_{n}\right]}\left(\mathbb{V}_{n}\right)$ linearly in $\left[l_{n}, 0\right)$ to maintain convexity. The weak convergence $(20)$ is in the space $\mathcal{C}(\mathbb{R}) \times \mathcal{C}(-\infty, 0) \times \mathcal{C}(0, \infty)$ equipped with the product topology of local uniform convergence on compacta.

If (20) holds, then the result follows from a continuous mapping argument. Indeed, consider the map

$$
J: \mathcal{C}(\mathbb{R}) \times \mathcal{C}(-\infty, 0) \times \mathcal{C}(0, \infty) \rightarrow M(\mathbb{R}) \times M(-\infty, 0) \times M(0, \infty),
$$

defined as $J\left(f, f_{-}, f_{+}\right):=\left(\mathcal{L} f, \mathcal{L} f_{-}, \mathcal{L} f_{+}\right)$, where $M(I)$ denotes the space of monotone real valued functions on an interval $I$ equipped with the topology of $L^{2}$ convergence on compact sets. Observe that with the concatenation map $C_{0}: M(-\infty, 0) \times M(0, \infty) \rightarrow M(\mathbb{R})$ defined in (38) with $h=0$. 
we have

$$
\left(X_{n}, Y_{n}\right)=\left(\left(\mathrm{id}, C_{0}\right) \circ J\right)\left(\mathbb{G}_{\mathbb{C M}}\right),
$$

where id $: M(\mathbb{R}) \rightarrow M(\mathbb{R})$ denotes the identity. By Lemmas 7.2 and 7.3 from Section 7, the maps $J$ and $C_{0}$ are continuous and so is the composition $\left(\left(\mathrm{id}, C_{0}\right) \circ J\right)$. This in view of $(20)$ yields $(29)$ in main paper.

Now to complete the proof, we will use Theorem 2.1 along with the standard converging together Lemma B.1 stated in the main paper as well as the continuity Lemma 2.4 from Supplement B to establish (20). Before proceeding further, first we mention a result that we will use later in the proof. Note that for any interval $I$, not necessarily compact we have $\left\{\mathcal{T}_{I}\left(\mathbb{V}_{n}\right)(z)\right\}_{z \in I}$ converges in distribution to $\left\{\mathcal{T}_{I}(\mathbb{G})(z)\right\}_{z \in I}$ uniformly on compacta. Indeed by Theorem A.1, $\left\{\mathbb{V}_{n}(z)\right\}_{z \in \mathbb{R}}$ converges in distribution to $\{\mathbb{G}(z)\}_{z \in \mathbb{R}}$ as a process uniformly on compact sets. The map $\mathcal{T}_{K}: C(K) \mapsto \mathcal{C}(K)$ is continuous for any compact set $K$, where both the spaces are equipped with topology of uniform convergence. So an application of the Continuous Mapping Theorem gives us the result for any compact interval $I$. If $I$ is not compact we prove the result useing converging together lemma (Lemma B.1 from the main paper) and approximating $I$ by some compact interval. The conditions of the Lemma can be verified using continuous mapping (as argued earlier) and Theorem 2.1. We adopt a similar method to establish joint convergence though it is technically more challenging and involved.

It is enough to show that for any fixed compact intervals $I \subset(-\infty, \infty)$, $I_{-} \subset(-\infty, 0)$ and $I_{+} \subset(0, \infty)$, we have that $(20)$ holds restricted to $\mathcal{C}(I) \times$ $\mathcal{C}\left(I_{-}\right) \times \mathcal{C}\left(I_{+}\right)$, equipped with the uniform topology.

Let us fix such intervals and given $\delta>0$ small and $c>0$ large enough so that $I \subset[-c, c], I_{-} \subset[-c,-1 / c]$ and $I_{+} \subset[-\delta, c]$, define,

$$
\xi_{\delta, c, n}:=\left(\left.\mathcal{T}_{[-c, c]}\left(\mathbb{V}_{n}\right)\right|_{I},\left.\mathcal{T}_{[-c,-1 / c]}\left(\mathbb{V}_{n}\right)\right|_{I_{-}},\left.\mathcal{T}_{[-\delta, c]}\left(\mathbb{V}_{n}\right)\right|_{I_{+}}\right)
$$

Let also

$$
\xi_{\delta, c}:=\left(\left.\mathcal{T}_{[-c, c]}(\mathbb{G})\right|_{I},\left.\mathcal{T}_{[-c,-1 / c]}(\mathbb{G})\right|_{I_{-}},\left.\mathcal{T}_{[-\delta, c]}(\mathbb{G})\right|_{I_{+}}\right)
$$

define $\xi:=\left(\left.\mathcal{T}_{(-\infty, \infty)}(\mathbb{G})\right|_{I},\left.\mathcal{T}_{(-\infty, 0)}(\mathbb{G})\right|_{I_{-}},\left.\mathcal{T}_{(0, \infty)}(\mathbb{G})\right|_{I_{+}}\right)$, and finally

$$
\eta_{n}:=\left(\left.\mathcal{T}_{(-\infty, \infty)}\left(\mathbb{V}_{n}\right)\right|_{I},\left.\mathcal{T}_{\left(-\infty, l_{n}\right]}\left(\mathbb{V}_{n}\right) 1_{(-\infty, 0)}\right|_{I_{-}}, \mathcal{T}_{\left(l_{n}, \infty\right)}\left(\mathbb{V}_{n}\right) 1_{(0, \infty)}||_{I_{+}}\right) .
$$

We will verify that $\xi_{\delta, c, n}, \xi_{\delta, c}, \xi$ and $\eta_{n}$ satisfy the conditions of Lemma B.1.

The GCM maps $\mathcal{T}_{[-c, c]}, \mathcal{T}_{[-c,-1 / c]}$ and $\mathcal{T}_{[-\delta, c]}$ are continuous on the spaces $C([-c, c]), C([-c,-1 / c])$ and $C([-\delta, c])$ equipped with the the uniform norm. 
Therefore, by A.1 of the main paper and the Continuous Mapping Theorem, we obtain $\xi_{\delta, c, n} \Rightarrow \xi_{\delta, c}, n \rightarrow \infty$, which verifies condition (i) of Lemma B.1 stated in the main paper.

Since $E=C(I) \times C\left(I_{-}\right) \times C\left(I_{+}\right)$equipped with the uniform topology, it is enough to verify condition (iii) of Lemma B.1 of the main paper for each of the three coordinates separately where $d$ is the uniform metric on the corresponding interval $\left(I, I_{-}\right.$or $\left.I_{+}\right)$. Recall that the processes $V_{n}:=\mathbb{V}_{n}$ satisfy the conditions of Theorem 2.1 and hence (11) implies the condition (iii) for the first coordinate. To verify the condition for the second coordinate we apply Theorem 2.1 with $O_{n}:=\left(-\infty, l_{n}\right], O=(-\infty, 0)$ and $K_{c}=[-c,-1 / c]$. Dealing with the third coordinate is more involved owing to the fact that in Theorem 2.1 the sequence of sets $O_{n}$ increases to $O$, whereas the the intervals $\left[l_{n}, \infty\right) \downarrow(0, \infty)$. So the Theorem does not directly apply. To take care of the third coordinate, we will use Theorem 2.1 along with Lemma 2.4 from Supplement B. Given $\epsilon>0$, we have,

$$
\begin{aligned}
& \lim _{\delta \rightarrow 0} \lim _{c \rightarrow \infty} \limsup _{n \geq 1} \mathbb{P}\left(\sup _{z \in I_{+}}\left|\mathcal{T}_{[-\delta, c]}\left(\mathbb{V}_{n}\right)(z)-\mathcal{T}_{\left(l_{n}, \infty\right)}\left(\mathbb{V}_{n}\right)(z)\right| \geq \epsilon\right) \\
\leq & \lim _{\delta \rightarrow 0} \lim _{c \rightarrow \infty} \limsup _{n \geq 1} \mathbb{P}\left(\sup _{z \in I_{+}}\left|\mathcal{T}_{[-\delta, c]}\left(\mathbb{V}_{n}\right)(z)-\mathcal{T}_{[-\delta, \infty)}\left(\mathbb{V}_{n}\right)(z)\right| \geq \epsilon / 2\right) \\
& +\lim _{\delta \rightarrow 0} \lim _{c \rightarrow \infty} \limsup _{n \geq 1} \mathbb{P}\left(\sup _{z \in I_{+}}\left|\mathcal{T}_{[-\delta, \infty)}\left(\mathbb{V}_{n}\right)(z)-\mathcal{T}_{\left(l_{n}, \infty\right)}\left(\mathbb{V}_{n}\right)(z)\right| \geq \epsilon / 2\right)
\end{aligned}
$$

The first term in the right hand side is 0 by Theorem 2.1. Note that $l_{n} \uparrow 0$ as $n \rightarrow \infty$, so for given $\delta>0$, for large enough $n$, we have $-\delta<l_{n} \leq$ 0 . Therefore the GCM function $\mathcal{T}_{\left(l_{n}, \infty\right)}\left(\mathbb{V}_{n}\right)(t)$ lies in between the GCMs $\mathcal{T}_{[-\delta, \infty)}\left(\mathbb{V}_{n}\right)(t)$ and $\mathcal{T}_{[0, \infty)}\left(\mathbb{V}_{n}\right)(t)$ for all $t \in I_{+}$. So the second term in (21) is bounded above by

$$
\lim _{\delta \rightarrow 0} \limsup _{n \geq 1} \mathbb{P}\left(\sup _{z \in I_{+}}\left|\mathcal{T}_{[-\delta, \infty)}\left(\mathbb{V}_{n}\right)(z)-\mathcal{T}_{[0, \infty)}\left(\mathbb{V}_{n}\right)(z)\right| \geq \epsilon / 2\right)
$$

One can show that (will be proved at the end)

$$
\sup _{z \in I_{+}}\left|\mathcal{T}_{[0, \infty)}\left(\mathbb{V}_{n}\right)(z)-\mathcal{T}_{[-\delta, \infty)}\left(\mathbb{V}_{n}\right)(z)\right| \leq\left|\mathcal{T}_{[0, \infty)}\left(\mathbb{V}_{n}\right)(0)-\mathcal{T}_{[-\delta, \infty)}\left(\mathbb{V}_{n}\right)(0)\right|
$$


Now using the fact $\mathcal{T}_{[0, \infty)}\left(\mathbb{V}_{n}\right)(0)=0$ and $(22)$, the second term in (21) can be bounded above by:

$$
\lim _{\delta \rightarrow 0} \limsup _{n \geq 1} \mathbb{P}\left(\left|\mathcal{T}_{[-\delta, \infty)}\left(\mathbb{V}_{n}\right)(0)\right| \geq \epsilon / 2\right) \leq \lim _{\delta \rightarrow 0} \mathbb{P}\left(\left|\mathcal{T}_{[-\delta, \infty)}(\mathbb{G})(0)\right| \geq \epsilon / 2\right),
$$

where the last inequality follows from the Portmanteau Theorem and the fact that $\mathcal{T}_{[-\delta, \infty)}\left(\mathbb{V}_{n}\right)$ converges in distribution to $\mathcal{T}_{[-\delta, \infty)}(\mathbb{G})$ uniformly on compact set as mentioned earlier. The last quantity in (23) is zero by Lemma 2.4 from Supplement B (since the sample paths of $\mathbb{G}$ satisfy the conditions of that lemma with probability 1 ), which completes the proof of condition (iii) of Lemma B.1 of the main paper.

It was shown in Theorem 1 of $\mathrm{AH}[2]$ that Theorem 2.1 applies to the processes $V_{n}:=\mathbb{G}$. Thus using similar arguments as above applying Relation (11) and Lemma 2.4f of Supplement B we can show that $\xi_{\delta, c} \Rightarrow \xi$, as $c \rightarrow \infty$ and $\delta \uparrow 0$ (in fact the convergence is in probability).

Now it remains to prove (22) to complete the proof. To prove this first notice that, $\mathcal{T}_{[-\delta, \infty)}\left(\mathbb{V}_{n}\right)(z) \leq \mathcal{T}_{[0, \infty)}\left(\mathbb{V}_{n}\right)(z)$ for $z \in[0, \infty)$ and if for some $z_{*} \geq 0$ we have $\mathcal{T}_{[-\delta, \infty)}\left(\mathbb{V}_{n}\right)\left(z_{*}\right)=\mathcal{T}_{[0, \infty)}\left(\mathbb{V}_{n}\right)\left(z_{*}\right)$ then the two GCMs coincide on $\left[z_{*}, \infty\right)$. Let, $z_{*}=\inf \left(z \geq 0: \mathcal{T}_{[-\delta, \infty)}\left(\mathbb{V}_{n}\right)(z)=\mathcal{T}_{[0, \infty)}\left(\mathbb{V}_{n}\right)(z)\right)$. If $z_{*}=0,(22)$ is trivial, otherwise as argued in Lemma A.1 of AH[2], the GCM $\mathcal{T}_{[-\delta, \infty)}\left(\mathbb{V}_{n}\right)(z)$ is a linear function for $z \in\left[0, z_{*}\right]$. Therefore the left slope $\mathcal{L} \circ \mathcal{T}_{[-\delta, \infty)}\left(\mathbb{V}_{n}\right)(z) \equiv \mathcal{L} \circ \mathcal{T}_{[-\delta, \infty)}\left(\mathbb{V}_{n}\right)\left(z_{*}\right) \equiv$ const., for all $z \in\left[0, z_{*}\right)$. Moreover by the fact that $\mathcal{T}_{[-\delta, \infty)}\left(\mathbb{V}_{n}\right)\left(z_{*}\right)=\mathcal{T}_{[0, \infty)}\left(\mathbb{V}_{n}\right)\left(z_{*}\right)$, and domination we get $\mathcal{L} \circ \mathcal{T}_{[0, \infty)}\left(\mathbb{V}_{n}\right)\left(z_{*}\right) \leq \mathcal{L} \circ \mathcal{T}_{[-\delta, \infty)}\left(\mathbb{V}_{n}\right)\left(z_{*}\right)$. This since $z \mapsto \mathcal{T}_{[0, \infty)}\left(\mathbb{V}_{n}\right)(z)$ is a non-decreasing function while $z \mapsto \mathcal{T}_{[-\delta, \infty)}\left(\mathbb{V}_{n}\right)\left(z_{*}\right)$ is constant on $\left(0, z_{*}\right)$, implies that the slope $\mathcal{L} \circ\left(\mathcal{T}_{[0, \infty)}\left(\mathbb{V}_{n}\right)(z)-\mathcal{T}_{[-\delta, \infty)}\left(\mathbb{V}_{n}\right)(z)\right) \leq 0$ for all $z \in\left(0, z_{*}\right)$. This shows that the function $z \mapsto \mathcal{T}_{[0, \infty)}\left(\mathbb{V}_{n}\right)(z)-\mathcal{T}_{[-\delta, \infty)}\left(\mathbb{V}_{n}\right)(z)$ is monotone non-increasing on $\left[0, z_{*}\right]$ and $(22)$ holds. This completes the proof.

\section{Behavior of the Statistics $L_{n}(\theta), T_{n}(\theta)$ and $R_{n}(\theta)$}

The following Lemmas describe the shape of the statistics we have discussed in the main paper.

Lemma 3.1. Define $L_{n}$ and $T_{n}$ as in (4) from main paper. We have $L_{n} \geq$ $T_{n}$. 
Proof. Note that $L_{n} \geq T_{n}$ is equivalent to

$$
\begin{aligned}
& \sum_{i=1}^{n}\left(Y_{i}-\hat{m}_{n}^{0}\left(t_{i}\right)\right)^{2}-\sum_{i=1}^{n}\left(Y_{i}-\hat{m}_{n}\left(t_{i}\right)\right)^{2} \geq \sum_{i=1}^{n}\left(\hat{m}_{n}\left(t_{i}\right)-\hat{m}_{n}^{0}\left(t_{i}\right)\right)^{2} \\
\Longleftrightarrow & \sum_{i=1}^{n}\left(Y_{i}-\hat{m}_{n}\left(t_{i}\right)\right)^{2}+\sum_{i=1}^{n}\left(Y_{i}-\hat{m}_{n}^{0}\left(t_{i}\right)\right)\left(Y_{i}-\hat{m}_{n}\left(t_{i}\right)\right) \leq 0 \\
\Longleftrightarrow & \sum_{i=1}^{n}\left(Y_{i}-\hat{m}_{n}\left(t_{i}\right)\right)\left(\hat{m}_{n}^{0}\left(t_{i}\right)-\hat{m}_{n}\left(t_{i}\right)\right) \leq 0 .
\end{aligned}
$$

Notice that by the definition of isotonic regression (recall (2) from main paper) the vector $\overrightarrow{\hat{m}}:=\left(\hat{m}_{n}\left(t_{i}\right)\right)_{i=1}^{n}$ is the projection of the vector $\vec{y}=$ $\left(y_{i}\right)_{i=1}^{n}$ onto the convex set $V:=\left\{\vec{x}=\left(x_{i}\right)_{i=1}^{n}: x_{1} \leq x_{2} \leq \cdots \leq x_{n}\right\}$. The vector ${\overrightarrow{m^{0}}}^{0}:=\left(\hat{m}_{n}^{0}\left(t_{i}\right)\right)_{i=1}^{n}$ is in $V$. So by the well-known characterization of projections onto closed convex sets we have $(\vec{y}-\overrightarrow{\hat{m}})^{T}\left(\overrightarrow{\hat{m}^{0}}-\overrightarrow{\hat{m}}\right) \leq 0$. The last inequality is equivalent to (24). Hence we have the result.

Lemma 3.2. Both $L_{n}(\theta)$ and $T_{n}(\theta)$ are continuous in $\theta, L_{n}\left(\hat{\theta}_{n}\right)=T_{n}\left(\hat{\theta}_{n}\right)=$ 0 and monotone non-increasing on $\left(-\infty, \hat{\theta}_{n}\right]$ and monotone non-decreasing on $\left(\hat{\theta}_{n}, \infty\right)$. Also $L_{n}(\theta)$ and $T_{n}(\theta)$ diverge to $\infty$ as $\theta$ goes to $\infty$ or $-\infty$.

Proof. Let $\tilde{m}_{n}(t)$ be the left derivative of greatest convex minorant of $U_{n}(t)$ fitted separately for left and right side of $t_{l}$. Then the (constrained) estimate of $m$ under the constraint $m\left(t_{0}\right)=\theta$ is given by $(27)$ in the main paper. Also, if $\hat{\theta}_{n}$ is the isotonic regression estimate of $m\left(t_{0}\right)$, then $\hat{m}_{n}(t)=\hat{m}_{n}^{\hat{\theta}_{n}}(t)$.

Let, $a_{1}<a_{2}<\cdots<a_{m}$ be the distinct values of $\tilde{m}_{n}(t)$ and the corresponding design points are $s_{1}<s_{2}<\cdots<s_{m}$. Also, let, $s_{k}<t_{0}<s_{k+1}$. Then $L_{n}(\theta)$ can be written as

$$
\begin{aligned}
L_{n}(\theta)= & \frac{n}{\sigma_{n}^{2}} \sum_{i=1}^{n}\left[\left(\left(\hat{m}_{n}\left(t_{i}\right)-\theta\right)^{2}-\left(\hat{m}_{n}^{\theta}\left(t_{i}\right)-\theta\right)^{2}\right)\right] \\
= & \frac{n}{\sigma_{n}^{2}} \sum_{i=1}^{k}\left(\left(a_{i} \wedge \hat{\theta}_{n}-\theta\right)^{2}-\left(a_{i} \wedge \theta-\theta\right)^{2}\right) \\
& +\frac{n}{\sigma_{n}^{2}} \sum_{i=k+1}^{m}\left(\left(a_{i} \vee \hat{\theta}_{n}-\theta\right)^{2}-\left(a_{i} \vee \theta-\theta\right)^{2}\right)
\end{aligned}
$$


From (26) of the main paper it follows that $L_{n}\left(\hat{\theta}_{n}\right)=0, L_{n}$ is continuous in $\theta$ and it diverges to $\infty$ as $|\theta| \rightarrow \infty$. The fact that $L_{n}(\theta)$ is monotone nonincreasing on $\left(\infty, \hat{\theta}_{n}\right]$ and monotone non-decreasing on $\left(\hat{\theta}_{n}, \infty\right)$ is argued considering $\theta$ in different intervals and using simple algebra.

We also have

$$
T_{n}(\theta)=\frac{n}{\sigma_{n}^{2}}\left[\sum_{i=1}^{k}\left(a_{i} \wedge \hat{\theta}_{n}-a_{i} \wedge \theta\right)^{2}+\sum_{i=k+1}^{m}\left(a_{i} \vee \hat{\theta}_{n}-a_{i} \vee \theta\right)^{2}\right],
$$

and similar arguments will show the results for $T_{n}(\theta)$.

Proposition 3.1. At the jump points of isotonic regression estimator $L_{n}(\theta)=$ $T_{n}(\theta)$ for all values of $\theta$.

Proof. Note that with the formulation as in the proof of Lemma 3.2, at the jump points we have $a_{i} \vee \hat{\theta}_{n}=a_{i}$ and $a_{i} \wedge \hat{\theta}_{n}=a_{i}$ for all $i$. Now without loss of generality assume that $a_{l-1}<\theta \leq a_{l}$ and $l \leq k$. The other cases can be handled similarly. From the representation (25) we can write

$$
L_{n}(\theta)=\frac{n}{\sigma_{n}^{2}} \sum_{i=l+1}^{k}\left(a_{i}-\theta\right)^{2} .
$$

Similar calculations yield the same form for $T_{n}(\theta)$. Hence the result.

To prove the next result, we need one preliminary result first.

Lemma 3.3. Assume that a sequence of stochastic process $\left\{m_{n}(t)\right\}_{t \in[a, b]}$ converges to $\{m(t)\}_{t \in[a, b]}$ in finite dimensional distributions. The functions $m_{n}$ are monotone non-decreasing and $m$ is continuous monotone non-decreasing and non-random. Then $\left\{m_{n}(t)\right\}_{t \in[a, b]}$ converges to $\{m(t)\}_{t \in[a, b]}$ in distribution uniformly.

Proof. Consider a grid $a=t_{1}<t_{2}<\cdots<t_{k}=b$ such that $\| m\left(t_{i}\right)-$ $m\left(t_{(i+1)}\right) \|<\epsilon$ for some given $\epsilon>0$. Then by monotonicity of $m_{n}$ and $m$ we have,

$$
\begin{aligned}
\sup _{t \in[a, b]}\left|m_{n}(t)-m(t)\right|= & \max _{i=1,2, \ldots n}\left(\left|m_{n}\left(t_{i}\right)-m\left(t_{(i+1)}\right)\right| \vee\left|m_{n}\left(t_{(i+1)}\right)-m\left(t_{i}\right)\right|\right) \\
\leq & \max _{i=1,2, \ldots k}\left(\left|m_{n}\left(t_{i}\right)-m\left(t_{i}\right)\right|+\left|m\left(t_{i}\right)-m\left(t_{(i+1)}\right)\right|\right. \\
& \left.+\left|m_{n}\left(t_{(i+1)}\right)-m\left(t_{(i+1)}\right)\right|+\left|m\left(t_{i+1}\right)-m\left(t_{i}\right)\right|\right) \\
< & 2 \epsilon+\max _{i=1,2, \ldots k}\left(\left|m_{n}\left(t_{i}\right)-m\left(t_{i}\right)\right|+\left|m_{n}\left(t_{(i+1)}\right)-m\left(t_{(i+1)}\right)\right|\right)
\end{aligned}
$$


The second term converges to zero in probability because of the finite dimensional convergence of $m_{n}$ to $m$. As $\epsilon>0$ is arbitrary this implies that $\left\{m_{n}(t)\right\}_{t \in[a, b]}$ converges to $\{m(t)\}_{t \in[a, b]}$ in probability uniformly and hence in distribution.

Proposition 3.2. Let $\theta \neq \theta_{0}$ and $R_{n}(\theta)$ be the ratio statistic calculated under the restriction $m\left(t_{0}\right)=\theta$. Then, under $H_{0}: m\left(t_{0}\right)=\theta_{0}, R_{n}(\theta) \stackrel{P}{\rightarrow} 1$ as $n \rightarrow \infty$.

Proof. Assume $\theta>\theta_{0}$. By AH[2], $\hat{m}_{n}(t) \stackrel{P}{\rightarrow} m(t)$ for all $t \in(0,1)$. Let $\tilde{m}_{n}(t)$ be the slope of the GCM of $U_{n}(t)$ ((25) from main paper) where the GCM is fitted separately at left and right of $t_{l}$, the nearest design point at the left of $t_{0}$. The isotonic regression estimate of $m(t)$ under the constraint $m\left(t_{0}\right)=\theta$ is given by

$$
\hat{m}_{n}^{\theta}(t)= \begin{cases}\tilde{m}_{n}(t) \wedge \theta, & \text { if } t \leq t_{l} \\ \theta, & \text { if } t_{l}<t \leq t_{0} \\ \tilde{m}_{n}(t) \vee \theta, & \text { if } t>t_{0} .\end{cases}
$$

Considering the regression problem on the intervals $\left[0, t_{l}\right]$ and $\left[t_{l}, 1\right]$ separately and by the fact $t_{l} \rightarrow t_{0}$ as $n \rightarrow \infty$, we have for $t \in\left(0, t_{0}\right)$, $\hat{m}_{n}^{\theta}(t) \stackrel{P}{\rightarrow} m(t) \wedge \theta=m(t)$ and for $t \in\left(t_{0}, 1\right), \hat{m}_{n}^{\theta}(t) \stackrel{P}{\rightarrow} m(t) \vee \theta$. So, $\hat{m}_{n}^{\theta}(t) \stackrel{P}{\rightarrow} m(t) \mathbf{1}_{\left(t<t_{0}\right)}+(m(t) \vee \theta) \mathbf{1}_{\left(t \geq t_{0}\right)}:=m^{\theta}(t)$ for all $t \in(0,1)$.

Now as $\hat{m}_{n}(t) \stackrel{P}{\rightarrow} m(t)$, for any $0<a<b<1\left\{\hat{m}_{n}(t)\right\}_{t \in[a, b]} \rightarrow$ $\{m(t)\}_{t \in[a, b]}$ in finite dimensional distribution. Also, $\hat{m}_{n}(t)$ is increasing and $m(t)$ is continuous, increasing and non random. So Lemma 3.3 implies that $\left\{\hat{m}_{n}(t)\right\}_{t \in[a, b]} \Rightarrow\{m(t)\}_{t \in[a, b]}$ uniformly on $D[a, b]$. As $m(t)$ is non-random this implies that $\left\{\hat{m}_{n}(t)\right\}_{t \in[a, b]}$ converges in probability to $\{m(t)\}_{t \in[a, b]}$ in $D[a, b]$. Similar arguments can be applied to establish the convergence of $\left\{\hat{m}_{n}^{\theta}(t)\right\}_{t \in[a, b]}$ to $\left\{m^{\theta}(t)\right\}_{t \in[a, b]}$ in probability as a process in $D[a, b]$. So, $\left\{\hat{m}_{n}(t), \hat{m}_{n}^{\theta}(t)\right\}_{t \in[a, b]}$ converges jointly to $\left\{m(t), m^{\theta}(t)\right\}_{t \in[a, b]}$ in probability. 
Now look at the statistic $R_{n}(\theta)$ :

$$
\begin{aligned}
R_{n}(\theta) & =\frac{L_{n}(\theta)}{T_{n}(\theta)}=\frac{\sum_{i=1}^{n}\left(\left(\hat{m}_{n}\left(t_{i}\right)-\theta\right)^{2}-\left(\hat{m}_{n}^{\theta}\left(t_{i}\right)-\theta\right)^{2}\right)}{\sum_{i=1}^{n}\left(\left(\hat{m}_{n}\left(t_{i}\right)-\theta\right)-\left(\hat{m}_{n}^{\theta}\left(t_{i}\right)-\theta\right)\right)^{2}} \\
& =\frac{\int_{0}^{1}\left(\left(\hat{m}_{n}(t)-\theta\right)^{2}-\left(\hat{m}_{n}^{\theta}(t)-\theta\right)^{2}\right) d t}{\int_{0}^{1}\left(\left(\hat{m}_{n}(t)-\theta\right)-\left(\hat{m}_{n}^{\theta}(t)-\theta\right)\right)^{2} d t} \\
= & \frac{1}{1-2 \bar{R}_{n}(\theta)}
\end{aligned}
$$

where,

$$
\bar{R}_{n}(\theta)=\frac{\int_{0}^{1}\left(\hat{m}_{n}^{\theta}(t)-\theta\right)\left(\hat{m}_{n}(t)-\hat{m}_{n}^{\theta}(t)\right) d t}{\int_{0}^{1}\left(\hat{m}_{n}(t)+\hat{m}_{n}^{\theta}(t)-2 \theta\right)\left(\hat{m}_{n}(t)-\hat{m}_{n}^{\theta}(t)\right) d t}
$$

By Lemma A.1 from main paper given $\epsilon>0$ we can find $0<a<b<1$ such that $P\left(\tilde{m}_{n} \neq \hat{m}_{n} \subset[a, b]\right)>1-\epsilon$ for sufficiently large $n$. Pick $a$ and $b$ such that $m(a)<\theta_{0}$ and $m(b)>\theta$. As $\hat{m}_{n}(t)$ converges in probability to $m(t)$ for $t \in(0,1)$ we have $P\left(\hat{m}_{n}(b)>\theta\right)>1-\epsilon$ and $P\left(\hat{m}_{n}(a)<\theta_{0}\right)>1-\epsilon$ for large enough $n$.

Consider the event

$$
A=\left\{\tilde{m}_{n} \neq \hat{m}_{n} \subset[a, b]\right\} \cap\left\{\hat{m}_{n}(b)>\theta\right\} \cap\left\{\hat{m}_{n}(a)<\theta_{0}\right\} .
$$

From above discussion we have $P(A)>1-3 \epsilon$. For all $\omega \in A$, if $t \notin[a, b]$, $\hat{m}_{n}(t) \equiv \tilde{m}_{n}(t)>\theta$, and therefore for $t>b, \hat{m}_{n}^{\theta}(t)=\tilde{m}_{n}(t) \vee \theta=\tilde{m}_{n}(t)=$ $\hat{m}_{n}(t)$ and for $t<a, \hat{m}_{n}^{\theta}(t)=\tilde{m}_{n}(t) \wedge \theta=\tilde{m}_{n}(t)=\hat{m}_{n}(t)$.

So, $\exists[a, b] \subset(0,1)$ such that $P\left(\hat{m}_{n}^{\theta} \neq \hat{m}_{n} \subset[a, b]\right) \rightarrow 1$ as $n \rightarrow \infty$.

So all the integrals in $\bar{R}_{n}(\theta)$ can be considered as integral over $[a, b]$. The integrand of the denominator converges in probability to $\left(m^{\theta}(t)+m(t)-\right.$ $2 \theta)\left(m(t)-m^{\theta}(t)\right)=(m(t)-\theta) \mathbf{1}_{\left(t \geq t_{0}, m(t)<\theta\right)}$. As $m$ is continuous and increasing and $m\left(t_{0}\right)=\theta_{0}<\theta$, so this limiting function is positive on the interval $\left[t_{0}, m^{-1}(\theta) \wedge 1\right]$. And the integrand of the numerator converges in probability to $\left(m^{\theta}(t)-\theta\right)\left(m(t)-m^{\theta}(t)\right)=0$. As the integrals in both numerator 
and denominator are continuous functional of $\hat{m}_{n}$ and $\hat{m}_{n}^{\theta}$ in $L_{[0,1]}^{2}$ we have $\bar{R}_{n}(\theta) \stackrel{P}{\rightarrow} 0$ as $n \rightarrow \infty$. This in turn implies that $R_{n}(\theta) \stackrel{P}{\rightarrow} 1$ as $n \rightarrow \infty$.

Theorem 3.1. Let $L_{n}(\theta, t)$ be the test statistic for testing $H_{0}: m(t)=\theta$. If $t_{1} \neq t_{2}$. If the errors come from a Gaussian distribution, $\left\{L_{n}\left(\theta, t_{1}\right)\right\}_{\theta}$ and $\left\{L_{n}\left(\theta, t_{2}\right)\right\}_{\theta}$ are asymptotically independent.

Proof. Note that following the proof of Theorem A.1 $L_{n}\left(\theta, t_{1}\right)$ is a functional of the partial sum process $\left\{d_{n}^{-2} n^{-1}\left(v_{n}\left(t_{1}+z d_{n}\right)-v_{n}\left(t_{1}\right)\right)\right\}_{z \in \mathbb{R}}$. We shall show the processes $\left\{d_{n}^{-2} n^{-1}\left(v_{n}\left(t_{1}+z d_{n}\right)-v_{n}\left(t_{1}\right)\right)\right\}_{z \in \mathbb{R}}$ and $\left\{d_{n}^{-2} n^{-1}\left(v_{n}\left(t_{2}+z d_{n}\right)-\right.\right.$ $\left.\left.v_{n}\left(t_{2}\right)\right)\right\}_{z \in \mathbb{R}}$ are asymptotically independent which in term will prove the asymptotic independence of $\left\{L_{n}\left(\theta, t_{1}\right)\right\}_{\theta}$ and $\left\{L_{n}\left(\theta, t_{2}\right)\right\}_{\theta}$. By stationarity of the error process for fixed $z$ we can write

$\operatorname{Cov}\left(v_{n}\left(t_{1}+z d_{n}\right)-v_{n}\left(t_{1}\right), v_{n}\left(t_{2}+z d_{n}\right)-v_{n}\left(t_{2}\right)\right)=\operatorname{Cov}\left(\sum_{i=1}^{\left\lfloor n z d_{n}\right\rfloor} \epsilon_{i}, \sum_{i=\left\lfloor n\left(t_{2}-t_{1}\right)\right\rfloor}^{\left\lfloor n\left(t_{2}-t_{1}+z d_{n}\right)\right\rfloor} \epsilon_{i}\right)$.

Therefore, for LRD errors, the covariance turns out to be

$$
\begin{aligned}
& d_{n}^{-4} n^{-2} \operatorname{Cov}\left(\sum_{i=1}^{\left\lfloor n z d_{n}\right\rfloor} \epsilon_{i}, \sum_{i=\left\lfloor n\left(t_{2}-t_{1}\right)\right\rfloor}^{\left\lfloor n\left(t_{2}-t_{1}+z d_{n}\right)\right\rfloor} \epsilon_{i}\right) \\
= & d_{n}^{-4} n^{-2}\left(\operatorname{Cov}\left(\left\lfloor n\left(t_{2}-t_{1}+z d_{n}\right)\right\rfloor\right)+2 \operatorname{Cov}\left(\left\lfloor n\left(t_{2}-t_{1}+z d_{n}\right)\right\rfloor-1\right)+\cdots+\left\lfloor n z d_{n}\right\rfloor \operatorname{Cov}\left(\left\lfloor n\left(t_{2}-t_{1}\right)\right\rfloor\right)\right) \\
= & d_{n}^{-4} n^{-2}\left(\left(\left\lfloor n\left(t_{2}-t_{1}+z d_{n}\right)\right\rfloor\right)^{-d}+2\left(\left\lfloor n\left(t_{2}-t_{1}+z d_{n}\right)\right\rfloor-1\right)^{-d}+\cdots+n z d_{n}\left(\left\lfloor n\left(t_{2}-t_{1}\right)\right\rfloor\right)^{-d}\right) \\
\leq & n^{\frac{4 d}{2+d}} n^{-2} n^{-d}\left(t_{2}-t_{1}+z d_{n}\right)^{-d}\left(1+2+\ldots . n z d_{n}\right) \\
\sim & n^{\frac{4 d}{2+d}} n^{-2} n^{-d}\left(t_{2}-t_{1}+z d_{n}\right)^{-d}\left(n z d_{n}\right)^{2} \\
= & n^{\frac{2 d}{2+d}} n^{-d}\left(t_{2}-t_{1}+z d_{n}\right)^{-d} \\
= & n^{-\frac{d^{2}}{2+d}}\left(t_{2}-t_{1}+z d_{n}\right)^{-d} .
\end{aligned}
$$

The last term goes to 0 as $n \rightarrow \infty$. Similar calculations can be shown for SRD errors. For normal errors this implies independence of $\left\{L_{n}\left(\theta, t_{1}\right)\right\}_{\theta}$ and $\left\{L_{n}\left(\theta, t_{2}\right)\right\}_{\theta}$. The derivation extends readily to asymptotic independence at $k$ different points $t_{1}, t_{2}, \ldots, t_{k}$.

Some additional observations on the $\Psi_{n}(\theta)$ based confidence sets: The quantile $F_{\Psi}^{\leftarrow}(1-\alpha)$ may lie entirely below the graph of the statistic $\Psi_{n}(\theta)$ with some positive probability. In particular, this corresponds to the case 
where $L_{n}(\theta)=T_{n}(\theta)$ for all $\theta$. As shown in Proposition 3.1, this happens at the points where isotonic regression estimator jumps. (Note however for a pre-fixed point of interest the probability of it being a jump point is zero.) In this case, the confidence interval from inversion of the $\Psi$-statistic is the empty set. Also, note that with non-zero probability, the confidence interval based on $\Psi_{n}$ can be the entire range of the function $m$, though this probability, by the observation following Proposition 5.2 (main paper), goes to 0 as $n$ increases.

Also, it is unclear at this point that $\Psi$ is a proper random variable, i.e., $\mathbb{P}(\Psi<$ $\infty)=1$. Extensive simulations suggest that this should be the case, and also that the distribution function is continuous and strictly increasing. It is possible that the distribution of $\mathcal{R}$ may harbor a small mass at the point 1 (and therefore $\Psi$ a mass at $\infty$ ), undetectable by simulations. But Proposition 5.2 (main paper) implies that confidence intervals (at level 100(1- $\alpha) \%$ ) based on $\Psi$ (or equivalently on $\mathcal{R}$ ) would be consistent provided that $\alpha>$ $\mathbb{P}(\mathcal{R}=1)$, since the $(1-\alpha)$ quantile of $\Psi$ would then be finite. Based on our simulations, if such an $\alpha$ does exist it would have be orders of magnitude smaller than .01, so this would have no bearing on the construction of usual confidence intervals.

\section{Local Asymptotic Behavior of $L_{n}$ and $T_{n}$}

In this section we will study the local asymptotic behavior of the statistics $L_{n}(\theta)$ and $T_{n}(\theta)$. This is useful to prove Theorem 4.4 in the main paper, where the rate of the length of the confidence intervals based on $L_{n}$ and $T_{n}$ is obtained.

Let $\theta_{n, h}=\theta_{0}+h d_{n}$ and $\hat{m}_{n}^{h}(t)$ be the solution of the isotonic regression problem under the constraint $m\left(t_{0}\right)=\theta_{n, h}$. For $z \in\left(a_{n}, b_{n}\right]$. Define,

$$
\begin{aligned}
\mathbb{V}_{n}\left(z, \theta_{n, h}\right)=d_{n}^{-2} & \left(U_{n}\left(t_{0}+z d_{n}\right)-U_{n}\left(t_{0}\right)-\theta_{n, h} z d_{n}\right) \\
X_{n}\left(z, \theta_{n, h}\right) & =d_{n}^{-1}\left(\hat{m}_{n}\left(t_{0}+z d_{n}\right)-\theta_{n, h}\right) \\
& =\mathcal{L}\left(\mathcal{T}\left(\mathbb{V}_{n}\left(., \theta_{n, h}\right)\right),\left(a_{n}, b_{n}\right]\right) \\
& =\mathcal{L}\left(\mathcal{T}\left(\left(\mathbb{V}_{n},\left(a_{n}, b\right]\right)\right)-h\right.
\end{aligned}
$$


and

$$
\begin{aligned}
Y_{n}\left(z, \theta_{n, h}\right)= & d_{n}^{-1}\left(\hat{m}_{n}\left(t_{0}+z d_{n}\right)-\theta_{n, h}\right) \\
= & \left(\mathcal{L}\left(\mathcal{T}\left(\mathbb{V}_{n}\left(., \theta_{n, h}\right),\left(a_{n}, l_{n}\right]\right)\right) \wedge 0\right) \mathbf{1}_{\left(a_{n}, l_{n}\right]}+0 \times \mathbf{1}_{\left(l_{n}, 0\right]} \\
& +\left(\mathcal{L}\left(\mathcal{T}\left(\mathbb{V}_{n}\left(., \theta_{n, h}\right),\left(l_{n}, b_{n}\right]\right)\right) \vee 0\right) \mathbf{1}_{\left(0, b_{n}\right]} \\
= & \left(\mathcal{L}\left(\mathcal{T}\left(\mathbb{V}_{n},\left(a_{n}, l_{n}\right]\right)\right) \wedge h\right) \mathbf{1}_{\left(a_{n}, l_{n}\right]}+h \times \mathbf{1}_{\left(l_{n}, 0\right]} \\
& +\left(\mathcal{L}\left(\mathcal{T}\left(\mathbb{V}_{n},\left(l_{n}, b_{n}\right]\right)\right) \vee h\right) \mathbf{1}_{\left(0, b_{n}\right]}-h
\end{aligned}
$$

where $\mathbb{V}_{n}$ is defined as (26) in main paper. Relation (29) follows because $(a-h) \wedge 0=a \wedge h-h$ and $(a-h) \vee 0=a \vee h-h$.

We extend $X_{n}\left(z, \theta_{n, h}\right)$ and $Y_{n}\left(z, \theta_{n, h}\right)$ to be defined for all $z \in \mathbb{R}$ as left-continuous step functions constant outside the interval $\left(a_{n}, b_{n}\right]$.

Theorem 4.1. The process $\left\{\left(X_{n}\left(z, \theta_{n, h}\right), Y_{n}\left(z, \theta_{n, h}\right)\right)\right\}_{z \in \mathbb{R}}$ converges in distribution to $\left\{\left(\mathcal{S}_{a, b}(z)-h, \mathcal{S}_{a, b}^{h}(z)-h\right)\right\}_{z \in \mathbb{R}}$ in $L_{l o c}^{2} \times L_{\text {loc }}^{2}$, where $\mathcal{S}_{a, b}$ and $\mathcal{S}_{a, b}^{h}$ defined in (12) of the main paper.

Proof. By Theorem A.1 from the main paper, we have $\mathbb{V}_{n} \Rightarrow \mathbb{G}$ uniformly on compacta as a process. Therefore as argued in Theorem A.2 therein we have (20):

$$
\begin{gathered}
\mathbb{G C M}_{n}:=\left(\mathcal{T}\left(\mathbb{V}_{n}\right), \mathcal{T}_{\left(-\infty, l_{n}\right]}\left(\mathbb{V}_{n}\right) 1_{(-\infty, 0)}, \mathcal{T}_{\left(l_{n}, \infty\right)}\left(\mathbb{V}_{n}\right) 1_{(0, \infty)}\right) \\
\Longrightarrow\left(\mathcal{T}(\mathbb{G}), \mathcal{T}_{(-\infty, 0)}(\mathbb{G}), \mathcal{T}_{(0, \infty)}(\mathbb{G})\right)
\end{gathered}
$$

in the space $\mathcal{C}(\mathbb{R}) \times \mathcal{C}(-\infty, 0) \times \mathcal{C}(0, \infty)$ equipped with the local uniform convergence on compact sets.

Now as in TheoremA.2 from paper consider

$$
J: \mathcal{C}(\mathbb{R}) \times \mathcal{C}(-\infty, 0) \times \mathcal{C}(0, \infty) \rightarrow M(\mathbb{R}) \times M(-\infty, 0) \times M(0, \infty),
$$

defined as $J\left(f, f_{-}, f_{+}\right):=\left(\mathcal{L} f, \mathcal{L} f_{-}, \mathcal{L} f_{+}\right)$. Define map $\tilde{C}_{h}: M(-\infty, 0) \times$ $M(0, \infty) \rightarrow M(\mathbb{R})$ as $\tilde{C}_{h}\left(f_{1}, f_{2}\right)=C_{h}\left(f_{1}, f_{2}\right)-h$, where $C_{h}$ is the concatenation map in Lemma 7.3. We have

$$
\left(X_{n}, Y_{n}\right)=\left(\left(\mathrm{id}, \tilde{C}_{h}\right) \circ J\right)\left(\mathbb{G} \mathbb{C M}_{n}\right),
$$

where id $: M(\mathbb{R}) \rightarrow M(\mathbb{R})$ denotes the identity. By Lemmas 7.2 and 7.3 from Section 7, the maps $J$ and $C_{h}$ are continuous and so is $\tilde{C}_{h}$ and the composition $\left(\left(\mathrm{id}, \tilde{C}_{h}\right) \circ J\right)$. Hence an application of the Continuous Mapping Theorem gives us the result. 
We will need this result in the sequel.

Corollary 4.1. For every fixed $h \in \mathbb{R}$, we have that $\left(X_{n}(0),\left\{X_{n}\left(z, \theta_{0}+\right.\right.\right.$ $\left.\left.\left.h d_{n}\right), Y_{n}\left(z, \theta_{0}+h d_{n}\right)\right\}_{z \in \mathbb{R}}\right)$ converges in distribution to $\left(\mathcal{S}_{a, b}(0),\left\{\mathcal{S}_{a, b}(z)-\right.\right.$ $\left.\left.h, \mathcal{S}_{a, b}^{h}(z)-h\right\}_{z \in \mathbb{R}}\right)$ in $\mathbb{R} \times L_{l o c}^{2} \times L_{l o c}^{2}$.

Proof. As $f \mapsto \mathcal{T}_{c}(f)$ is a continuous mapping, Theorem A.1 from main paper implies $\mathcal{T}_{K}\left(\mathbb{V}_{n}\right) \Longrightarrow \mathcal{T}_{K}(\mathbb{G})$ as $n \rightarrow \infty$ uniformly on compact sets. So by the arguments in the proof of Theorem 4.1 we have (21) of main paper. Now Lemma 7.2 implies that the map $L: f \mapsto \partial_{\ell} f$ is a continuous map from the space of convex function to $L_{l o c}^{2}$. By AH[2] (page 1890-1891) $\mathcal{S}_{a, b}$ is continuous at 0 almost surely. So Lemma 7.1 from paper and the Continuous Mapping Theorem imply the result.

We shall now obtain expressions for $L_{n}\left(\theta_{n, h}\right)$ and $T_{n}\left(\theta_{n, h}\right)$ through the processes $X_{n}$ and $Y_{n}$. First let $D_{n}^{h}$ be the set on which $\hat{m}_{n}$ and $\hat{m}_{n}^{h}$ differ. Then, for any $\epsilon>0$, we can find $M_{\epsilon}>0$ such that with probability greater than $1-\epsilon, D_{n}^{h} \subset\left[t_{0}-M_{\epsilon} d_{n}, t_{0}+M_{\epsilon} d_{n}\right]$, eventually. The proof is similar to Lemma A.1 (main paper). Let $\tilde{D}_{n}^{h}=d_{n}^{-1}\left(D_{n}^{h}-t_{0}\right)$.

Proposition 4.1. The quantities $L_{n}(\theta)$ and $T_{n}(\theta)$ can be expressed as

$$
\begin{aligned}
& L_{n}\left(\theta_{n, h}\right)=\frac{n^{2} d_{n}^{3}}{\sigma_{n}^{2}}\left(\int_{\mathbb{R}}\left(X_{n}^{2}\left(z, \theta_{n, h}\right)-Y_{n}^{2}\left(z, \theta_{n, h}\right)\right) d z+o_{P}(1)\right) \\
& T_{n}\left(\theta_{n, h}\right)=\frac{n^{2} d_{n}^{3}}{\sigma_{n}^{2}}\left(\int_{\mathbb{R}}\left(X_{n}\left(z, \theta_{n, h}\right)-Y_{n}\left(z, \theta_{n, h}\right)\right)^{2} d z+o_{P}(1)\right)
\end{aligned}
$$

Proof. The proof is basically similar to the proof of Proposition A.1 from 
the main paper.

$$
\begin{aligned}
L_{n}\left(\theta_{n, h}\right)= & \frac{n}{\sigma_{n}^{2}}\left[-\sum_{i=1}^{n}\left(Y_{i}-\hat{m}_{n}\left(t_{i}\right)\right)^{2}+\sum_{i=1}^{n}\left(Y_{i}-\hat{m}_{n}^{h}\left(t_{i}\right)\right)^{2}\right] \\
= & \frac{n}{\sigma_{n}^{2}}\left[2 \sum_{i=1}^{n}\left(Y_{i}-\theta_{n, h}\right)\left(\hat{m}_{n}\left(t_{i}\right)-\theta_{n, h}\right)-2 \sum_{i=1}^{n}\left(Y_{i}-\theta_{n, h}\right)\left(\hat{m}_{n}^{h}\left(t_{i}\right)-\theta_{n, h}\right)\right] \\
& -\frac{n}{\sigma_{n}^{2}}\left[\sum_{i=1}^{n}\left(\hat{m}_{n}\left(t_{i}\right)-\theta_{n, h}\right)^{2}-\sum_{i=1}^{n}\left(\hat{m}_{n}^{h}\left(t_{i}\right)-\theta_{n, h}\right)^{2}\right] \\
= & \frac{n^{2}}{\sigma_{n}^{2}} \sum_{i=1}^{n}\left[\frac{1}{n}\left(\left(\hat{m}_{n}\left(t_{i}\right)-\theta_{n, h}\right)^{2}-\left(\hat{m}_{n}^{h}\left(t_{i}\right)-\theta_{n, h}\right)^{2}\right)\right] \\
= & \frac{n^{2}}{\sigma_{n}^{2}}\left(\int_{0}^{1}\left(\hat{m}_{n}(t)-\theta_{0}\right)^{2} d t-\int_{0}^{1}\left(\hat{m}_{n}^{h}(t)-\theta_{n, h}\right)^{2} d t\right)+R_{n} \\
= & \frac{n^{2} d_{n}^{3}}{\sigma_{n}^{2}} \int_{\mathbb{R}}\left(X_{n}^{2}\left(z, \theta_{n, h}\right)-Y_{n}^{2}\left(z, \theta_{n, h}\right)\right) d z+R_{n}
\end{aligned}
$$

In view of Theorem 4.1 with similar arguments as in the proof of Proposition A.1 we can bound the remainder $R_{n}$ and obtain:

$$
L_{n}\left(\theta_{n, h}\right)=\frac{n^{2} d_{n}^{3}}{\sigma_{n}^{2}}\left(\int_{\mathbb{R}}\left(X_{n}^{2}\left(z, \theta_{n, h}\right)-Y_{n}^{2}\left(z, \theta_{n, h}\right)\right) d z+o_{P}(1)\right) .
$$

Similarly we have,

$$
T_{n}\left(\theta_{n, h}\right)=\frac{n^{2} d_{n}^{3}}{\sigma_{n}^{2}}\left(\int_{\mathbb{R}}\left(X_{n}\left(z, \theta_{n, h}\right)-Y_{n}\left(z, \theta_{n, h}\right)\right)^{2} d z+o_{P}(1)\right) .
$$

Let $D_{a, b}^{h}$ be the set where $\mathcal{S}_{a, b}(z)$ and $\mathcal{S}_{a, b}^{h}(z)$ differ. Define,

$$
\begin{aligned}
L_{\infty}(h)= & \frac{1}{a^{2}} \int_{D_{a, b}^{h}}\left(\left(\mathcal{S}_{a, b}(z)\right)^{2}-\left(\mathcal{S}_{a, b}^{h}(z)\right)^{2}\right) d z \\
& -\frac{2 h}{a^{2}} \int_{D_{a, b}^{h}}\left(\mathcal{S}_{a, b}(z)-\mathcal{S}_{a, b}^{h}(z)\right) d z, \\
T_{\infty}(h)= & \frac{1}{a^{2}} \int_{D_{a, b}^{h}}\left(\mathcal{S}_{a, b}(z)-\mathcal{S}_{a, b}^{h}(z)\right)^{2} d z .
\end{aligned}
$$

For long range dependent errors $L_{\infty}(h)$ and $T_{\infty}(h)$ depend on $r$ and $H$. 
Remark 4.1. Note that $L_{\infty}(0)=\frac{1}{a^{2}} \mathbb{L}_{a, b}$ and $T_{\infty}(0)=\frac{1}{a^{2}} \mathbb{T}_{a, b}$ where $\mathbb{L}_{a, b}$ and $\mathbb{T}_{a, b}$ in (13) of the main paper.

Theorem 4.2. As $n \rightarrow \infty$ the process $\left\{f(n)\left(L_{n}\left(\theta_{0}+h d_{n}\right), T_{n}\left(\theta_{0}+h d_{n}\right)\right)\right\}_{h \in \mathbb{R}}$ converges to $\left\{\left(L_{\infty}(h), T_{\infty}(h)\right)\right\}_{h \in \mathbb{R}}$ in finite dimensional distributions, where

$$
f(n)= \begin{cases}1 & \text { under weak dependence } \\ n^{-\frac{(1-H)(1-2 H)}{(2-H)}} & \text { under strong dependence. }\end{cases}
$$

Proof. Consider $\theta_{n, h_{1}}, \theta_{n, h_{2}}, \ldots, \theta_{n, h_{k}}$ for $1 \leq i \leq k$. The arguments presented in the proof of Theorem 4.1 can be easily extended to show that $\left(X_{n}\left(., \theta_{n, h_{i}}\right), Y_{n}\left(., \theta_{n, h_{i}}\right): 1 \leq i \leq k\right)$ converges in distribution to $\left(S_{a, b}()-\right.$. $\left.h_{i}, S_{a, b}^{h_{i}(.)}-h_{i}: 1 \leq i \leq k\right)$ under $\left(L_{l o c}^{2}\right)^{2 k}$ metric.

Note that for the term $n^{2} d_{n}^{3} / \sigma_{n}^{2}$ converges to $1 / a^{2}$ for SRD and for LRD it turns out to be $n^{\frac{(1-H)(1-2 H)}{(2-H)}}\left(1 / a^{2}\right)$.

The rest of the proof involves a localization argument similar to the one in the proof of Theorem 4.2 (main paper). Given $\epsilon>0$, we can get a compact set $K_{\epsilon}$ of the form $\left[-M_{\epsilon}, M_{\epsilon}\right]$ such that for $1 \leq i \leq k$,

$$
\mathbb{P}\left[\tilde{D}_{n}^{h_{i}} \subset\left[-M_{\epsilon}, M_{\epsilon}\right]\right]>1-\frac{\epsilon}{2 k} \text { and } \mathbb{P}\left[D_{a, b}^{h_{i}} \subset\left[-M_{\epsilon}, M_{\epsilon}\right]\right]>1-\frac{\epsilon}{2 k} .
$$

Define,

$$
\begin{aligned}
& V_{n, \epsilon, i}=\frac{1}{a^{2}} \int_{K_{\epsilon}}\left(X_{n}^{2}\left(z, \theta_{n, h_{i}}\right)-Y_{n}^{2}\left(z, \theta_{n, h_{i}}\right)\right) d z \\
& W_{\epsilon, i}=\frac{1}{a^{2}} \int_{K_{\epsilon}}\left(\left(\mathcal{S}_{a, b}(z)\right)^{2}-\left(\mathcal{S}_{a, b}^{h_{i}}(z)\right)^{2}\right) d z-\frac{2 h_{i}}{a^{2}} \int_{K_{\epsilon}}\left(\left(\mathcal{S}_{a, b}(z)\right)-\left(\mathcal{S}_{a, b}^{h_{i}}(z)\right)\right) d z \\
& V_{n, \epsilon,(i+k)}=\frac{1}{a^{2}} \int_{K_{\epsilon}}\left(X_{n}\left(z, \theta_{n, h_{i}}\right)-Y_{n}\left(z, \theta_{n, h_{i}}\right)\right)^{2} d z \\
& W_{\epsilon,(i+k)}=\frac{1}{a^{2}} \int_{K_{\epsilon}}\left(\mathcal{S}_{a, b}(z)-\mathcal{S}_{a, b}^{h_{i}}(z)\right)^{2} d z .
\end{aligned}
$$

Also, introduce the quantities

$$
\begin{aligned}
& \xi_{n i}=\frac{1}{a^{2}} \int_{\tilde{D}_{n}^{h_{i}}}\left(X_{n}^{2}\left(z, \theta_{n, h_{i}}\right)-Y_{n}^{2}\left(z, \theta_{n, h_{i}}\right)\right) d z, \\
& \xi_{i}=\frac{1}{a^{2}} \int_{D_{a, b}^{h_{i}}}\left(\left(\mathcal{S}_{a, b}(z)\right)^{2}-\left(\mathcal{S}_{a, b}^{h_{i}}(z)\right)^{2}\right) d z-\frac{2 h_{i}}{a^{2}} \int_{D_{a, b}^{i}}\left(\left(\mathcal{S}_{a, b}(z)\right)-\left(\mathcal{S}_{a, b}^{h_{i}}(z)\right)\right) d z \\
& \xi_{n(i+k)}=\frac{1}{a^{2}} \int_{\tilde{D}_{n}^{h_{i}}}\left(X_{n}\left(z, \theta_{n, h_{i}}\right)-Y_{n}\left(z, \theta_{n, h_{i}}\right)\right)^{2} d z \\
& \xi_{i+k}=\frac{1}{a^{2}} \int_{D_{a, b}^{h_{i}}}\left(\mathcal{S}_{a, b}(z)-\mathcal{S}_{a, b}^{h_{i}}(z)\right)^{2} d z .
\end{aligned}
$$


Define the vectors $V_{n \epsilon}=\left(V_{n, \epsilon, 1}, \ldots, V_{n, \epsilon, 2 k}\right), W_{\epsilon}=\left(W_{\epsilon, 1}, \ldots, W_{\epsilon, 2 k}\right), \xi_{n}=$ $\left(\xi_{n 1}, \ldots, \xi_{n 2 k}\right)$ and $\xi=\left(\xi_{1}, \ldots, \xi_{2 k}\right)$. Since by $(33)$ with probability at least $(1-\epsilon), K_{\epsilon}$ contains all $\tilde{D}_{n}^{h_{i}}$ eventually, we have $\mathbb{P}\left[V_{n \epsilon} \neq \xi_{n}\right]<\epsilon$ for all sufficiently large $n$. By (33) we similarly have $\mathbb{P}\left[W_{\epsilon} \neq \xi\right]<\epsilon$. Note, however, that by the continuous mapping theorem, for each fixed $\epsilon>0$, we have $V_{n \epsilon} \Rightarrow W_{\epsilon}$, as $n \rightarrow \infty$, because by Theorem 4.1 , the process $\left(X_{n}\left(., \theta_{i}\right), Y_{n}\left(., \theta_{i}\right): 1 \leq\right.$ $i \leq k)$ converges in distribution to $\left(\mathcal{S}_{a, b}()-.h_{i}, \mathcal{S}_{a, b}^{h_{i}}()-.h_{i}: 1 \leq i \leq\right.$ $k$ ) in $\left(L_{l o c}^{2}\right)^{2 k}$. We have thus, verified all the conditions of the converging together Lemma B.1 stated in the main paper and hence $\xi_{n} \Rightarrow \xi$, as $n \rightarrow \infty$. Finally in view of Proposition 4.1, by Slutsky's Theorem we have $f(n)\left(L_{n}\left(\theta_{0}+h d_{n}\right), T_{n}\left(\theta_{0}+h d_{n}\right)\right)$ converges to $\left(L_{\infty}(h), T_{\infty}(h)\right)$ in finite dimensional distribution.

Lemma 4.1. With probability one, the processes $L_{\infty}$ and $T_{\infty}$ as defined in (31) have continuous sample paths as functions of $h$.

Proof. We shall focus on $L_{\infty}$. The argument is similar for $T_{\infty}$. Define

$$
\begin{aligned}
\widetilde{\mathcal{S}}_{a, b}(z)= & \mathcal{L} \circ \mathcal{T}_{(\infty, 0]}(\mathbb{G}) \mathbf{1}_{(\infty, 0]}(z) \\
& +\mathcal{L} \circ \mathcal{T}_{(0, \infty)}(\mathbb{G}) \mathbf{1}_{(0, \infty)}(z),
\end{aligned}
$$

that is, on $(\infty, 0]\left((0, \infty)\right.$ respectively) $\widetilde{\mathcal{S}}_{a, b}(z)$ is the left derivative of the GCM of $\mathbb{G}$ restricted to $(\infty, 0]((0, \infty)$, resp.).

For any given $\epsilon>0$ we shall investigate the quantity

$$
\begin{aligned}
L_{\infty}(h+\epsilon)-L_{\infty}(h)= & \frac{1}{a^{2}} \int\left(\left(\mathcal{S}_{a, b}^{h}(z)\right)^{2}-\left(\mathcal{S}_{a, b}^{(h+\epsilon)}(z)\right)^{2}\right) d z \\
& -\frac{2 h}{a^{2}} \int\left(\mathcal{S}_{a, b}^{h}(z)-\mathcal{S}_{a, b}^{(h+\epsilon)}(z)\right) d z \\
& -\frac{2 \epsilon}{a^{2}} \int_{D_{a, b}^{h+\epsilon}}\left(\mathcal{S}_{a, b}(z)-\mathcal{S}_{a, b}^{(h+\epsilon)}(z)\right) d z
\end{aligned}
$$

Consider the following sets:

$$
\begin{aligned}
& A^{-}=\left\{z \in(-\infty, 0]: \widetilde{\mathcal{S}}_{a, b}(z)<h\right\} \\
& B^{-}=\left\{z \in(-\infty, 0]: h \leq \widetilde{\mathcal{S}}_{a, b}(z)<h+\epsilon\right\} \\
& C^{-}=\left\{z \in(-\infty, 0]: h+\epsilon \leq \widetilde{\mathcal{S}}_{a, b}(z)\right\} \\
& A^{+}=\left\{z \in(0, \infty): \widetilde{\mathcal{S}}_{a, b}(z)<h\right\} \\
& B^{+}=\left\{z \in(0, \infty): h \leq \widetilde{\mathcal{S}}_{a, b}(z)<h+\epsilon\right\} \\
& C^{+}=\left\{z \in(0, \infty): h+\epsilon \leq \widetilde{\mathcal{S}}_{a, b}(z)\right\}
\end{aligned}
$$


Now note that on $A^{-}$and $C^{+}$we have $\mathcal{S}_{a, b}^{h}(z)=\mathcal{S}_{a, b}^{h+\epsilon}(z)=\mathcal{S}_{a, b}^{c}(z)$. So the first two integrals of (36) are zero on these two sets. On $C^{-}$and $A^{+}$, we have $\mathcal{S}_{a, b}^{h}(z)=h$ and $\mathcal{S}_{a, b}^{h+\epsilon}(z)=(h+\epsilon)$. On $B^{-}, \mathcal{S}_{a, b}^{h}(z)=h$ and $\mathcal{S}_{a, b}^{h+\epsilon}(z)=\widetilde{\mathcal{S}}_{a, b}(z)<h+\epsilon$ and on $B^{+}, \mathcal{S}_{a, b}^{h}(z)=\widetilde{\mathcal{S}}_{a, b}(z)>h$ and $\mathcal{S}_{a, b}^{h+\epsilon}(z)=$ $h+\epsilon$. So on all these four sets $\mathcal{S}_{a, b}^{h}(z)$ and $\mathcal{S}_{a, b}^{h+\epsilon}(z)$ differ by at most $\epsilon$. As a result on these sets the integrand of the first integral in (36) is less than $\epsilon(\epsilon-h)$ and that of the second integral is less than $\epsilon$. Also notice that $\left(B^{-} \cup C^{-} \cup A^{+} \cup B^{+}\right) \subseteq\left(D_{a, b}^{h} \cup D_{a, b}^{h+\epsilon}\right)$ and by Theorem 3.1 from Supplement B for each $h, D_{a, b}^{h}$ is contained in a random compact interval with probability one. By monotonicity consideration it can be seen that, as $h$ increases the set $D_{a, b}^{h}$ shifts to the right. Therefore if $\left[L_{0}, U_{0}\right]$ and $\left[L_{1}, U_{1}\right]$ are compact intervals containing $D_{a, b}^{h}$ and $D_{a, b}^{h+1}$, respectively, for all $\epsilon \in(0,1)$, the set $D_{a, b}^{h} \cup D_{a, b}^{h+\epsilon}$ is contained in $\left[L_{0}, U_{1}\right]$. Hence we can make the first two integrals arbitrarily small by choosing small $\epsilon$. The third integral is also finite as again $D_{a, b}^{h+\epsilon}$ is subset of the compact interval $\left[L_{0}, U_{1}\right]$ and the integrand is bounded on the set they are being integrated on. So the difference $\left|L_{\infty}(h+\epsilon)-L_{\infty}(h)\right| \rightarrow 0$ as $\epsilon \rightarrow 0$. So $L_{\infty}(h)$ is continuous in $h$.

Lemma 4.2. The shape of $L_{\infty}$ and $T_{\infty}$ can be described as follows:

(i) $L_{\infty}\left(S_{a, b}(0)\right)=T_{\infty}\left(S_{a, b}(0)\right)=0$.

(ii) $L_{\infty}$ and $T_{\infty}$ have strictly decreasing sample paths on $\left(-\infty, S_{a, b}(0)\right]$ and strictly increasing sample paths on $\left(S_{a, b}(0), \infty\right)$.

Proof. Note that $S_{a, b}(z)=S_{a, b}^{S_{a, b}(0)}(z)$ for all $z \in \mathbb{R}$. This in view of (31) immediately proves (i). We shall prove (ii) only for $L_{\infty}$ and $h>S_{a, b}(0)$. The other cases can be treated similarly. Consider the process $\widetilde{\mathcal{S}}_{a, b}$ in (34). By definition (31), we can write

$$
\begin{aligned}
a^{2} L_{\infty}(h)= & \int_{\mathbb{R}}\left(\left(S_{a, b}(z)-h\right)^{2}-\left(S_{a, b}^{h}(z)-h\right)^{2}\right) d z \\
= & \int_{(-\infty, 0]}\left(\left(S_{a, b}(z)-h\right)^{2}-\left(S_{a, b}^{h}(z)-h\right)^{2}\right) d z \\
& +\int_{(0, \infty)}\left(\left(S_{a, b}(z)-h\right)^{2}-\left(S_{a, b}^{h}(z)-h\right)^{2}\right) d z \\
= & I_{1}(h)+I_{2}(h)
\end{aligned}
$$


Let $z_{1}<0<z_{2}$ be respectively the largest negative and smallest positive touch points of the process $\mathbb{G}$ with its GCM. Indeed by Lemma 2.1 from Supplement B these points exist. Observe that $\mathcal{T}_{(\infty, 0]}(\mathbb{G})(z)=\mathcal{T}(\mathbb{G})(z)$, for all $z \leq z_{1}$, that is the constrained and unconstrained GCMs coincide to the left of $z_{1}$. Therefore $\widetilde{\mathcal{S}}_{a, b}(z) \equiv \mathcal{S}_{a, b}(z), z \leq z_{1}$. Similarly $\widetilde{\mathcal{S}}_{a, b}(z) \equiv \mathcal{S}_{a, b}(z)$ for all $z \geq z_{2}$. Also by characterization of GCM it is easy to see that $\widetilde{\mathcal{S}}_{a, b}(z) \geq$ $\mathcal{S}_{a, b}(z)$ for $z \in\left(z_{1}, 0\right]$ and $\widetilde{\mathcal{S}}_{a, b}(z) \leq \mathcal{S}_{a, b}(z)$ for $z \in\left(0, z_{2}\right)$. Finally, note that by Lemma 2.3 of Supplement B, $\mathcal{S}_{a, b}(z) \mathcal{S}_{a, b}(0)=$ const on $z \in\left(z_{1}, z_{2}\right]$.

Now consider the first integral $I_{1}(h)$. As we have $h>_{a, b}(0)$, the integrand of $I_{1}(h)$ is zero outside the interval $\left(z_{1}, 0\right]$. Let $h_{1}>h_{2}$, then

$$
\begin{aligned}
& I_{1}\left(h_{1}\right)-I_{1}\left(h_{2}\right) \\
= & \int_{\left(z_{1}, 0\right]}\left(\left(\mathcal{S}_{a, b}(0)-h_{1}\right)^{2}-\left(\mathcal{S}_{a, b}(0)-h_{2}\right)^{2}\right) d z \\
& -\int_{\left(z_{1}, 0\right]}\left(\left(\widetilde{\mathcal{S}}_{a, b}(z) \wedge h_{1}-h_{1}\right)^{2}-\left(\left(\widetilde{\mathcal{S}}_{a, b}(z) \wedge h_{2}-h_{2}\right)^{2}\right) d z\right. \\
= & \int_{\left(z_{1}, 0\right]}\left(h_{1}+h_{2}-2 \mathcal{S}_{a, b}(0)\right)\left(h_{1}-H_{2}\right) d z- \\
& \int_{\left(z_{1}, 0\right]}\left(h_{1}-h_{2}-\widetilde{\mathcal{S}}_{a, b}(z) \wedge h_{1}+\widetilde{\mathcal{S}}_{a, b}(z) \wedge h_{2}\right) \\
& \left(h_{1}+h_{2}-\widetilde{\mathcal{S}}_{a, b}(z) \wedge h_{1}-\widetilde{\mathcal{S}}_{a, b}(z) \wedge h_{2}\right) d z .
\end{aligned}
$$

As $h_{1}>h_{2}$, we have $\widetilde{\mathcal{S}}_{a, b}(z) \wedge h_{1}-\widetilde{\mathcal{S}}_{a, b}(z) \wedge h_{2} \geq 0$. Therefore the second integrand is bounded above by $\left(h_{1}+h_{2} \widetilde{\mathcal{S}}_{a, b}(z) \wedge h_{1}-\widetilde{\mathcal{S}}_{a, b}(z) \wedge h_{2}\right)\left(h_{1} h_{2}\right)$. Hence we have,

$$
I_{1}\left(h_{1}\right)-I_{1}\left(h_{2}\right) \geq \int_{\left(z_{1}, 0\right]}\left(h_{1}-h_{2}\right)\left(\widetilde{\mathcal{S}}_{a, b}(z) \wedge h_{1}+\widetilde{\mathcal{S}}_{a, b}(z) \wedge h_{2} 2 \mathcal{S}_{a, b}(0)\right) d z
$$

which is non-negative because $h_{1}>h_{2}>\mathcal{S}_{a, b}(0)$ and $\widetilde{\mathcal{S}}_{a, b}(z)>\mathcal{S}_{a, b}(0)$ for $z \in\left(z_{1}, 0\right]$.

To show the monotonicity second integral $I_{2}(h)$, notice that if e $\widetilde{\mathcal{S}}_{a, b}(z)>$ $h$, then $z>z_{1}$ and $\mathcal{S}_{a, b}$ coincides with $\widetilde{\mathcal{S}}_{a, b}$, making the integrand of the second integral $I_{2}(h)$ zero. Moreover if $\widetilde{\mathcal{S}}_{a, b}(z) \leq h$, we have $\mathcal{S}_{a, b}^{h}(z)=h$. Therefore $I_{2}(h)$ can be written as $\int_{\widetilde{\mathcal{S}}_{a, b}(z) \leq h}\left(\mathcal{S}_{a, b}(0) h\right)^{2} d z$. As $h>\mathcal{S}_{a, b}(0)$, the second integral $I_{2}(h)$ is a strictly increasing function of $h$.

Finally as $I_{1}(h)$ is non-decreasing and $I_{2}(h)$ is strictly increasing in $h$, we have the strict monotonicity of $L_{\infty}(h)$ for $h>\mathcal{S}_{a, b}(0)$. 
Lemma 4.3. $\left\{f(n)\left(L_{n}\left(\theta_{0}+h d_{n}\right), T_{n}\left(\theta_{0}+h d_{n}\right)\right)\right\}_{h \in \mathbb{R}}$ as a process in $h$ is tight on $C(I) \times C(I)$ for any compact interval $I$, where $f(n)$ is defined in (32).

Proof. Note that by Theorem 4.2 and the Portmanteau Theorem we have $\limsup _{n \rightarrow \infty} \mathbb{P}\left(\left|f(n) L_{n}\left(\theta_{0}\right)\right| \geq a\right) \leq \mathbb{P}\left(\left|L_{\infty}(0)\right| \geq a\right) \rightarrow 0$ as $a \rightarrow \infty$. Now, let $I=[A, B]$ and $w_{x}(\delta)=\sup _{|s-t| \leq \delta}|x(s)-x(t)|$. By Theorem 3 of $\mathrm{AH}[2]$ and Corollary 1 of ZW[11], we have $d_{n}^{-1}\left(\hat{\theta}_{n}-\theta_{0}\right):=\Delta_{n}$ converges in distribution to some random variable (say, $\Delta$ ) and $L_{\infty}(\Delta)=0$. Also by Lemma 4.1, we have joint convergence of $\Delta_{n}$ and $f(n) L_{n}$.

Let $\epsilon>0$ and $\eta>0$ be given. By Lemma 4.1,

$$
\lim _{\delta \rightarrow 0} \mathbb{P}\left(w_{L_{\infty}}(\delta)>\epsilon / 2\right)=0 .
$$

So, choose $\delta_{1} \in(0,1 / 2)$ s.t., for all $0<\delta \leq \delta_{1}, \mathbb{P}\left(w_{L_{\infty}}(\delta)>\epsilon / 2\right)<\eta$. Now, take $\delta=2 \delta_{1}$. Take $A=h_{0}<h_{1}<\cdots<h_{k}=B$ with $h_{j}-h_{j-1}=\frac{\delta}{2}$ for $j=1,2, \ldots, k$. By the monotonicity property of $L_{n}\left(\theta_{0}+h d_{n}\right)$ to the left and right of $\Delta_{n}$, we have

$$
\begin{aligned}
& \limsup _{n \rightarrow \infty} \mathbb{P}\left(w_{f(n) L_{n}}(\delta) \geq \epsilon\right) \\
\leq & \limsup _{n \rightarrow \infty} \mathbb{P}\left(\max _{1 \leq j \leq k} f(n)\left|L_{n}\left(\theta_{0}+h_{j} d_{n}\right)-L_{n}\left(\theta_{0}+h_{j-1} d_{n}\right)\right| \mathbf{1}_{\Delta_{n} \in\left[t_{j-1}, t_{j}\right)^{c}}\right. \\
& \left.+\max \left\{f(n)\left|L_{n}\left(\theta_{0}+h_{j} d_{n}\right)\right|, f(n)\left|L_{n}\left(\theta_{0}+h_{j-1} d_{n}\right)\right|\right\} \mathbf{1}_{\Delta_{n} \in\left[t_{j-1}, t_{j}\right)} \geq \epsilon / 2\right)
\end{aligned}
$$

Now, since $\left(\Delta_{n},\left\{f(n) L_{n}\left(\theta_{0}+h d_{n}\right)\right\}_{h \in \mathbb{R}}\right) \Longrightarrow\left(\Delta,\left\{L_{\infty}(h)\right\}_{h \in \mathbb{R}}\right)$ the Portmanteau Theorem implies that the last limsup is bounded above by

$$
\begin{aligned}
& \mathbb{P}\left(\operatorname { m a x } _ { 1 \leq j \leq k } \left(\left|L_{\infty}\left(h_{j}\right)-L_{\infty}\left(h_{j-1}\right)\right| \mathbf{1}_{\Delta \in\left[t_{j-1}, t_{j}\right)^{c}}\right.\right. \\
& \left.\left.+\max \left\{\left|L_{\infty}\left(h_{j}\right)\right|,\left|L_{\infty}\left(h_{j-1}\right)\right|\right\} \mathbf{1}_{\Delta \in\left[t_{j-1}, t_{j}\right)}\right) \geq \epsilon / 2\right) \\
\leq & \mathbb{P}\left(w_{L_{\infty}}(\delta / 2) \geq \epsilon / 2\right)<\eta
\end{aligned}
$$

As $\eta>0$ is arbitrary, we have

$$
\lim _{\delta \rightarrow 0} \limsup _{n \rightarrow \infty} \mathbb{P}\left(w_{f(n) L_{n}}(\delta) \geq \epsilon\right)=0 .
$$

So, by Theorem 7.3 from Billingsley (1999)[5], we have $\left\{L_{n}\left(\theta_{0}+h d_{n}\right)\right\}_{h \in \mathbb{R}}$ is tight. Similar arguments yield the tightness of $\left\{T_{n}\left(\theta_{0}+h d_{n}\right)\right\}_{h \in \mathbb{R}}$ and the proof is complete.

Theorem 4.3. $\left\{f(n)\left(L_{n}\left(\theta_{0}+h d_{n}\right), T_{n}\left(\theta_{0}+h d_{n}\right)\right)\right\}_{h \in \mathbb{R}}$ converge in distribution to $\left\{\left(L_{\infty}(h), T_{\infty}(h)\right)\right\}_{h \in \mathbb{R}}$ uniformly in $C(I) \times C(I)$ where $f(n)$ is defined as in (32). 
Proof. Theorem 4.2 and Lemma 4.3 together imply the result.

Remark 4.2. If $\theta_{n}=\theta_{0}+a_{n}$ where $a_{n}=o\left(d_{n}\right)$, then $\left(L_{n}\left(\theta_{n}\right), T_{n}\left(\theta_{N}\right) \Rightarrow\right.$ $(\mathbb{L}, \mathbb{T})$.

Theorem 4.4. The length of the confidence intervals based on $L_{n}$ and $T_{n}$ decreases at a rate $d_{n}$, that is, if $\left(a_{n}, b_{n}\right)$ is the confidence interval, $b_{n}-a_{n}=$ $O_{p}\left(d_{n}\right)$ as $n \rightarrow \infty$.

Proof. By Theorem 4.3 we have

$$
f(n) L_{n}\left(\theta_{0}+h d_{n}\right) \Rightarrow L_{\infty}(h)
$$

uniformly on $C(I)$ for every compact interval $I$. Also by Lemma 3.2 the function $h \mapsto f(n) L_{n}\left(\theta_{0}+h d_{n}\right)$ is non-negative and it is minimized at $h=$ $H_{n}=d_{n}^{-1}\left(\hat{\theta}_{n}-\theta_{0}\right)$.

Let $\left(a_{n}, b_{n}\right)$ be the $L_{n}$-based confidence interval and $c_{0}$ be the cut-off. So we have

$$
\begin{aligned}
& d_{n}^{-1}\left(a_{n}-\theta_{0}\right)=\inf \left\{h: L_{n}\left(\theta_{0}+h d_{n}\right) \leq c_{0}\right\} \\
& d_{n}^{-1}\left(b_{n}-\theta_{0}\right)=\sup \left\{h: L_{n}\left(\theta_{0}+h d_{n}\right) \leq c_{0}\right\}
\end{aligned}
$$

Let $\mathcal{F}$ be the set of continuous real-valued U-shaped functions $f$ with minimum value 0 and $K: \mathcal{F} \rightarrow \mathbb{R}$ be the functional $K(g)=\inf \{h: g(h) \leq$ $\left.c_{0}\right\}$, with the convention $\inf \emptyset=-\infty$. Let $\left\{f_{n}\right\}_{n=1}^{\infty} \in \mathcal{F}$ be such that $f_{n} \rightarrow f$ uniformly on compact sets as $n \rightarrow \infty$. Also assume that $f \in \mathcal{F}$ is strictly decreasing before reaching the minimum and strictly increasing after that.Furthermore suppose that $f(x) \rightarrow \infty$ as $|x| \rightarrow \infty$. Note that by the continuity of $f_{n}$ and $f$ we have, $f(K(f))=f_{n}\left(K\left(f_{n}\right)\right)=c_{0}$. First let, $x_{0}$ be such that, $f\left(x_{0}\right)=0$ and $K(f)=x_{1}$. Let $0<\delta<x_{0}-x_{1}$. As $f_{n} \rightarrow f$ uniformly on $\left[x_{0}-\delta, x_{0}+\delta\right]$ we have $f_{n}\left(x_{0}-\delta\right) \rightarrow f\left(x_{0}-\delta\right)>0, f_{n}\left(x_{0}\right) \rightarrow f\left(x_{0}\right)=0$ and $f_{n}\left(x_{0}+\delta\right) \rightarrow f\left(x_{0}+\delta\right)>0$ as $n \rightarrow \infty$. So, there exists $N$ such that for all $n>N, f_{n}\left(x_{0}-\delta\right)>0$ and $f_{n}\left(x_{0}+\delta\right)>0$. So $f_{n}$ assumes value 0 at some point inside $\left(x_{0}-\delta, x_{0}+\delta\right)$ for all $n>N$. Now pick some $0<\epsilon<x_{0}-x_{1}-\delta$. As $f_{n} \rightarrow f$ uniformly on $\left[x_{1}-\epsilon, x_{1}+\epsilon\right]$ there exists $N_{1}>N$ such that for all $n>N_{1}$ we have, $f_{n}\left(x_{1}-\epsilon\right)<f\left(x_{1}\right)=c_{0}<f_{n}\left(x_{1}+\epsilon\right)$. So $K\left(f_{n}\right) \in\left(x_{1}-\epsilon, x_{1}+\epsilon\right)$ eventually. As $\epsilon>0$ can be chosen arbitrarily small we have $K\left(f_{n}\right) \rightarrow K(f)$ as $n \rightarrow \infty$.

Now, by Lemma 3.2 the function $h \mapsto L_{\infty}(h)$ is continuous, U-shaped, strictly decreasing on $\left(-\infty, S_{a, b}(0)\right]$ and strictly increasing on $\left[S_{a, b}(0), \infty\right)$. Therefore the functional $K$ is continuous at $L_{\infty}$ almost surely. So by continuous mapping theorem applied to $(37)$ we have $K\left(L_{n}\left(\theta_{0}+\dot{d}_{n}\right)\right)=d_{n}^{-1}\left(a_{n}-\right.$ 
$\left.\theta_{0}\right) \Rightarrow K\left(L_{\infty}\right)$ as $n \rightarrow \infty$. So, $d_{n}^{-1}\left(a_{n}-\theta_{0}\right)=O_{p}(1)$. Similarly we can show that $d_{n}^{-1}\left(b_{n}-\theta_{0}\right)=O_{p}(1)$. So the length of the confidence interval $\left(b_{n}-a_{n}\right)=O_{p}\left(d_{n}\right)$.

\section{Simulation Setup: Choosing bandwidth for esti- mating $m^{\prime}\left(t_{0}\right)$}

The bandwidth is chosen by the method of cross-validation. For this we divide the dataset in two parts randomly. Each data points were assigned to one of the two sets with probability 0.5 using an auxiliary Bernoulli(1/2) random variable. Let $D_{i}$ denote the set of indices for $i$-th subset, for $i=1,2$. Then for a given bandwidth $h$ we calculate $\hat{m}_{h, D_{i}}^{\prime}(t)$, the estimate of $m^{\prime}(t)$ based on set $D_{i}$ as

$$
\hat{m}_{h, D_{i}}^{\prime}(t)=\frac{1}{h} \int K\left(\frac{t-s}{h}\right) d \hat{m}_{D_{i}}(s)
$$

where $\hat{m}_{D_{i}}(t)$ is the MLE of $m(t)$ based on set $D_{i}$. We then numerically integrate $\hat{m}_{h, D_{i}}^{\prime}(t)$ to obtain $\hat{m}_{h, D_{i}}(t)$. Then we calculate

$$
C V(h)=\sum_{i \in D_{1}}\left(y_{i}-\hat{m}_{h, D_{2}}\left(t_{i}\right)\right)^{2}+\sum_{i \in D_{2}}\left(y_{i}-\hat{m}_{h, D_{1}}\left(t_{i}\right)\right)^{2} .
$$

Note that in calculating $C V$ we use the estimate based on one group to calculate the residual sum of square of the other group of the data set. We choose the value of $h$ that minimizes $C V(h)$ as optimal bandwidth.

\section{Assessing the performance of a kernel based method}

We used the kernel method described in Robinson (1997)[10] to construct confidence intervals and assess their performance in a limited simulation experiment. We considered the same two choices for $m(t)$ as previously, namely:

$m_{1}(t)=e^{t} \quad$ and $\quad m_{2}(t)= \begin{cases}t, & t \in(0,1 / 4] \\ 1 / 4+20000(t-1 / 4)^{2}, & t \in(1 / 4,1 / 4+1 / 200] \\ t+3 / 4, & t \in(1 / 4+1 / 200,1]\end{cases}$

with $t_{0}=1 / 2$ and $t_{0}=1 / 4+1 / 400$ respectively. For SRD we used an $\operatorname{AR}(2)$ process with coefficients 0.7 and -0.6 and for LRD, fractional Gaussian noise 
Table 7: Coverage of $90 \%$ Confidence Intervals constructed using a Kernel Method

\begin{tabular}{|l|l|c|c|}
\hline Function & \multicolumn{1}{|c|}{ Errors } & $\mathrm{n}$ & Coverage \\
\hline$m_{1}(t)$ & $\mathrm{AR}(2)$ coeff $(0.7,-0.6)$ & 500 & $91.7 \%$ \\
\hline$m_{1}(t)$ & $\mathrm{AR}(2)$ coeff $(0.7,-0.6)$ & 1000 & $92.9 \%$ \\
\hline$m_{1}(t)$ & fGn, $\mathrm{H}=0.9$ & 500 & $91.4 \%$ \\
\hline$m_{1}(t)$ & fGn, $\mathrm{H}=0.9$ & 1000 & $90.8 \%$ \\
\hline$m_{2}(t)$ & $\mathrm{AR}(2)$ coeff $(0.7,-0.6)$ & 500 & $80.6 \%$ \\
\hline$m_{2}(t)$ & $\mathrm{AR}(2)$ coeff $(0.7,-0.6)$ & 1000 & $82.5 \%$ \\
\hline$m_{2}(t)$ & fGn, $\mathrm{H}=0.9$ & 500 & $52.8 \%$ \\
\hline$m_{2}(t)$ & fGn, $\mathrm{H}=0.9$ & 1000 & $60.1 \%$ \\
\hline
\end{tabular}

with $\mathrm{H}=0.9$. The marginal variance of the error was 0.2 as in our previous simulations. Some of the details of the implementation (including choice of kernels and bandwidths) are provided in Section 6.3 of the main paper. It is evident from Table 7 that this method depends heavily on the structure of the regression function. It works well for a smooth function but the coverage drops drastically, especially under long range dependence, if the trend function is not well-behaved.

Bandwidth choice is of course an issue as with all smoothing procedures and a good bandwidth choice could potentially improve performance. The optimal bandwidth can be theoretically derived but involves estimating the second derivative of the function. We have not pursued this in our simulations.

\section{Some Auxiliary Lemmas}

In this section we state some Lemmas useful for our purpose.

Lemma 7.1. Let $f_{n}, f$ be convex functions, defined on an open interval $I \subset \mathbb{R}$. If $\lim _{n \rightarrow \infty} \sup _{x \in I}\left|f_{n}(x)-f(x)\right|=0$, then, for all $x \in I$,

$$
\partial_{\ell} f(x) \leq \liminf _{n \rightarrow \infty} \partial_{\ell} f_{n}(x) \leq \limsup _{n \rightarrow \infty} \partial_{r} f_{n}(x) \leq \partial_{r} f(x),
$$

where $\partial_{\ell} f$ and $\partial_{r} f$ denote the left and right derivatives of $f$. If, moreover, the function $f$ is differentiable at a point $x$ with derivative $f^{\prime}(x)$, then both $\partial_{\ell} f_{n}(x)$ and $\partial_{r} f_{n}(x)$ converge to $f^{\prime}(x)$, as $n \rightarrow \infty$.

For the proof, see e.g. p. 330 in Robertson et. al. (1988)[9]. 
Lemma 7.2. Let $f_{n}, f$ be convex functions, defined on an open interval $I \subset \mathbb{R}$. If $f_{n} \rightarrow f$, as $n \rightarrow \infty$ uniformly on all compact subsets of $I$, then $\partial_{\ell} f_{n} \rightarrow \partial_{\ell} f$ in $L_{l o c}^{2}$.

Proof. Since $f$ is convex it is a.e. differentiable and by Lemma 7.1, we have $\partial_{\ell} f_{n}(z) \rightarrow \partial_{\ell} f(z) \equiv \partial_{r} f(z)$, as $n \rightarrow \infty$, for almost all $z \in I$.

Now, for any given $[c, d] \subset I$, one can find $a \leq c<d \leq b$, such that $[a, b] \subset I$ and $f$ is differentiable at both $a$ and $b$. Thus, by Lemma 7.1 $\partial_{\ell} f_{n}(x) \rightarrow \partial_{\ell} f(x), x \in\{a, b\}$ as $n \rightarrow \infty$. Since $\partial_{\ell} f_{n}:[a, b] \rightarrow \mathbb{R}$, is nondecreasing, we have $\partial_{\ell} f_{n}(a) \leq \partial_{\ell} f_{n}(z) \leq \partial_{\ell} f_{n}(b), z \in[a, b]$, and by the fact that the last lower and upper bounds converge, we have

$$
\sup _{z \in[a, b]}\left|\partial_{\ell} f_{n}(z)\right| \leq 1+\max \left\{\left|\partial_{\ell} f(a)\right|,\left|\partial_{\ell} f(b)\right|\right\}<\infty,
$$

for all sufficiently large $n$. Therefore, by the dominated convergence theorem

$$
\int_{[a, b]}\left(\partial_{\ell} f_{n}(z)-\partial_{\ell} f(z)\right)^{2} d z \rightarrow 0, \quad \text { as } n \rightarrow \infty,
$$

which completes the proof.

Lemma 7.3. Let $M(I)$ denote the set of monotone non-decreasing and left-continuous functions defined on the interval I equipped with the $L_{l o c}^{2}$ convergence. Define the concatenation map $C_{h}: M(-\infty, 0) \times M(0, \infty) \rightarrow$ $M(-\infty, \infty)$, where

$$
C_{h}(f, g)(x):= \begin{cases}f(x) \wedge h & , \text { if } x \in(-\infty, 0) \\ \lim _{u \uparrow \uparrow} f(u) \wedge h & , \text { if } x=0 \\ g(x) \vee h & , \text { if } x \in(0, \infty)\end{cases}
$$

Then, $C_{h}:\left(M(-\infty, 0) \times M(0, \infty), L_{l o c}^{2} \times L_{l o c}^{2}\right) \rightarrow\left(M(-\infty, \infty), L_{l o c}^{2}\right)$ is continuous.

Proof. Let $f_{n} \rightarrow f$ and $g_{n} \rightarrow g$ in $\left(M(-\infty, 0), L_{l o c}^{2}\right)$ and $\left(M(0, \infty), L_{l o c}^{2}\right)$ respectively and let $a<0<b$. It is enough to show that $\int_{[a, 0]}\left(f_{n}(x) \wedge \theta-\right.$ $f(x) \wedge \theta)^{2} d x \rightarrow 0$ and that $\int_{[0, b]}\left(g_{n}(x) \vee \theta-g(x) \vee \theta\right)^{2} d x \rightarrow 0, n \rightarrow \infty$. We only focus on the first integral since the second one can be treated similarly.

Observe that $\left(f_{n}(x) \wedge \theta-f(x) \wedge \theta\right)^{2} \leq\left(f_{n}(x)-f(x)\right)^{2}$. Therefore by the fact that $f_{n} \rightarrow f$ in $L_{l o c}^{2}(-\infty, 0)$, it follows that it is enough to show that

$$
\lim _{\epsilon \rightarrow 0} \lim _{n \rightarrow \infty} \int_{[-\epsilon, 0]}\left(f_{n}(x) \wedge \theta-f(x) \wedge \theta\right)^{2} d x=0 .
$$


Observe that by the monotonicity of $f_{n}$, we have

$$
\left|f_{n}(x) \wedge \theta\right| \leq \max \left\{|\theta|,\left|f_{n}(x-1)\right|\right\}, \quad \text { for all } x \in[-1,0] \text {. }
$$

Therefore, using the inequality $(u-v)^{2} \leq 2 u^{2}+2 v^{2}$, we get

$$
\int_{[-\epsilon, 0]}\left(f_{n}(x) \wedge \theta-f(x) \wedge \theta\right)^{2} d x \leq 2 \int_{[-\epsilon-1,-1]}\left(f_{n}^{2}(x)+f^{2}(x)\right) d x+4|\theta|^{2} \epsilon
$$

Since $f_{n} \rightarrow f$ in $L_{l o c}^{2}(-\infty, 0)$, we get $\int_{[-\epsilon-1,-1]} f_{n}^{2}(x) d x \rightarrow \int_{[-\epsilon-1,-1]} f^{2}(x) d x$, and the latter vanishes as $\epsilon \downarrow 0$. Therefore, the right-hand side of (40) vanishes as $n \rightarrow \infty$ and as $\epsilon \downarrow 0$, which implies (39).

\section{References}

[1] Adler, R. J. and Taylor, J. E.(2005) Random Fields and Geometry. Springer, New York.

[2] Anevski, D. and Hössjer, O.(2006) A general asymptotic scheme for inference under order restrictions. The Annals of Statistics Vol. 34, 18741930 .

[3] Banerjee, M.(2000) Likelihood Ratio Inference in Regular and Nonregular Problems. PhD Thesis. University of Washington.

[4] Banerjee, M. and Wellner, J. A.(2001) Likelihood ratio tests for monotone functions. The Annals of Statistics Vol. 29, 1699-1731.

[5] Billingsley, P.(1999) Convergence of Probability Measures. New York: Wiley.

[6] Bingham, N. H. (1971) Limit Theorems for Occupation Times of Markov Processes. Z. Wahrscheinlichkeitstheorie Vol 17, 1-22.

[7] Brunk, H. D. (1970) Estimation of isotonic regression. In Nonparametric Techniques in Statistical Inference (M. L. Puri, ed,) 177-197. Cambridge University Press, London.

[8] Groeneboom, P. (1989). Brownian motion with a parabolic drift and airy function. Probability Theory and Related Fields Vol 81, 79-109.

[9] Robertson, T., Wright, F. T. and Dykstra, R. L.(1988). Order Restricted Statistical Inference. New York: Wiley. 
[10] Robinson, P. M. (1997). Large-sample inference for nonparametric regression with dependent errors. The Annals of Statistics, Vol. 25 no. 5, 2054-2083.

[11] Zhao, O. and Woodroofe, M.(2012) Estimating a monotone trend. Statistica Sinica, Vol. 22, 359-378. 


\title{
Behavior of GCM of Brownian and fractional Brownian motion with a quadratic drift and Related Statistics
}

\author{
Pramita Bagchi, Moulinath Banerjee, and Stilian A. Stoev
}

July 2,2018

\begin{abstract}
Here we establish some important properties of GCM type functionals of fractional Brownian motion with a quadration drift, which are essential to prove the results discussed in the main paper under long range dependence. We also discuss some propeties of the random variables $\mathbb{L}, \mathbb{T}$ and $\Psi$ appearing in the limit distributions of the test statistics, we have considered in our main paper.
\end{abstract}

\section{Introduction and Problem Formulation}

Functionals of Brownian and fractional Brownian motion with a quadratic drift play an important role in shape restricted regression. The normalized least squares estimator in the isotonic regression problem with iid data converges in law to Chernoff's distribution, which is the minimizer of a Brownian motion with a quadratic drift (Brunk, 1970 [6]). This minimizer can be characterized in terms of the greatest convex minorant (GCM) of the process: it is a multiple of the slope of the GCM at 0. GCM-type functionals of Brownian motion and properties of Chernoff's distribution are well studied (Groeneboom 1983[8], 1989[9], 2011[10]). However, inference using the least squares estimator involves estimating a nuisance parameter, the slope of the regression function, which is hard to estimate. Banerjee and Wellner (2001 [3], 2005[4]), Banerjee (2009)[5] developed a likelihood ratio/residual sum of squares based test for this isotonic regression problem which is asymptotically pivotal: it has a parameter-free 'universal' limit distribution which involves integral type functionals of GCMs of quadratically drifted Brownian motion. 
AH (2006)[2] and ZW(2012)[17] studied the least squares estimator for isotonic regression under dependence. For weak dependence, the limit results remain the same as in the iid case while for strong dependence, the limit distribution turns out to be the minimizer of fractional Brownian motion with a quadratic drift. In our main paper we study the limit behavior of residual sum of squares (type) statistics under dependence, which gives rise to integral type functionals of the GCM of quadratically drifted fractional Brownian motion (fBM). The properties of these GCMs were partially studied by $\mathrm{AH}(2006)[2]$. In this technical report, we develop some further properties of these GCMs and establish several important properties of the integral type functionals that describe the limiting distributions of the statistics proposed in the main paper. This is required to theoretically validate the statistical methodology but can also be of independent interest.

Mathematically we denote a two-sided fBm by $\left\{B_{H}(t)\right\}_{t \in \mathbb{R}}$ where $1 / 2 \leq$ $H \leq 1$, with $B_{1 / 2}(t)$ denoting the Brownian motion case. Define the process $\mathbb{G}_{a, b}(z):=a B_{H}(z)+b z^{2}$ for $z \in(-\infty, \infty)$. For a $f: \mathbb{R} \rightarrow \mathbb{R}$, we define $T_{I}(f)$ to be the GCM of $f$ restricted to the interval $I$. If $I=\mathbb{R}$ we drop the subscript. As discussed in (12) in the main paper, we consider the following process

$$
\begin{aligned}
& \mathcal{S}_{a, b}(z)=\mathcal{L} \circ \mathcal{T}(\mathbb{G})(z) \\
& \mathcal{S}_{a, b}^{h}(z)= \begin{cases}\mathcal{L} \circ \mathcal{T}_{(-\infty, 0)}(\mathbb{G})(z) \wedge h & , z \in(-\infty, 0) \\
\lim _{u \uparrow 0} \mathcal{L} \circ \mathcal{T}_{(-\infty, 0)}(\mathbb{G})(u) \wedge h & , z=0 \\
\mathcal{L} \circ \mathcal{T}_{(0, \infty)}(\mathbb{G})(z) \vee h & , z \in(0, \infty)\end{cases}
\end{aligned}
$$

and the random variables (as (13) from the main paper)

$\mathbb{L}_{a, b}=\int_{\mathbb{R}}\left(\left(\mathcal{S}_{a, b}(z)\right)^{2}-\left(\mathcal{S}_{a, b}^{0}(z)\right)^{2}\right) d z$ and $\mathbb{T}_{a, b}=\int_{\mathbb{R}}\left(\mathcal{S}_{a, b}(z)-\mathcal{S}_{a, b}^{0}(z)\right)^{2} d z$

Specifically, we establish useful properties of $\mathbb{L}_{a, b}$ and $\mathbb{T}_{a, b}$. When $a=1$ and $b=1$, we drop the subscript for convenience. Furthemore, in the long range dependence case, we use another pivotal statistic $\Psi$, which is a function of the ratio of $\mathbb{L}$ to $\mathbb{T}$, for inference. We discuss the simulation of quantiles of both $\mathbb{L}$ and $\Psi$ and their empirical behavior. 


\section{Properties of GCM of a Function}

Before discussing the properties of functionals of Brownian and fractional Brownian motion, we provide some technical lemmas about GCMs of deterministic functions. For a real-valued function $f$ on $\mathbb{R}$, introduce the constrained GCM:

$$
\mathcal{T}_{0}(f)(z)= \begin{cases}\mathcal{T}_{(0, \infty)}(f)(z) & , \text { if } z \in(0, \infty) \\ \mathcal{T}_{(-\infty, 0]}(f)(z) & , \text { if } z \in(-\infty, 0]\end{cases}
$$

Lemma 2.1. Let $f: \mathbb{R} \rightarrow \mathbb{R}$ be a continuous function such that $f(t) /|t| \rightarrow \infty$ as $|t| \rightarrow \infty$. Then, there exist points $t_{1}<0$ and $t_{2}>0$, such that $\mathcal{T}(f)\left(t_{i}\right)=$ $f\left(t_{i}\right), i=1,2$.

Proof. Observe that if $\phi$ is a convex minorant of $f$ such that $f(t)=\phi(t)$, then $\mathcal{T}(f)(t)=f(t)$. We will construct such a $\phi$ with $f\left(t_{2}\right)=\phi\left(t_{2}\right), t_{2}>0$. The existence of a negative touch point can be treated similarly.

Note that $f$ is bounded below since $f(t) \rightarrow \infty,|t| \rightarrow \infty$ and let $a:=$ $\inf _{t \in \mathbb{R}} f(t)>-\infty$. If this infimum is attained at $t_{2}>0$, then $\phi(t):=a, t \in \mathbb{R}$ is the desired convex minorant. Otherwise, let $\ell(t):=\lambda t+a$ be a line with positive slope $\lambda>0$, that passes through the points $(0, a)$ and $(x, f(x))$, for some $x>0$. Such a line exists since $f(x) \rightarrow \infty$. We consider two cases:

(i) If $\ell(t) \leq f(t)$, for all $t \geq 0$, then the convex minorant $\phi(t):=$ $a 1_{(-\infty, 0]}(t)+\ell(t) 1_{(0, \infty)}(t), t \in \mathbb{R}$ has the desired property with $t_{2}:=x$.

(ii) If $\ell(t)>f(t)$, for some $t \geq 0$, then the infimum $b:=\inf _{t \geq 0}(f(t)-$ $\ell(t))<0$ is negative, and it is attained at some $t_{2}>0$, because $f$ is continuous and $f(t) / t \rightarrow \infty, t \rightarrow \infty$. In this case, $\phi(t):=a 1_{(-\infty,|b| / \lambda]}(t)+(\ell(t)+$ b) $1_{(|b| / \lambda, \infty)}(t), t \in \mathbb{R}$ is a convex minorant for $f$ with $\phi\left(t_{2}\right)=f\left(t_{2}\right)$. This completes the proof.

Lemma 2.2. For any continuous real-valued function $f$ on $\mathbb{R}$ and $\mathcal{T}_{0}$ as in (3), we have:

(i) $\mathcal{T}(f)(t) \leq \mathcal{T}_{0}(f)(t) \leq f(t), t \in \mathbb{R}$.

(ii) If $f\left(t_{0}\right)=\mathcal{T}(f)\left(t_{0}\right)$ for some $t_{0}>0$ ( $t_{0}<0$, respectively) then $\mathcal{T}(f)(t)=\mathcal{T}_{0}(f)(t)$ for all $t \geq t_{0}\left(t \leq t_{0}\right.$, respectively $)$.

Proof. The first assertion is immediate by definition since the constrained GCM is no greater than the unconstrained one. To prove the second statement, suppose $\mathcal{T}_{0}(f)\left(t_{0}\right)=\mathcal{T}(f)\left(t_{0}\right)$ for some $t_{0}>0$. Let $\phi(t)=\mathcal{T}(f)(t) \mathbf{1}_{\left(-\infty, t_{0}\right]}(t)+$ $\mathcal{T}_{0}(f)(t) \mathbf{1}_{\left(t_{0}, \infty\right)}(t)$. By the already established part $(i)$, we have that $\mathcal{T}(f)(t) \leq$ $\mathcal{T}_{0}(f)(t) \leq f(t)$, and hence one can show that the function $\phi$ is convex and it 
is also a minorant of $f$. Therefore, $\phi(t) \equiv \mathcal{T}_{0}(f)(t) \leq \mathcal{T}(f)(t)$ for all $t \geq t_{0}$, which in view of part (i) yields $\mathcal{T}(f)(t)=\mathcal{T}_{0}(f)(t), t \geq t_{0}$. The case $t_{0}<0$ can be treated similarly.

Lemma 2.3. Let $f$ be a continuous function with $f(0)=0, f(t) /|t| \rightarrow \infty$ as $|t| \rightarrow \infty$ and such that both $\inf _{(-\infty, 0)} f(t)$ and $\inf _{(0, \infty)} f(t)$ are negative. Then we have the following:

(i) There exist $x_{*}<0<x^{*}$, such that the GCM of $f$ coincides with the line joining $\left(x_{*}, f\left(x_{*}\right)\right)$ and $\left(x^{*}, f\left(x^{*}\right)\right)$ on the interval $\left[x_{*}, x^{*}\right]$.

(ii) Moreover, $\mathcal{L} \circ \mathcal{T}(f)(0)=0$ implies that $\inf _{t \in(-\infty, 0]} f(t)=\inf _{t \in[0, \infty)} f(t)$.

Proof. Let $C=\{x \in \mathbb{R}: f(x)=\mathcal{T}(f)(x)\}$. First note that $C$ is a closed set since $f$ and $\mathcal{T}(f)$ are both continuous. By Lemma 2.1 there exist a positive point and a negative point in $C$. Observe that by the convexity of $\mathcal{T}(f)$ and the fact $\inf _{(-\infty, 0)} f(t)$ and $\inf _{(0, \infty)} f(t)$ are negative, the point 0 does not belong to $C$. Indeed, no convex function passing through the origin could take strictly negative values on both $(-\infty, 0)$ and $(0, \infty)$.

Define $x_{*}:=\sup \{x \in C, x<0\}$ and $x^{*}:=\inf \{x \in C, x>0\}$. Since $C$ is closed we have that $x_{*}, x^{*} \in C$, also $\left(x_{*}, x^{*}\right) \cap C=\emptyset$ and $x_{*}<0<x^{*}$, since $0 \notin C$. To prove $(i)$, consider the function

$$
\widetilde{\mathcal{T}}(x):= \begin{cases}\mathcal{T}(f)(x) & , \text { if } x \notin\left[x_{*}, x^{*}\right] \\ \ell(x) & , \text { if } x \in\left[x_{*}, x^{*}\right]\end{cases}
$$

where $\ell(x)=\left(f\left(x^{*}\right)-f\left(x_{*}\right)\right)\left(x-x_{*}\right) /\left(x^{*}-x_{*}\right)+f\left(x_{*}\right)$ is the equation of the line joining the points $\left(x_{*}, f\left(x_{*}\right)\right)$ and $\left(x^{*}, f\left(x^{*}\right)\right)$. We will show that $\widetilde{\mathcal{T}}$ coincides with $\mathcal{T}(f)$. Since $\mathcal{T}(f)$ is convex and its graph passes through the points $\left(x_{*}, f\left(x_{*}\right)\right)$ and $\left(x^{*}, f\left(x^{*}\right)\right)$, it follows that $\widetilde{\mathcal{T}}$ is convex, and in fact $\widetilde{\mathcal{T}}(x) \geq \mathcal{T}(f)(x), x \in \mathbb{R}$. Therefore, it remains to show that $\widetilde{\mathcal{T}}$ is a minorant of $f$, which amounts to proving $\widetilde{\mathcal{T}}(x) \equiv \ell(x) \leq f(x), x \in\left[x_{*}, x^{*}\right]$.

Suppose that $\min _{x \in\left[x_{*}, x^{*}\right]}(f(x)-\ell(x))<0$, which by continuity is attained at some $x_{0} \in\left(x_{*}, x^{*}\right)$. Define the linear function $\widetilde{\ell}(x):=\ell(x)+$ $\left(f\left(x_{0}\right)-\ell\left(x_{0}\right)\right)$ and observe that $\widetilde{\ell}(x)<\ell(x), x \in \mathbb{R}$ and hence $\widetilde{\ell}$ is a (trivial) convex minorant to $f$. We also have that $\widetilde{\ell}\left(x_{0}\right)=f\left(x_{0}\right)$, which implies that $x_{0} \in C$. This is however a contracticiton since $x_{0} \in\left(x_{*}, x^{*}\right)$, and as argued above $\left(x_{*}, x^{*}\right) \cap C=\emptyset$.

We now prove (ii). Suppose that the slope of GCM on $\left[x_{*}, x^{*}\right]$ is 0 . Then, by part (i), the horizontal line passing through the points $\left(x_{*}, f\left(x_{*}\right)\right)$ and $\left(x^{*}, f\left(x^{*}\right)\right)$ is a convex minorant of $f$ over the entire real line, which touches the graph of the function at $x_{*}$ and $x^{*}$. This shows that the infima of $f$ on 
the intervals $(-\infty, 0)$ and $(0, \infty)$ are equal and are attained at $x_{*}$ and $x^{*}$, respectively.

Lemma 2.4. Assume that $f$ is continuous, $f(0)=0$, $\inf _{t \in(0, \infty)} f(t)<0$, and $f(t) \rightarrow \infty$ as $t \rightarrow \infty$. Then $\lim _{\delta \downarrow} \mathcal{T}_{[-\delta, \infty)}(f)(0)=0$.

Proof. First note that for each fixed $t, \mathcal{T}_{[-\delta, \infty)}(f)(t)$ is a non-increasing function of $\delta>0$ and define

$$
\varphi(t):=\lim _{\delta \downarrow 0} \mathcal{T}_{[-\delta, \infty)}(f)(t) \equiv \sup _{\delta>0} \mathcal{T}_{[-\delta, \infty)}(f)(t) .
$$

The function $\varphi$ is a convex minorant of $f$ on $[0, \infty)$. We will prove the result by contradiction. Indeed, assume that $\lim _{\delta \downarrow 0} \mathcal{T}_{[-\delta, \infty)}(f)(0)=\varphi(0)=:-a<$ 0 . Since $f$ is continuous and $f(t) \rightarrow \infty$, as $t \rightarrow \infty$, we have $\inf _{[0, \infty)} f(t)=$ $f\left(t^{*}\right)$, for some point $t^{*} \geq 0$. Since also $f(0)=0>\inf _{t \in(0, \infty)} f(t)=f\left(t^{*}\right)$, it follows that $t^{*}>0$. The continuity of $f$ and the fact that $f(0)=0$, imply that $\exists \delta_{0}>0$ small enough such that $f(t) \geq f\left(t_{*}\right)$ for all $t \in\left[-\delta_{0}, \infty\right)$. Therefore, the horizontal line passing through $\left(t_{*}, f\left(t_{*}\right)\right)$ is a minorant of $f$ in the interval $\left[-\delta_{0}, \infty\right)$. This shows that $t_{*}$ is a touch point of $\mathcal{T}_{[-\delta, \infty)}(f)$ and $f$ for $0 \leq \delta \leq \delta_{0}$ and hence it is a touch point of $\varphi$ and $f$, too. Also, $\mathcal{T}_{[-\delta, \infty)}(f)$, for $0 \leq \delta \leq \delta_{0}$ and consequently $\varphi$ lie above the horizontal line mentioned above. As a result both convex minorants $\mathcal{T}_{[0, \infty)}(f)$ and $\varphi$ are non-increasing in the interval $\left[0, t_{*}\right]$.

Consider now the convex set $C=\left\{\vec{x}=\left(x_{1}, x_{2}\right) \in \mathbb{R}^{2}: 0 \leq x_{1} \leq\right.$ $\left.t_{*}, \mathcal{T}_{[0, \infty)}(f)\left(x_{1}\right) \leq x_{2} \leq 0\right\}$. Let $d=\inf _{\vec{x} \in C}\|\vec{x}-\vec{v}\|$ be the distance from the point $\vec{v}:=(0,-a)$ to the set $C$. Define the $d$-neighborhood of $C, C_{d}=\{\vec{y} \in$ $\left.\mathbb{R}^{2}: \inf _{\vec{x} \in C}\|\vec{y}-\vec{x}\| \leq d\right\}$. Note that $C_{d}$ is also a closed convex set and $\vec{v}$ is a point on its boundary. Thus, by the characterization of convex sets there exists a vector to $\vec{\Delta}=\left(\Delta_{1}, \Delta_{2}\right)$, such that $\langle\vec{u}-\vec{v}, \vec{\Delta}\rangle>0$, for all interior points $\vec{u}$ of $C_{d}$.

We will show that both components $\Delta_{1}$ and $\Delta_{2}$ of $\vec{\Delta}$ are positive. Indeed, since $\overrightarrow{0}$ is an interior point of $C_{d}$, we have $\langle\overrightarrow{0}-\vec{v}, \vec{\Delta}\rangle=\Delta_{2} a>0$, which implies $\Delta_{2}>0$. Also, considering the interior point $\left(t_{*}, f\left(t_{*}\right)\right)$ of $C_{d}$, we get $\left\langle\left(t_{*}, f\left(t_{*}\right)\right)-(0, \varphi(0)), \vec{\Delta}\right\rangle=t_{*} \Delta_{1}+\left(f\left(t_{*}\right)-\varphi(0)\right) \Delta_{2}>0$, which in turn implies $t_{*} \Delta_{1}>\left(\varphi(0)-f\left(t_{*}\right)\right) \Delta_{2}$. The last quantity is positive since $f\left(t_{*}\right)=\varphi\left(t_{*}\right)<\varphi(0)$. This yields $\Delta_{1}>0$.

Note that the compact set $K:=\left\{(t, f(t)): t \in\left[0, t_{*}\right]\right\}$ is contained in the interior of $C_{d}$. Therefore,

$$
\inf _{t \in\left[0, t_{*}\right]}\langle\vec{\Delta},(t, f(t))-(0, \varphi(0))\rangle=\inf _{t \in\left[0, t_{*}\right]}\left(\Delta_{1} t+\Delta_{2}(f(t)-\varphi(0))\right):=c>0 .
$$


This means that the linear function $\ell(t):=-\left(\Delta_{1} / \Delta_{2}\right) t+c / 2 \Delta_{2}+\varphi(0)$ is a minorant of the function $f$ on the interval $\left[0, t_{*}\right]$. Since also the minimum of $f$ on $[0, \infty)$ is attained at $t_{*}$ and the slope of this aforementioned line is negative, the function $\ell(t)$ is also a minorant of $f$ on $[0, \infty)$.

By the continuity of $f$ at 0 , the above line is a minorant of $f$ on $[-\delta, \infty)$ for some $\delta>0$ and it passes through the point $\left(0, \varphi(0)+c / 2 \Delta_{2}\right)$. This, however, contradicts the fact that any such minorant should be no greater than $\mathcal{T}_{[-\delta, \infty)}(f)(0) \leq \varphi(0)$ at 0 .

\section{Properties of the GCM-type functionals of the fractional Brownian motion plus quadratic drift}

Let now $\left\{B_{H}(t)\right\}_{t \in \mathbb{R}}$ denote the two-sided fractional Brownian motion with Hurst index $H \in[1 / 2,1)$. These processes have versions with continuous paths and in the sequel we shall work with such versions. Note that if $H=1 / 2$, then $B_{H}$ is the usual Brownian motion involved in the limit of under short range dependence. For $1 / 2<H<1$ it is two-sided version of fBm with Hurst index $H$. Define

$$
\mathbb{G}_{a, b}^{H}(t)=a B_{H}(t)+b t^{2}, \quad a, b>0 .
$$

By using the self similarity of the $\mathrm{fBm}$ one can show that

$$
\left\{\mathbb{G}_{a, b}^{H}(z)\right\}_{z \in \mathbb{R}} \stackrel{d}{=} a(a / b)^{\frac{H}{2-H}}\left\{\left(\mathbb{G}_{1,1}^{H}\left((b / a)^{\frac{1}{2-H}} z\right)\right)\right\}_{z \in \mathbb{R}} .
$$

Thus in what follows it suffices to focus on the standardized process

$$
\xi_{H}(t):=\mathbb{G}_{1,1}(t)=B_{H}(t)+t^{2}, t \in \mathbb{R} .
$$

We shall establish certain properties of the process $\xi_{H}$, its GCM $\mathcal{T}\left(\xi_{H}\right)$ and its constrained counterpart $\mathcal{T}_{0}\left(\xi_{H}\right)$. All results established below for $\xi_{H}$ and its functionals extend immediately to $\mathbb{G}_{a, b}^{H}$ (and its functionals) in light of (4).

Lemma 3.1. With probability one, we have $B_{H}(t) / t \rightarrow 0$, as $|t| \rightarrow \infty$.

Proof. Define $X_{k}=\sup _{t \in[k-1, k]}\left|B_{H}(t)\right|$. By self-similarity we then have $X_{k} \stackrel{d}{=} k^{H} \sup _{t \in\left[\frac{k-1}{k}, 1\right]}\left|B_{H}(t)\right|$. By the Borell inequality (Adler and Taylor, 2005[1]), for some $c>0$ and all $\epsilon>0$,

$$
\mathbb{P}\left(X_{k}>\epsilon(k-1)\right) \leq \mathbb{P}\left(\sup _{t \in\left[\frac{k-1}{k}, 1\right]}\left|B_{H}(t)\right|>\frac{\epsilon(k-1)}{k^{H}}\right) \leq e^{-c \frac{(k-1)^{2}}{k^{2 H}}} .
$$


This implies that, $\sum_{k=1}^{\infty} \mathbb{P}\left(X_{k} /(k-1)>\epsilon\right)<\infty$, and by the Borel-Cantelli Lemma, $\mathbb{P}\left(X_{k} /(k-1)>\epsilon i . o\right.$. $)=0$. Consequently, $\sup _{j \geq k} X_{j} /(j-1) \rightarrow 0$ a.s. as $k \rightarrow \infty$. Thus, $\sup _{t>k}\left|B_{H}(t) / t\right| \leq \sup _{j \geq k} X_{j} /(j-1)$ implies that, with probability one, $\left|B_{H}(t) / t\right| \rightarrow 0$, as $t \rightarrow \infty$. The case $t \rightarrow-\infty$ is treated similarly.

Corollary 3.1. With probability one, we have $\inf _{t \in \mathbb{R}}\left(B_{H}(t)+t^{2}\right)>-\infty$.

Proof. By Lemma 3.1, with probability one, we have $B_{H}(t) / t \rightarrow 0$, as $|t| \rightarrow \infty$. Thus, with probability one, given $C>0, \exists T_{c}$ such that $\left|B_{H}(t) / t\right| \leq C, \forall t>$ $T_{c}$. Hence $\inf _{t \in\left[-T_{c}, T_{c}\right]}\left(B_{H}(t)+t^{2}\right)>-\infty$ and consequently $\left|B_{H}(t)+t^{2}\right| \geq$ $t^{2}-C t>-\infty$ for $|t|>T_{c}$. This proves the result.

Proposition 3.1. Let

$$
M^{-}:=\inf _{t \in(-\infty, 0]} \xi_{H}(t) \quad \text { and } \quad M^{+}:=\inf _{t \in[0, \infty)} \xi_{H}(t)
$$

be the values of the infima of $\xi_{H}$ over the negative and positive half-lines, respectively. We then have that:

(i) $\mathbb{P}\left\{M^{-}<0\right\}=\mathbb{P}\left\{M^{+}<0\right\}=1$.

(ii) $\mathbb{P}\left\{M^{-}=M^{+}\right\}=0$.

Proof. (i) Note that $\mathbb{P}\left\{M^{+}>0\right\}=1$ is equivalent to $\mathbb{P}\left\{B_{H}(t)+t^{2} \geq 0, \forall t>\right.$ $0\}=0$. By the self-similarity of $B_{H}$, for all $c>0$, we have that

$$
\begin{aligned}
\mathbb{P}\left\{B_{H}(t)+t^{2}>0, \forall t>0\right\} & =\mathbb{P}\left\{c^{-H} B_{H}(c t)+t^{2} \geq 0, \forall t>0\right\} \\
& =\mathbb{P}\left\{B_{H}(\tau) \geq-c^{H-2} \tau^{2}, \forall \tau>0\right\} \\
& =: \mathbb{P}\left(A_{c}\right) \equiv \text { const. }
\end{aligned}
$$

Note that $A_{c} \downarrow\left\{B_{H}(\tau) \geq 0, \forall \tau>0\right\}$ as $c \rightarrow \infty$ and since the above probability does not depend on $c>0$, it is enough to show that $\mathbb{P}\left\{B_{H}(\tau) \geq\right.$ $0, \forall \tau>0\}=0$. This follows from law of iterated logarithm for fractional Brownian motion (see Oodaira, 1972[16]).

(ii) In view of (i), part (ii) readily follows from Lemma 2.6 in Kim and Pollard (1990)[14] applied to the process $\left\{\xi_{H}(t)\right\}_{t \in \mathbb{R}}$.

Finally we state the main results of this section.

Theorem 3.1. The set where $\mathcal{T}\left(\xi_{H}\right)$ and $\mathcal{T}_{0}\left(\xi_{H}\right)$ differ is contained in a compact set almost surely. 
Proof. As $\xi_{H}$ has a continuous path almost surely by Lemma 2.2 the set where $\mathcal{T}\left(\xi_{H}\right)$ and $\mathcal{T}_{0}\left(\xi_{H}\right)$ differ is contained in the closed interval $\left[x_{*}, x^{*}\right]$, where $x_{*}$ and $x^{*}$ are as in the Lemma 2.3. It remains to prove that $x_{*}$ and $x^{*}$ are finite almost surely.

Lemma 3.1 implies that $\xi_{H}(t) / t \stackrel{\text { a.s. }}{\rightarrow} \infty$ as $t \rightarrow \infty$. Thus, Lemma 2.1 applied to the sample paths of $\xi_{H}$ yields that $x_{*}$ and $x^{*}$ are finite with probability one.

Theorem 3.2. We have $\mathbb{P}(\mathbb{T}=0)=0$ under both short- and long-range dependence.

Proof. Focus on the one-sided minorant $\mathcal{T}_{(-\infty, 0)}\left(\xi_{H}\right)(t)$ for $t \in(-\infty, 0)$. By Proposition 3.1, we have that $M^{-}=\inf _{t \in(-\infty, 0)} \xi_{H}(t)$ is almost surely negative and since $\xi_{H}(t) \rightarrow \infty$ as $t \rightarrow-\infty$, the infimum is attained at some point $t_{*}<0$. Therefore, the function $f(t)=M^{-}$is a convex minorant to $\xi_{H}(t)$ on $(-\infty, 0]$ which has touch-point at $t=t_{*}$. Thus,

$$
f\left(t_{*}\right) \leq \mathcal{T}_{(-\infty, 0)}\left(\xi_{H}\right)\left(t_{*}\right) \leq \xi_{H}\left(t_{*}\right)=f\left(t_{*}\right)
$$

and the one sided GCM $\mathcal{T}_{(-\infty, 0)}\left(\xi_{H}\right)\left(t_{*}\right)=\xi_{H}\left(t_{*}\right)$. By convexity, we have $\mathcal{L} \circ \mathcal{T}_{(-\infty, 0)}\left(\xi_{H}\right)(t) \geq 0=\mathcal{L}(f)(t)$ for all $t \in\left(t_{*}, 0\right)$. This implies that the constrained slope (defining $\mathcal{S}_{1,1}^{0}(t)$ in $\left.(1)\right)$ in $\left(t_{*}, 0\right)$ is equal to zero. One can similarly show that $\mathcal{S}_{1,1}^{0}(t)$ vanishes on an interval to the right of zero, almost surely.

By Lemma 2.3 (i), we can find an interval around 0 in which the slope of $\mathcal{T}\left(\xi_{H}\right)(t)$ is constant. By the second part of the same lemma, if this slope is zero, then we have $M^{-}=M^{+}$, where $M^{+}$and $M^{-}$are as defined in Proposition 3.1, which has probability zero by the second assertion of Proposition 3.1. This in view of (1) implies that the slope $\mathcal{S}_{1,1}(t)$ is constant and non-zero in a neighborhood of zero, almost surely.

We have thus shown that $\mathcal{S}_{1,1}^{0}(t)$ vanishes while $\mathcal{S}_{1,1}(t) \neq 0$ in a neighborhood of 0 almost surely. This by (2) implies $\mathbb{P}(\mathbb{T} \neq 0)=1$.

Lemma 3.2. With probability one, both $\mathcal{S}_{1,1}(t)$ and $\mathcal{S}_{1,1}^{0}(t)$ diverge to infinity as $t \rightarrow \infty$.

Proof. Since by Theorem 3.1, $\mathcal{S}_{1,1}(t)$ and $\mathcal{S}_{1,1}^{0}(t)$ coincide eventually as $|t| \rightarrow$ $\infty$, it is enough to prove the result for $\mathcal{S}_{1,1}(t)$.

Recall that by $(1)$, we have $\mathcal{S}_{1,1}(t)=\mathcal{L} \circ \mathcal{T}\left(\xi_{H}\right)(t)$. We shall show that for any given $C>0$, there exists $t_{0}$ s.t. $\mathcal{S}_{1,1}(t)=\mathcal{L} \circ \mathcal{T}\left(\xi_{H}\right)(t) \geq C$ for all $t>t_{0}$. To do so, we will construct a convex minorant of $\xi_{H}$, which touches $\xi_{H}$ at some point and has slope $C$ at that point. Then the GCM of $\xi_{H}$ will 
lie above this minorant and hence it will have a slope at least as large as $C$ to the right of that touch-point.

To construct such a minorant, let $c=\inf _{t \in \mathbb{R}} \xi_{H}(t)$. By Proposition 3.1 we have that $c<0$ with probability 1 . Since $\xi_{H}(t) \rightarrow \infty$ as $|t| \rightarrow \infty$, there exists a $t_{*} \in \mathbb{R}$ where the infimum of $\xi_{H}(t)$ is attained.

Consider the curve $f(t)=c+C\left(t-t_{*}\right) 1_{\left(t \geq t_{*}\right)}$. Then $f(t)$ is convex, $t_{*}$ is a common point of $\xi_{H}(t)$ and $f(t)$, and $f(t)$ is below $\xi_{H}(t)$ for $t<t_{*}$.

By Lemma 3.1 we have $\xi_{H}(t) / t \rightarrow \infty$ as $t \rightarrow \infty$. So one can find a $t^{*}>t_{*}$ such that $\xi_{H}(t)>f(t)$ for all $t>t^{*}$.

If $f(t)$ and $\xi_{H}(t)$ have no more common points in $\left(t_{*}, t^{*}\right]$, then $f(t)$ is our desired minorant. Otherwise, consider a class of functions $f_{l}(t)=c+C(t-$ $l) 1_{(t \geq l)}$. Note that if $l=t^{*}, f_{l}(t)<\xi_{H}(t)$ for all $t>t_{*}$. Define a function $d(l)=\min _{t \in\left(t_{*}, \infty\right)}\left(\xi_{H}(t)-f_{l}(t)\right)$. It is easy to see that $d$ is a continuous function of $l$. From our discussion above, $d\left(t_{*}\right) \leq 0$ and $d\left(t^{*}\right)>0$. Therefore there exists a $l_{*}$ in $\left[t_{*}, t^{*}\right)$ such that $d\left(l_{*}\right)=0$. This $f_{l_{*}}(t)$ is our desired minorant.

\section{Simulation of Quantiles of $\mathbb{L}$ and $\Psi$}

In this section we discuss simulation of quantiles of $\mathbb{L}$ and $\Psi:=-\log (\mathbb{L} / \mathbb{T}-$ 1). For simulating the quantiles of $\Psi$ and $\mathbb{L}$ we used two methods (M1) and (M2).

(M1)The method (M1) relies on approximating the limit distributions by empirical distributions for large sample sizes. To elaborate, we simulated data from a simple model $y_{i}=i / n+\epsilon_{i}$, for $i=1,2, \ldots, n$ with $n=$ $10^{6}$. The errors $\left\{\epsilon_{i}\right\}$ were therefore taken to i.i.d. normals (with variance 0.2 ) for simulating approximates to the limiting quantiles in the short range dependent case, and from fractional Gaussian noise with Hurst index $H=$ $0.7,0.8,0.9,0.95,0.99$ and variance 0.2 , for the long range dependent case. The statistics $L_{n}, T_{n}$ (not tabulated) and $\Psi_{n}$ from this simulated dataset were then calculated, and the procedure repeated $M=10^{4}$ times. The estimates of the quantiles were obtained from the sorted values of these statistics. Note that, as the limiting distributions are the same in the i.i.d. and short range cases, using the i.i.d. errors above suffices.

(M2) For simulating the quantiles of $\Psi$ and $\mathbb{L}$ by this method, we used discrete approximations of the limit processes. To this end, we simulated a sequence $Z_{j}$ of standard normal variables (for the SRD case) and fractional Gaussian noise with Hurst index $H=0.7,0.8,0.9,0.95,0.99$ (for the LRD case) of length $2 n$ where $n=10^{6}$ and constructed the partial sum process 
on $-2 \leq t \leq 2$ as follows:

$$
W_{n}(t)=\frac{1}{n^{H}}\left\{\mathbf{1}_{(t \geq 0)} \sum_{j=n+1}^{n+[n t / 2]} Z_{j}-\mathbf{1}_{(t<0)} \sum_{j=n-[n(-t) / 2]}^{n} Z_{j}\right\} .
$$

We then generated the process $Y_{n}(t)=W_{n}(t)+t^{2}$ on the grid with step size $\Delta=2 \times 10^{-6}$ and computed the greatest convex minorant $G_{m}$ and the constrained greatest convex minorant $G_{m}^{0}$ using the $\mathrm{R}$ implementation of PAVA in R package fdrtool(Klaus and Strimmer, 2013[15]). Corresponding approximations $\hat{\mathcal{S}}_{a, b}$ and $\hat{\mathcal{S}}_{a, b}^{0}$ of the slope processes $\mathcal{S}_{a, b}$ and $\mathcal{S}_{a, b}^{0}$ were obtained and numerical approximations of the random variables $\mathbb{L}$ and $\mathbb{T}$ computed using (2) where $\mathcal{S}_{a, b}$ and $\mathcal{S}_{a, b}^{0}$ were replaced by $\hat{\mathcal{S}}_{a, b}$ and $\hat{\mathcal{S}}_{a, b}^{0}$. Quantiles of the resulting statistic $\Psi=-\log (\mathbb{L} / \mathbb{T}-1)$ were calculated based on $M=10^{4}$ independent replications.

The standard errors of these quantiles are based on the observations that for large $n, F_{n}$, the distribution function of the statistic $L_{n}\left(\Psi_{n}\right.$, respectively) will be a good approximation to the corresponding limit distribution, and furthermore, if $F_{n, M}$ is the corresponding empirical CDF, based on $M$ independent Monte-Carlo samples, then

$$
\sqrt{M}\left(F_{n, M}^{\leftarrow}(\alpha)-F_{n}^{\leftarrow}(\alpha)\right) \approx_{d} N\left(0, \frac{\alpha(1-\alpha)}{f_{n}\left(F_{n}^{\leftarrow}(1-\alpha)\right)}\right)
$$

as $M \rightarrow \infty$. Here, $F_{n}^{\leftarrow}$ denotes the left continuous inverse of $F_{n}$ and $f_{n}$ denotes the density of $F_{n}$, which is assumed to exist at the point $F_{n}^{\leftarrow}(1-\alpha)$ The quality of the approximation is confirmed by the fact that M1 and M2 provide similar quantile estimates relative to the Monte-Carlo estimation error. The denominator of the variance in (5) was estimated by $\widehat{f}_{n}\left(F_{n, M}^{\leftarrow}(1-\right.$ $\alpha)$ ), where $\widehat{f}_{n}$ is obtained by differencing $F_{n, M}$.

Quantiles of $\mathbb{L}$ and $\Psi$ calculated using both the methods are presented in the Tables $1-4$. Figure 1 shows the quantiles of $\Psi$ as a function of $h$.

Remark 4.1. Based on the simulated quantiles of $\Psi$, we make the following conjectures about its distribution function, $F_{H}$, for values of the Hurst index $H$ between 0.5 (short-range dependence case) and 1.

$C 1$ For every $0<p<1, F_{H}^{-1}(p) \rightarrow \infty$ as $H \rightarrow 1$ though not monotonically.

C2 For a fixed $p, H \mapsto F_{H}^{-1}(p)$ increases at first with $H$ followed by a decrease and increase again. 
Table 1: Simulated Quantiles of $\Psi$ : Method 1

\begin{tabular}{|c|c|c|c|c|c|}
\hline $\mathrm{p}$ & $\mathrm{SRD}$ & $\mathrm{H}=0.7$ & $\mathrm{H}=0.8$ & $\mathrm{H}=0.9$ & $\mathrm{H}=0.95$ \\
\hline .10 & $-0.35(0.011)$ & $-0.02(0.011)$ & $0.17(0.015)$ & $0.33(0.052)$ & $0.58(0.062)$ \\
\hline .15 & $0.10(0.024)$ & $0.26(0.019)$ & $0.47(0.040)$ & $0.63(0.001)$ & $0.90(0.002)$ \\
\hline .20 & $0.39(0.013)$ & $0.51(0.018)$ & $0.71(0.015)$ & $0.86(0.012)$ & $1.14(0.014)$ \\
\hline .25 & $0.55(0.009)$ & $0.73(0.023)$ & $0.92(0.002)$ & $1.09(0.008)$ & $1.36(0.008)$ \\
\hline .30 & $0.83(0.027)$ & $0.96(0.026)$ & $1.13(0.036)$ & $1.29(0.020)$ & $1.58(0.030)$ \\
\hline .35 & $1.04(0.012)$ & $1.20(0.011)$ & $1.36(0.022)$ & $1.50(0.012)$ & $1.99(0.012)$ \\
\hline .40 & $1.25(0.020)$ & $1.48(0.027)$ & $1.59(0.019)$ & $1.71(0.008)$ & $2.21(0.011)$ \\
\hline .45 & $1.69(0.053)$ & $1.79(0.016)$ & $1.85(0.003)$ & $1.94(0.003)$ & $2.41(0.005)$ \\
\hline .50 & $2.21(0.021)$ & $2.19(0.006)$ & $2.11(0.012)$ & $2.20(0.051)$ & $2.66(0.025)$ \\
\hline .55 & $2.88(0.047)$ & $2.69(0.053)$ & $2.43(0.010)$ & $2.49(0.041)$ & $2.93(0.042)$ \\
\hline .60 & $3.87(0.068)$ & $3.41(0.030)$ & $2.88(0.028)$ & $2.80(0.015)$ & $3.24(0.020)$ \\
\hline .65 & $6.28(0.103)$ & $4.69(0.310)$ & $3.47(0.067)$ & $3.18(0.038)$ & $3.59(0.072)$ \\
\hline .70 & $20.03(0.022)$ & $7.92(0.349)$ & $4.31(0.173)$ & $3.70(0.011)$ & $4.03(0.097)$ \\
\hline .75 & $23.91(0.032)$ & $22.82(0.060)$ & $5.95(0.286)$ & $4.40(0.035)$ & $4.68(0.060)$ \\
\hline .80 & $24.25(0.020)$ & $23.79(0.019)$ & $10.89(0.494)$ & $5.72(0.132)$ & $5.77(0.122)$ \\
\hline .85 & $24.67(0.022)$ & $24.51(0.036)$ & $24.14(0.023)$ & $8.43(0.539)$ & $8.30(0.096)$ \\
\hline .90 & $25.00(0.041)$ & $25.12(0.031)$ & $25.28(0.054)$ & $26.43(0.165)$ & $27.05(0.248)$ \\
\hline .95 & $25.21(0.023)$ & $25.92(0.017)$ & $26.32(0.026)$ & $28.02(0.489)$ & $33.13(0.188)$ \\
\hline
\end{tabular}

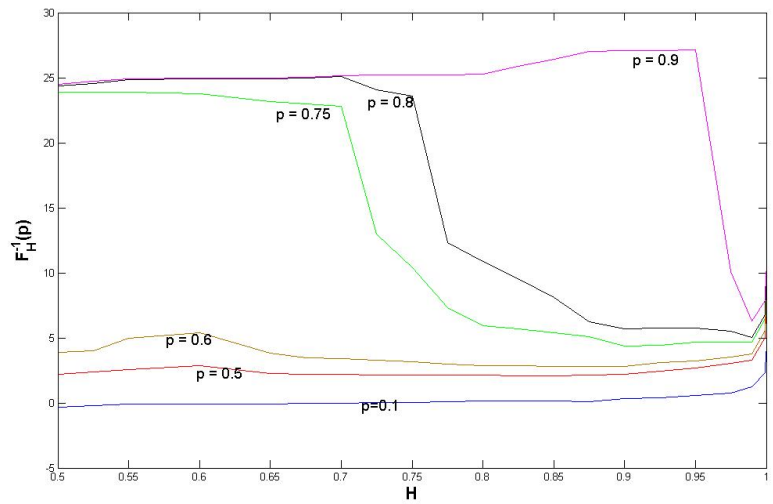

Figure 1: Quantiles of $\Psi$ as a function of $H$ 
Table 2: Simulated Quantiles of $\Psi$ : Method 2

\begin{tabular}{|c|c|c|c|c|c|}
\hline $\mathrm{p}$ & $\mathrm{SRD}$ & $\mathrm{H}=0.7$ & $\mathrm{H}=0.8$ & $\mathrm{H}=0.9$ & $\mathrm{H}=0.95$ \\
\hline .10 & $-0.34(0.011)$ & $-0.02(0.011)$ & $0.15(0.015)$ & $0.33(0.052)$ & $0.59(0.062)$ \\
\hline .15 & $0.09(0.024)$ & $0.24(0.019)$ & $0.44(0.040)$ & $0.63(0.001)$ & $0.91(0.002)$ \\
\hline .20 & $0.38(0.013)$ & $0.50(0.018)$ & $0.69(0.015)$ & $0.85(0.012)$ & $1.13(0.014)$ \\
\hline .25 & $0.54(0.009)$ & $0.77(0.023)$ & $0.93(0.002)$ & $1.10(0.008)$ & $1.36(0.008)$ \\
\hline .30 & $0.85(0.027)$ & $0.91(0.026)$ & $1.11(0.036)$ & $1.30(0.020)$ & $1.58(0.030)$ \\
\hline .35 & $1.02(0.012)$ & $1.19(0.011)$ & $1.39(0.022)$ & $1.52(0.012)$ & $1.99(0.012)$ \\
\hline .40 & $1.25(0.020)$ & $1.44(0.027)$ & $1.60(0.019)$ & $1.72(0.008)$ & $2.22(0.011)$ \\
\hline .45 & $1.70(0.053)$ & $1.78(0.016)$ & $1.84(0.003)$ & $1.95(0.003)$ & $2.40(0.005)$ \\
\hline .50 & $2.23(0.021)$ & $2.17(0.006)$ & $2.10(0.012)$ & $2.21(0.051)$ & $2.70(0.025)$ \\
\hline .55 & $2.85(0.047)$ & $2.70(0.053)$ & $2.45(0.010)$ & $2.51(0.041)$ & $2.92(0.042)$ \\
\hline .60 & $3.90(0.068)$ & $3.38(0.030)$ & $2.85(0.028)$ & $2.83(0.015)$ & $3.27(0.020)$ \\
\hline .65 & $6.30(0.103)$ & $4.48(0.310)$ & $3.50(0.067)$ & $3.21(0.038)$ & $3.59(0.072)$ \\
\hline .70 & $20.02(0.022)$ & $7.80(0.349)$ & $4.20(0.173)$ & $3.71(0.011)$ & $4.04(0.097)$ \\
\hline .75 & $23.89(0.032)$ & $22.80(0.060)$ & $6.12(0.286)$ & $4.40(0.035)$ & $4.66(0.060)$ \\
\hline .80 & $24.22(0.020)$ & $23.76(0.019)$ & $10.80(0.494)$ & $5.83(0.132)$ & $5.73(0.122)$ \\
\hline .85 & $24.61(0.022)$ & $24.49(0.036)$ & $24.10(0.023)$ & $8.51(0.539)$ & $8.24(0.096)$ \\
\hline .90 & $25.01(0.041)$ & $25.16(0.031)$ & $25.29(0.054)$ & $26.51(0.165)$ & $27.10(0.248)$ \\
\hline .95 & $25.27(0.023)$ & $25.90(0.017)$ & $26.30(0.026)$ & $28.13(0.489)$ & $33.15(0.188)$ \\
\hline
\end{tabular}


Table 3: Simulated Quantiles of $\mathbb{L}$ : Method 1

\begin{tabular}{|c|c|c|c|c|c|}
\hline $\mathrm{p}$ & $\mathrm{SRD}$ & $\mathrm{H}=0.7$ & $\mathrm{H}=0.8$ & $\mathrm{H}=0.9$ & $\mathrm{H}=0.95$ \\
\hline .10 & $0.01(0.001)$ & $0.02(0.001)$ & $0.03(0.000)$ & $0.04(0.000)$ & $0.05(0.000)$ \\
\hline .15 & $0.02(0.000)$ & $0.05(0.000)$ & $0.08(0.004)$ & $0.09(0.005)$ & $0.10(0.004)$ \\
\hline .20 & $0.04(0.001)$ & $0.10(0.000)$ & $0.14(0.018)$ & $0.18(0.018)$ & $0.19(0.018)$ \\
\hline .25 & $0.06(0.001)$ & $0.16(0.000)$ & $0.23(0.003)$ & $0.29(0.004)$ & $0.31(0.004)$ \\
\hline .30 & $0.10(0.002)$ & $0.24(0.001)$ & $0.34(0.002)$ & $0.36(0.002)$ & $0.39(0.003)$ \\
\hline .35 & $0.13(0.004)$ & $0.34(0.010)$ & $0.48(0.028)$ & $0.50(0.029)$ & $0.51(0.025)$ \\
\hline .40 & $0.17(0.005)$ & $0.44(0.018)$ & $0.66(0.005)$ & $0.69(0.005)$ & $0.72(0.005)$ \\
\hline .45 & $0.22(0.010)$ & $0.57(0.012)$ & $0.87(0.011)$ & $0.90(0.011)$ & $0.92(0.011)$ \\
\hline .50 & $0.28(0.009)$ & $0.71(0.010)$ & $1.12(0.039)$ & $1.47(0.041)$ & $1.61(0.033)$ \\
\hline .55 & $0.35(0.013)$ & $0.91(0.042)$ & $1.43(0.034)$ & $1.61(0.036)$ & $1.75(0.035)$ \\
\hline .60 & $0.43(0.020)$ & $1.14(0.068)$ & $1.79(0.028)$ & $1.88(0.029)$ & $1.92(0.031)$ \\
\hline .65 & $0.54(0.005)$ & $1.42(0.009)$ & $2.21(0.024)$ & $2.49(0.026)$ & $2.62(0.027)$ \\
\hline .70 & $0.66(0.002)$ & $1.76(0.037)$ & $2.79(0.041)$ & $3.25(0.043)$ & $3.87(0.042)$ \\
\hline .75 & $0.82(0.004)$ & $2.18(0.000)$ & $3.52(0.019)$ & $4.37(0.020)$ & $4.99(0.020)$ \\
\hline .80 & $1.00(0.002)$ & $2.77(0.017)$ & $4.48(0.044)$ & $5.01(0.047)$ & $5.36(0.045)$ \\
\hline .85 & $1.23(0.003)$ & $3.56(0.045)$ & $5.85(0.018)$ & $6.66(0.019)$ & $7.11(0.018)$ \\
\hline .90 & $1.62(0.002)$ & $4.63(0.035)$ & $7.74(0.060)$ & $9.64(0.063)$ & $10.21(0.063)$ \\
\hline .95 & $2.26(0.006)$ & $6.64(0.120)$ & $11.23(0.029)$ & $15.61(0.031)$ & $19.91(0.033)$ \\
\hline
\end{tabular}


Table 4: Simulated Quantiles of $\mathbb{L}:$ Method 2

\begin{tabular}{|c|c|c|c|c|c|}
\hline $\mathrm{p}$ & $\mathrm{SRD}$ & $\mathrm{H}=0.7$ & $\mathrm{H}=0.8$ & $\mathrm{H}=0.9$ & $\mathrm{H}=0.95$ \\
\hline .10 & $0.01(0.001)$ & $0.03(0.001)$ & $0.03(0.000)$ & $0.04(0.000)$ & $0.05(0.000)$ \\
\hline .15 & $0.02(0.000)$ & $0.05(0.000)$ & $0.08(0.004)$ & $0.08(0.005)$ & $0.10(0.004)$ \\
\hline .20 & $0.04(0.001)$ & $0.10(0.000)$ & $0.15(0.018)$ & $0.18(0.018)$ & $0.19(0.018)$ \\
\hline .25 & $0.07(0.001)$ & $0.16(0.000)$ & $0.23(0.003)$ & $0.29(0.004)$ & $0.31(0.004)$ \\
\hline .30 & $0.09(0.002)$ & $0.24(0.001)$ & $0.34(0.002)$ & $0.36(0.002)$ & $0.39(0.003)$ \\
\hline .35 & $0.13(0.004)$ & $0.35(0.010)$ & $0.50(0.028)$ & $0.48(0.029)$ & $0.52(0.025)$ \\
\hline .40 & $0.17(0.005)$ & $0.43(0.018)$ & $0.66(0.005)$ & $0.69(0.005)$ & $0.71(0.005)$ \\
\hline .45 & $0.24(0.010)$ & $0.57(0.012)$ & $0.85(0.011)$ & $0.90(0.011)$ & $0.93(0.011)$ \\
\hline .50 & $0.28(0.009)$ & $0.71(0.010)$ & $1.10(0.039)$ & $1.48(0.041)$ & $1.61(0.033)$ \\
\hline .55 & $0.35(0.013)$ & $0.97(0.042)$ & $1.39(0.034)$ & $1.62(0.036)$ & $1.76(0.035)$ \\
\hline .60 & $0.44(0.020)$ & $1.13(0.068)$ & $1.76(0.028)$ & $1.90(0.029)$ & $1.92(0.031)$ \\
\hline .65 & $0.55(0.005)$ & $1.42(0.009)$ & $2.25(0.024)$ & $2.51(0.026)$ & $2.61(0.027)$ \\
\hline .70 & $0.65(0.002)$ & $1.78(0.037)$ & $2.82(0.041)$ & $3.28(0.043)$ & $3.87(0.042)$ \\
\hline .75 & $0.80(0.004)$ & $2.18(0.000)$ & $3.51(0.019)$ & $4.38(0.020)$ & $5.01(0.020)$ \\
\hline .80 & $1.00(0.002)$ & $2.74(0.017)$ & $4.42(0.044)$ & $5.06(0.047)$ & $5.36(0.045)$ \\
\hline .85 & $1.23(0.003)$ & $3.52(0.045)$ & $5.84(0.018)$ & $6.67(0.019)$ & $7.13(0.018)$ \\
\hline .90 & $1.62(0.002)$ & $4.67(0.035)$ & $7.79(0.060)$ & $9.66(0.063)$ & $10.24(0.063)$ \\
\hline .95 & $2.25(0.006)$ & $6.68(0.120)$ & $11.19(0.029)$ & $15.63(0.031)$ & $20.00(0.033)$ \\
\hline
\end{tabular}


These observations, in particular, the presence of partial nesting, are important since they provide a way of avoiding the estimation of the Hurst parameter $H$ while constructing C.I.s in certain situations.

Remark 4.2. In the boundary case $H=1$, the fractional Brownian motion is degenerate and equals $\mathbb{B}_{H}(t)=Z t, t \in \mathbb{R}$, for some centered Normal random variable $Z$ (recall (11)). So the sample paths of $\mathbb{G}_{a, b}(t)=a \mathbb{B}_{H}(t)+b t^{2}$ are convex and therefore coincide with their GCM. Similarly the one sided GCMs also coincide with the original curve. With this explicit characterization of $G C M$ by (2) we get $\mathbb{L}_{a, b}=\mathbb{T}_{a, b}=\int_{D_{0}} \mathcal{S}_{a, b}(z)^{2} d z$, where $D_{0}=\{z$ : $\left.\mathcal{S}_{a, b}^{0}(z)=0\right\}$. This gives $\mathcal{R}=1$ and $\Psi=\infty$ with probability 1, leading us to conjecture that the distribution of $\Psi=\Psi_{H}$ converges to the degenrate distribution at $\infty$, as $H \uparrow 1$. This, however, appears hard to verify numerically since when $H$ very close to 1 , the simulation methods for fBm break down.

\section{References}

[1] Adler, R. J. and Taylor, J. E.(2005) Random Fields and Geometry. Springer, New York.

[2] Anevski, D. and Hossjer, O.(2006) A general asymptotic scheme for inference under order restrictions. The Annals of Statistics Vol. 34, 18741930.

[3] Banerjee, M. and Wellner, J. A.(2001) Likelihood ratio tests for monotone functions. The Annals of Statistics Vol. 29, 1699-1731.

[4] Banerjee, M. and Wellner, J. A.(2005) Confidence intervals for current status data. Scandinavian Journal of Statistics Vol. 32 405-424.

[5] Banerjee, M. (2009) Inference in exponential family regression models under certain shape constraints. Advances in Multivariate Statistical Methods, Statistical Science and Interdisciplinary Research, Vol. 4, 249-272. World Scientific.

[6] Brunk, H. D. (1970) Estimation of isotonic regression. In Nonparametric Techniques in Statistical Inference (M. L. Puri, ed,) 177-197. Cambridge University Press, London.

[7] Dehling, H., and Taqqu, M. S. (1989). The empirical process of some long-range dependent sequences with an application to U-statistics. The Annals of Statistics, 1767-1783. 
[8] Groeneboom, P. (1985). Estimating a monotone density. Proceeding of the Berkeley Conference in Honor of Jezry Neyman and Jack Kiefer Vol II, (Lucien, M. LeCam and Richard A. Olshen eds.)

[9] Groeneboom, P. (1989). Brownian motion with a parabolic drift and airy function. Probability Theory and Related Fields Vol 81, 79-109.

[10] Groeneboom, P. (2011). Vertices of the least concave majorant of Brownian motion with parabolic drift. Electronic Journal of Probability vol. 16, 2234-2258.

[11] Groeneboom, P., Jongbloed, G. and Witte, B. I. (2010). Maximum smoothed likelihood estimation and smoothed maximum likelihood estimation in the current status model. The Annals of Statistics, Vol. 38, $352-387$.

[12] Groeneboom, P. and Wellner J.A. (1992). Information Bounds and Nonparametric Likelihood Estimation. Birkhauser, Basel.

[13] Groeneboom, P. and Wellner J.A. (2001). Computing Chernoff's distribution. Journal of Computational and Graphical Statistics. Vol. 10, 388-400.

[14] Kim, J. and Pollard, D. (1990). Cube Root Asymptotics. The Annals of Statistics Vol 18 191-219.

[15] Klaus, B. and Strimmer, K. (2013). Package "fdrtool" http://cran. r-project.org/web/packages/fdrtool/fdrtool.pdf.

[16] Oodaira, H. (1972). On Strassens's version of the law of the iterated logarithm for Gaussian processes. Z. Wahrscheinlichkeitstheorie verw. Geb. 21, 289-299.

[17] Zhao, O. and Woodroofe, M.(2012) Estimating a monotone trend. Statistica Sinica, Vol. 22, 359-378. 